Comparing the impact of management on public and private nurses in Bangladesh

\begin{tabular}{|r|l|}
\hline Journal: & International Journal of Public Sector Management \\
\hline Manuscript ID & IJPSM-01-2019-0023.R2 \\
\hline Manuscript Type: & Original Article \\
\hline Keywords: & $\begin{array}{l}\text { Public Sector Management, Private sector organizations, Organizational } \\
\text { behaviour }\end{array}$ \\
\hline \multicolumn{2}{|l}{} \\
\hline
\end{tabular}

SCHOLARONE ${ }^{\text {m }}$

Manuscripts 


\title{
Comparing the impact of management on public and private nurses in Bangladesh
}

\begin{abstract}
Purpose: This paper uses theory as a lens for comparing the impact of management on Bangladeshi public and private nurses' perception of work harassment, wellbeing and turnover intentions where Anglo-American management models have been super-imposed on an existing different culture.
\end{abstract}

Design: Survey data was collected from 317 Bangladeshi nurses' (131 from the public sector and 186 from the private sector). Structural Equation Modelling was used for analysis.

Findings: Good management was associated with high wellbeing, and high work harassment was associated with low wellbeing. However, management explained approximately a quarter of private sector nurse's wellbeing, and work harassment had no impact on this association. In total, management, work harassment and wellbeing explained approximately a third of the turnover intentions of public sector nurses, whereas only work harassment explained approximately a third of private sector nurses' turnover intentions.

Limitation; Cross-sectional data is susceptible to common method bias. A common latent factor was included, and several items that were explained by common method variance were controlled. Further, the findings are limited by the sample size from one sector and the use of only one developing country.

Implications: It is a waste of resources to transplant Anglo-American management models to developing countries without understanding the cultural context.

Originality: Anglo-American management models are not easily transferable to the public sector Bangladesh context probably because of the impact of ties and corruption. Management is a positive resource that can build wellbeing for public sector employees only. 
Keywords: public-private sector, management, well-being, work harassment, turnover intentions 


\section{Comparing the impact of management on public and private nurses in Bangladesh}

Public sector management models do not easily translate to best practice in developing countries, mainly because the conditions are too different. For example, in contrast to developed countries, there are more doctors than nurses in Bangladesh and the demand for their services exceeds supply whilst continuing to grow in line with population growth: hence they work in high-pressure environments (World Health Organization, 2018; World Bank, 2016). As a consequence, this paper examines whether Bangladeshi nurses perceive work harassment similarly to other public and private nurses in developed countries (Brunetto, Xerri, Trinchero, Farr-Wharton, Shacklock, Borgonovi, 2016) and whether this affects their turnover intention. Dick (2010) refers to work harassment as a form of workplace bullying but suggests that it differs from bullying. Specifically, an employee is exposed to work harassment when their employing organisation unreasonably monitors their actions, sets excessively high workloads, and has stringent accountability standards (Brunetto et al, 2016b). As such, the organisation and its managers are the instigators of work harassment.

Work harassment has been identified as a growing problem for public sector managers in developed countries (Tummers, Brunetto \& Teo 2016). For example, Brunetto et al. (2016b) compared work harassment of nurses in Australia and Italy and found significant parallels in their perceptions. More recent research identified a growing presence and acceptance of work harassment in US and UK hospitals (Farr-Wharton, Brunetto \& Xerri, 2019). The delivery of health services has been significantly affected by the cost-constraint that mandates the depth and breadth of reforms over the past four decades which has resulted in the expectation that employees will 'do more with less' (Diefenbach, 2009). However, the situation is even more pronounced in Bangladesh, where the shortages in nurses, especially in the public sector and in rural areas is a growing problem (Ahmed \& Shirahada, 2017). Specifically, there are high levels of resource inadequacy, with widespread shortages in basic nursing equipment and a 
lack of an effective nurse management systems (World Health Organization, 2016). Such inadequacies in the Bangladesh system are likely to create a potential haven for work harassment.

The shortage of public sector nurses in Bangladesh is increasingly dire, and in some cases, the families of patients have to act as carers (Mahdy, 2009). If work harassment is not managed effectively, then it may negatively influence the profession's ability to attract new nurses, and retain them in the job. Whilst there is evidence of work harassment across different public sector occupations, such as local government and healthcare workers across different countries - Australia, Italy, USA (Tummers et al 2016; Xerri et al, 2016), there is no evidence as to whether work harassment is an issue in developing countries. In this study, we have two core aims: firstly, to develop insight into the impact of management on outcomes such as work harassment, wellbeing and turnover intentions for nurses working in Bangladeshi hospitals, and secondly, to develop a deeper understanding of the similarities and differences across the public and private sectors.

In relation to work harassment, in developed countries the theory argues that the role of the manager for professionals is very important because of the dual role of the professional mentor and line manager within the organisational hierarchy. However, the managers of professionals are also increasingly faced with the issue of 'professional hybridity' (the sometimes-contradictory expectations that managers will both develop nurses' professional skills and knowledge and ensure task completion) (Noordegraaf, 2015). Never-the-less, a good manager is one who can mediate the excessive demands of the hierarchy to ensure appropriate resourcing and support is available in relation to task completion and professional development (Farr-Wharton, Brunetto, \& Shacklock, 2011). Although past evidence has established the association between management practices and wellbeing, and between management practices and turnover intentions (Brunetto et al., 2013), to the best knowledge of the authors, there is 
no evidence about how work harassment may change these established relationships. As such, we aim to add to the existing evidence by examining the role of work harassment in affecting the association between satisfaction with management and employee wellbeing, and in turn, turnover intentions within a developing country such as Bangladesh. Such evidence adds to the literature about the impact of management on employee wellbeing and turnover intentions of nurses within developing countries, in this case, Bangladesh.

Managing both public and private sector employees in developing countries can be challenging as international HR systems are imported into existing culture-driven decisionmaking systems. Most managers were traditionally autocratic in their practices in South Asia (Khan, Miah \& Manzoor, 2014). However, in the last three decades, the service sector has undergone significant change mainly because of global forces and government reforms that have elevated the importance of human resources slowly, especially in terms of the significance of ensuring adequate qualifications and competencies for key positions. This change has led to the introduction of modern HRM and strategic systems, however, the new systems have not replaced the previous stronghold of culture in organisational decision-making. This means that family ties and corruption still dominate decision making, favouring some and disadvantaging the majority, and whilst those with strong international connections are likely to have basic HR policies and structures evident in countries such as the USA and UK, the difference is in their ability to implement those policies into practice (Khan et al., 2014, p 99). Hence, while Bangladeshi organisations may have specific policies about work hours and duties, in practice, there is probably greater scope for work harassment in Bangladesh compared with AngloAmerican countries such as the UK, USA and Australia.

This paper compares the relationship between management, work harassment, wellbeing and turnover intentions of public and private sector nurses using a Conservation of Resources (COR) theoretical framework (Hobfoll, 1989). COR explains employee outcomes 
regarding their ability to maintain and grow their personal resources such as health and wellbeing. COR theorists argue that when the organisational environment erodes their personal resources through work harassment, their wellbeing is negatively impacted and this motivates them to protect their well-being by regulating their work practices. COR theorists argue that if extra support (e.g., from managers) is not available, then employees may offset perceived resource losses, and potential shortages, by reducing effort at work or leaving the organisation (Hobfoll, 2011). The research questions (RQs) guiding the study are:

$R Q$ 1: What is the impact of management on work harassment, wellbeing and turnover of public and private sector Bangladeshi nurses?

$R Q$ 2: What are similarities and differences in the relationship between management, work harassment, wellbeing and turnover for nurses in the public and private sector?

The next part details a relevant review of the literature and identifies hypotheses that reflect the gap in the literature.

\section{Background}

\section{Conversation of Resources (COR) theory}

The COR theory is ideal for understanding employee outcomes, especially in the face of resource adversity (Hobfoll 1989). The way COR theory works is via two principles and four Corollaries (Hobfoll, 2011). Principle one: a loss of resources likely has a bigger effect than acquiring new resources. Principle two: to gain new or more resources, employees must firstly spend time or energy (resources) in those activities likely to lead to more resources. Further, if employees believe they are likely to experience a loss of resources (such as a loss of wellbeing) they will likely engage in activities (sick leave/ look for another job) to protect themselves against falling wellbeing. The four corollaries include: (1) the higher employees' perception of resources, the easier it is for them to gain resources, whereas employees with fewer resources are more susceptible to resource loss, and less able to gain resources. (2) It is 
likely that an initial loss of resources will lead to further losses; whereas (3) it is likely that a resource gain will likely lead to further resources gains; and (4) employees are likely to act defensively as a strategy to prevent further loss of resources (Halbesleben, Neveu, PaustianUnderdahl, \& Westman, 2014).

Drawing from COR theory, each organisation makes decisions that produce either positive or negative environmental conditions, called 'resource caravan passageways' (Hobfoll (2011). Under ideal conditions, an employee can build resources such as increased wellbeing by investing other resources at their disposal; in this case, effective supervision is a social resource (caravan passageway) an employee can draw upon. Whereas, if the resource caravan passageway is high levels of work harassment, employees have low resources and are thus less able to orchestrate resource gain. In this case, we propose that work harassment may result in employees that are less able to develop wellbeing with the social resource derived from the relationship with their manager.

Using COR theory to explain the relationship between management, work harassment, wellbeing and turnover, we argue that effective management practices is a positive resource caravan passageway that can build wellbeing, whereas work harassment is a negative resource caravan passageway that will influence an employee's ability to develop resources such as wellbeing. The consequences of the poor environmental conditions are likely to lead to increased turnover (Hobfoll, 2001). Using COR corollary 4, the issue will be whether the support from the manager is enough of a 'cushion' to form a barrier against the negative influence of work harassment on wellbeing, and the flow on to an intention to turnover. As per corollary 4 , those with a lack of resources are likely to act defensively to conserve against further resource loss. That is, those who lack resources are unable to apply resources to fight against resource loss (Hobfoll, 2011), and thus to conserve further loss, they may choose to eject themselves from the situation by intending to leave their employing organisation. In 
contrast, those employee with adequate resources are likely to be able to invest them in resource develop, which is likely to be evident by high wellbeing and low turnover intentions.

\section{Variables under examination}

\section{Work Harassment (resource caravan passageway)}

The term 'work harassment' is challenged by different groups of researchers and the same work has multiple meanings depending on the country. Originally, the term emerged from 'harassment' meaning “... all repeated behaviors [sic] which ... provoke, frighten, intimidate, or bring discomfort to the victim" (Brodsky (1976) cited in Einarsen, 2000; p382). However, the definition has moved on in different ways in different countries. For example, the Scandinavian researchers adopted different terms compared with the Anglo-American researchers for similar behavioural aggression and their remains no consensus about definitions for the different negative work behaviours (Branch, Ramsay, \& Barker, 2013; Einarsen, \& Nielsen, 2015). In terms of public sector HR in Anglo-American countries, work harassment has emerged as a negative work behaviour, similar to bullying or mobbing because it is characterised by repeated ill-treatment with negative consequences for the individual (Zapf, Escartin, \& Einarsen, 2011).

Originally, work harassment was conceptualised as part of bullying because of its negative impact on the individual (Raynor, 2002). More recently, work harassment is differentiated from bullying for three main reasons. Firstly, bullying is directed at an individual, whereas work harassment is about task completion and affects large numbers of front-line employees facing a chronic problem of demand outstripping supply, forcing them either to choose 'who get what' (or who misses out) and/or 'to do more with less' on a continual basis (Dick, 2010; Xerri, Farr-Wharton, Brunetto, \& Lambries, 2016). Secondly, bullying can involve physical or mental abuse directed at an individual, whereas work harassment is not 
personal. Instead, it is the result of a resourcing decision made originally by governments, and then by senior management in allocating resources to each department (Tummers, et al, 2016).

Thirdly, the incidence of work harassment relies on the power of professional principles to ensure patients/clients/customers are serviced, irrespective of organisational resourcing constraints. This is because universities and the professional bodies regulate entry into the professions and work practices are underpinned by specific norms and values (such as professional standards of service and effectiveness) (Farr-Wharton et al., 2011) that often brings them into conflict with organisational norms (task completion/ reduced per capita costs) leading to 'professional hybridity' (Noordegraaf, 2015). One of the outcomes for healthcare professionals is increasing stress from role conflict as professionals work overtime (often against organisational directions) (Tummers, Vermeeren, Steijn, \& Bekkers, 2012). Hence, work harassment is a type of negative workplace behaviour that is emerging as a growing HR problem, especially for healthcare professionals working in the public sector where austerity dominates resourcing decisions. In particular, Tummers et al. (2016) identified the rise in stress (and stress-related illnesses) emerging amongst professionals because of increasing work harassment, role overload and role ambiguity.

For healthcare workers, high demand is a worldwide phenomenon. In those countries where there is also a shortage of nurses, the workplace conditions are even more likely to enhance, rather than detract from work harassment. For example, Laschinger and Fida (2014) found that in Canada, even new graduate nurses were reporting high-stress levels leading to falling wellbeing and the worst cases, a steady rise in the incidence of high levels of burnout and turnover. Laschinger et al. (2014, p19) argue that the key contributors are heavy workloads and inadequate staffing that in turn, create the negative environmental conditions ripe for increasing job dissatisfaction, and: 
"Similar to other helping professions, the prevalence of burnout in nursing is particularly high, because of the high emotional and physical demands of this work"

However, it may be the impact of cultural factors intervening for Bangladeshi nurses that negates the impact of work harassment if for example, they perceive the importance of helping pateints are over-riding the impact of high workloads on their wellbeing. This is what is unknown - do Bangladeshi employees such as nurses behave similarly to employees in developed countries? In terms of COR theory, high work harassment acts as a negative work behaviour that erodes personal resources. If an employee has a shortage of resources - they will likely see a reduction in their wellbeing. As such, principle two is enacted and consequently, employees seek to control the negative flow of wellbeing by looking for another job (as one example of acting defensively) (Hobfoll 2011). Unless there is a counterbalance of positive resources, for example - positive relationships with their managers that can buffer and negate the impact of work harassment, then employees' personal resources will be negatively affected.

\section{Employee Resources (resource caravans)}

\section{Manager Support - Leader-member exchange (a social support resource)}

Manager-subordinate relationships can be conceptualised via the Leader-Member Exchange (LMX) framework. LMX is a Social exchange theory (SET) variable because the potential benefits from effective workplace relationships far outweigh the initial outlay of good will. LMX argues that managers form different quality relationships with employees: for some their workplace interactions are meaningful and over time leads to mutual reciprocity (insiders), whereas for others the interactions are negative and consequently a relationship forms that is built only on the work contract specifications (Martin, Thomas, Guillaume, Lee, \& Epitropaki, 2016). The benefits of an effective LMX relationship were identified in a meta- 
analysis by Martin et al. (2016) as high role performance and high citizenship behaviour as well as low counteractive workplace behaviour. This relationship becomes even more important when it involves professionals because there is an added responsibility on the part of the manager to ensure the employee is socialised into the values, norms and practices associated with the profession and adheres to professional standards (Brunetto et al., 2018). However, research about public sector nurses in Brazil found that that the theories and "truths" accepted as the behaviour of employees such as nurses in developed countries does not always translate to the behaviour of nurses in BRIC countries. For example, Brunetto, Shacklock, Teo, Farr-Wharton \& Nelson (2015) found that managers had minimal impact on Brazilian nurses’ behaviour for numerous reasons, including that there were different contracts in place with some nurses reporting to managers in the capital of Brazil, not the hospital, and the impact of corruption on nurses' actions in the workplace.

However, using COR theory, when LMX is high, it suggests that employees can access support from managers or someone in management. Hence, it is not surprising that previous research in developed countries suggests that high LMX is associated with high wellbeing and low turnover for nurses in Anglo-American countries such as Australia, Italy and the UK (Brunetto et al., 2018) as well as the USA (Brunetto et al., 2013). The reason as to why LMX is associated with high employee performance is because as Lashinger and Fida (2014) found in their research of Canadian nurses, high LMX indicates that nurses are receiving high levels of support from managers, which in turn provides a barrier to stress and helps maintain high employee wellbeing. However, the relationship has not been examined for public and private sector Bangladeshi nurses, where cultural influences on HR and workplace relationships do not necessarily follow the Anglo-Celtic model. To test whether similar relationships are in play with Bangladesh for nurses, the following hypotheses are used.

\section{H1. LMX is negatively related to turnover intention}




\section{H2. LMX is positively related to employee wellbeing}

Employee well-being (an individual/personal resource)

'Well-being' is a general term with multiple definitions across different disciplines and there is no agreement on a definition except; however, that it is a multi-faceted concept (incorporating physical, job-related, psychological, social wellbeing), and each time it is used, it requires explicit defining in order to avoid confusion (Seligman, 2012). Employee well-being is defined as a composite state that encompasses a hedonic component (life satisfaction) and a eudaimonic component (living life according to one's values) (Forgeard, Jayawickreme, Kern, \& Seligman, 2011) within an occupational context (van der Doef and Maes 1999). Past research argues that high workloads erode nurses' well-being because wellbeing is the barometer indicating how well they are coping (Brunetto et al., 2017). Previous research shows that high wellbeing predicts low turnover for public sector Australian nurses (Brunetto, Rodwell, Shacklock, Farr-Wharton, \& Demir, 2016a) and high work harassment is associated with low wellbeing for Australian and Italian public and private sector nurses (Brunetto et al., 2016b).

\section{Turnover Intentions}

Turnover intentions refers to employees' propensity to withdraw from their present workplace and consequently, they conserve their energy at work and redirect it towards looking at alternatives (Van Knippenberg, Van Dick, \& And Tavares, 2007). There are multiple push factors likely to promote turnover intentions, including poor quality relationships and the lack of support given to staff (Van Knippenberg et al, 2007; Lashinger \& Fida, 2014) as well as low wellbeing for US and Australian nurses (Brunetto et al., 2013). In each case, high wellbeing was associated with low turnover intentions. The following hypotheses replicate this finding for Bangladeshi nurses.

H3. Employee wellbeing is negatively related to turnover intention

\section{Mediation and Moderations}


The way that work harassment and wellbeing impact the relationship between LMX and turnover for Bangladeshi public and private sectors is not clear. There are two types of possible effects. First, work harassment moderates the association between LMX and wellbeing, and between LMX and turnover intentions. If this is the case then it means that work harassment affects the direction or strength of the relationship between LMX and wellbeing, and LMX and turnover intentions (Baron \& Kenny, 1986). Second, wellbeing mediates the association between LMX and turnover intentions, which means that employee wellbeing accounts for some of the negative relationship between LMX and turnover intentions. The two types of effects will be discussed further in the following paragraphs.

In terms of COR theory, wellbeing is the measure of an individual's personal resources and is affected by both personal (such as psychological capital) and organisational (such as LMX) factors. In particular, high LMX is associated with high wellbeing for Australian public sector nurses (Brunetto et al., 2016). In addition, high wellbeing is associated with low turnover intentions for Australian and US nurses (Brunetto et al., 2013). This means that if employees have high wellbeing (perhaps because of high personal resources), it may mediate the relationship between LMX and turnover intentions. According to COR theory, if wellbeing is high enough, we expect that the personal resources gained from high wellbeing will mediate the impact of LMX on turnover. The following hypothesis expresses this premise.

\section{H4. Employee wellbeing mediates the relationship between LMX and turnover intention} Moderation refers to a situation where the relationship between two variables depends on a third variable. As stated, previous research shows that high LMX is associated with high wellbeing and high work harassment is associated with low wellbeing. In the context of Bangladeshi nurses, we propose that high work harassment results in employees feeling overworked, under-resourced, and that overall their employing organisation's expectations are unrealistic. In such a work environment, even if employees have a good relationship with their 
manager, it is expected that high work harassment will reduce the positive impact of LMX and employee wellbeing, and decrease the negative impact of LMX on turnover intention. Moreover, we expect that work harassment will moderate the impact of LMX because a manager is considered an agent of the organisation (Eisenhardt, 1989), and thus unrealistic expectations may flow through the manager to the nurses. Therefore, if a nurse thinks expectations are unrealistic, they may associate such expectations with the manager, which when work harassment is high will reduce the positive influence of the manager on employee wellbeing and reduce the negative affect on turnover intentions.

\section{H5. Work harassment moderates the relationship between LMX and wellbeing}

\section{H6. Work harassment moderates the relations between LMX and turnover intentions}

\section{The public-private context}

The context of this research is a comparison of the public and private sector work environment for Bangladeshi nurses. The public sector in Bangladesh is highly bureaucratic and hierarchical, and is described as being over-governed (micro-managed and autocratic) with insufficient or inappropriate governance (subject to corruption) (Ahmed \& Shirahada, 2017). This means that there is a lack of separation between the official practices of public sector officials and personal interests, and a growing realisation that recent reforms, whilst in theory about re-distributing resources from the rich to the poor, did not achieve the stated goals (Khan et al, 2014). Also despite specific legislation promoting equal rights for women, reforms have not made much of an indentation on the glass ceiling imposed upon women by the dominant culture in play (Jamil, Aminuzzaman \& Hoque, 2013). According to Ahmad et al. (2007) (cited in Ghuman \& Singh, 2013) reforms were supposed to provide greater access to healthcare for the poor. However, in practice, the rich were given greater access to resources, and nurses were dissuaded against going to poorer regions because of inadequate infrastructure facilities. In 
summary, reforms did not achieve a greater focus on efficiency or achieving outcomes. Hence, LMX, work harassment, wellbeing and turnover is expected to be worse for public sector nurses. The hypothesis is:

H7: Private sector employees perceive higher LMX and wellbeing and lower work harassment and turnover compared with public sector nurses.

\section{Method}

\section{Sample and procedure}

In the first instance, nurses who had completed an undergraduate and/or postgraduate nursing qualification from the Grameen Caledonian College of Nursing, Dacca, Bangladesh were invited to complete the survey. In total, 191 useable surveys were returned (redemption rate of $62 \%$ ). Additionally, 126 useable surveys were received from nurses working in two public sector hospitals in Dacca, Bangladesh.

In total, 317 useable surveys were obtained, 131 from the public sector and 186 from the private sector. The demographic data depicts that unlike the ageing nursing workforce in many OECD countries (World Health Organization, 2018); the majority of nurses sampled in the Bangladesh public and private sectors were less than 31 years of age. As well, many have less than one-year employment with their current hospital, with the majority having less than 5 years employment in their current hospital. In the public sector, there were 34.4 per cent males $(n=45)$ and 65.6 per cent females $(n=86)$, which is a stark comparison to the private sector with 4.3 per cent males $(n=8)$ and 95.6 per cent females $(n=178)$.

\section{Measures}

All items for each scale were scored on a six-point Likert scale from 1 (strongly disagree) to 6 (strongly agree). The reliability for each scale is depicted in Table 1. 
Leader-member exchange. To measure a nurse's relationship with their manager, we used the Leader-Member Exchange (LMX) instrument developed by Graen and Uhl-Bien (1995). An example of an item is 'My manager is satisfied with my work.'

Employee wellbeing. We measured an employee's sense of their wellbeing with the four items from Brunetto, Farr-Wharton, and Shacklock (2011). A sample item includes 'Overall, I am reasonably happy with my work life.'

Turnover intentions. To identify nurse intentions to leave their employing organisation, we used three-items adopted from Meyer, Allen, and Smith (1993) scale of turnover intentions, including, 'I frequently think about leaving my current employer.'

Work harassment. Rayner and Cooper (2006) developed a scale to measure task-attack, personal-attack and intimidation. To measure work harassment, we used three items that examine 'task-directed attack,' including the item '[This hospital] sets unrealistic targets.'

Control variables. Many variables within the nursing and turnover intention literature may covary with turnover intentions. We controlled for tenure within a hospital because research has found those with longer tenure have a lower intention to leave their employing organisation (Ng \& Feldman, 2009b). Although there is limited agreement in the literature, we also controlled for gender, with one study suggesting women quit their job less than their male counterparts (e.g., Lyness \& Judiesch, 2001), and a larger scale study of 475,000 professionals and managers concluding women were more likely to voluntarily leave. Finally, we controlled for age because of $\mathrm{Ng}$ and Feldman's (2009a) postulation that older employees typically develop stronger ties to their colleagues and their employing organisations.

Data analysis

To test a moderated mediation model, we used SPSS v.25 and the PROCESS Macro by Andrew Hayes (Hayes, 2018). The PROCESS macro is a better alternative to test mediation 
when compared to approaches such as that prescribed by Baron and Kenny (1986), as there is no requirement for the data to be normally distributed. Within the PROCESS macro, we used model 8 and applied 95 per cent bootstrap confidence intervals across a sample of 5000. To confirm the factor structure and to account for common method variance, we undertook a confirmatory factor analysis of the public and private sectors. To check and control for common method variance, we included a common latent factor (Podsakoff, Mackenzie, Lee, \& Podsakoff, 2003).

\section{Results}

\section{Descriptive statistics and intercorrelations}

Table 1 depicts the descriptive statistics, scale reliability and intercorrelations. Notably, LMX did not significantly influence turnover intentions in the public sector. Although not significant, the $p$-value was nearing statistical significance $(p=0.06)$.

\section{Confirmatory factor analysis}

The hypothesised measurement model resulted in a poor fitting model for the private $(\mathrm{CMIN} / \mathrm{DF}=2.330, \mathrm{RMSEA}=0.085, \mathrm{CFI}=0.886$ and $\mathrm{SRMR}=0.066)$ and public $(\mathrm{CMIN} / \mathrm{DF}$ $=3.222, \mathrm{RMSEA}=0.131, \mathrm{CFI}=0.857$ and $\mathrm{SRMR}=0.081)$ sectors. We removed three $\mathrm{LMX}$ questions (numbers $4,6, \& 7$ ) and wellbeing question 4 , based on factor loadings below 0.5 and high modification indices. The modified measurement model fit the data well for the private $(\mathrm{CMIN} / \mathrm{DF}=3.222, \mathrm{RMSEA}=0.131, \mathrm{CFI}=0.857$ and $\mathrm{SRMR}=0.081)$ and public $(\mathrm{CMIN} / \mathrm{DF}=3.222, \mathrm{RMSEA}=0.131, \mathrm{CFI}=0.857$ and SRMR $=0.081)$ sectors. We tested a number of alternate models to establish the distinctiveness of the variables and the modified measurement model. As depicted in Table 2, the fit of the alternate models was significantly worse when compared with the modified measurement model for the private and public sectors.

The addition of a common latent factor (CLF) to the modified measurement model (model 4) for the private and public sectors resulted in improved model fit. In addition, a chi- 
square difference test for each sample revealed that the CLF model was statically different from the modified measurement model, and thus common method variance may explain a significant portion of the variance. To identify where common method variance was problematic, we compared the standardised factor loadings of the modified measurement model and the CLF model. To the best knowledge of the authors, there are no published cut-off values for the comparison of standardised factor loadings. We considered any loading difference above 0.20 to be excessively large and retained the CLF control. The final measurement model for the private and public sector include the bias-corrected estimates (i.e., controlling for common method bias). Specifically, to be able to test the hypotheses within the PROCESS macro, we imputed the bias-correct composite variables from AMOS into SPSS.

\section{Testing the hypotheses}

Figure 1 depicts the results for hypotheses 1-3 for the public and private sector. LMX predicts turnover $(\mathrm{H} 1)$ for private sector employees only and LMX predicts wellbeing $(\mathrm{H} 2)$ for both public and private sector and wellbeing predicts turnover intentions (H3) for public sector employees only. There was support for the acceptance of hypothesis 4 (mediation), with a significant indirect effect SE=-.21, CI (-.42,-.10). As mentioned, the direct effect between LMX and turnover intentions was not significant, providing support that wellbeing completely mediates the relationship between LMX and nurse turnover intentions in the Bangladesh public sector. We also found work harassment did not moderate the relationship between LMX and turnover intentions (i.e., rejecting hypothesis 6), but, as depicted in Figure 2, work harassment did moderate the path between LMX and employee wellbeing (i.e., providing support for the acceptance of hypothesis 5). Specifically, high work harassment reduced the influence of LMX on employee wellbeing.

In relation to the private sector, wellbeing did not directly relate to turnover intentions, so we rejected hypothesis 2 . Due to the rejection of hypothesis 3 , and insignificant indirect 
effects, we rejected hypothesis 4 . Finally, work harassment did not moderate the relationship between LMX and wellbeing, or LMX and turnover intentions. As such, we rejected hypotheses 5 and 6 .

\section{Discussion}

This paper examines the impact of management on work harassment, wellbeing and turnover of public and private nurses in Bangladesh. One factor which has been identified as having a negative impact, especially in the public sector, is work harassment and in developed countries, the manager either alleviates the situation or makes it worse (Brunetto et al, 2016b). However, research in Brazil found that management did not have the same impact compared with Anglo-American countries such as UK and Australia (Brunetto et al, 2015), hence, we examined the impact of management on public and private nurses' perceptions of work harassment in Bangladesh. Additionally, since, private sector nurses operate in hospitals that have attempted to implement some typically western HR systems, it seems likely that turnover should be less because the influence of culture (corruption etc) should be less.

In terms of the first and second hypotheses, previous research had already shown that effective management is associated with high wellbeing and low turnover intentions for public and private sector employees in Anglo-American countries (Martin et al., 2016; Brunetto et al., 2018, 2013). The findings from this research support previous research showing that high LMX is associated with high wellbeing for both public and private sector nurses in Bangladesh. The role of the manager is to act as a source of support when workloads are physically and mentally draining - hence in the ideal situation, they provide a barrier to stress, in turn supporting high wellbeing (Lashinger \& Fida, 2014). However, high LMX is only associated with low turnover for private sector nurses in Bangladesh. One explanation is that public sector nurses are unlikely to have a modern HR system in place (of which a basic manager-employee relationship is an important cornerstone), and instead, the traditional culture may be more 
important in determining whether employees stay or leave (Ahmed \& Shirahada, 2017; Khan et al., 2014). Khan et al (2014) argues that in Bangladesh, the policy may be similar to a developed country, but the implementation of HR policies has been challenging, especially in the public sector. Hence, it seems likely that family ties and corruption still dominate decision making, in turn negating the role of managers.

Additions, the findings suggest that in contrast to the role of managers of professionals in Anglo-American countries, who play two distinct (sometimes-contradictory) roles professional hybridity, firstly developing nurses' professional skills and knowledge, while still acting as their line manager (Noordegraaf, 2015); the role of public sector managers in Bangladesh is unclear because nurses' wellbeing is determined by how work harassment mediates the relationship between LMX and wellbeing. One possible reason is that their managers are not engaging in professional activities such as socialising them into the professional norms and values associated with nursing. Khan et al (2014) suggests that in countries like Bangladesh, increased professionalization of the Bangladeshi public sector has failed to change 'who gets what' and how workplaces are organised (Jamil, 2013). Hence LMX may not be based on professional factors and the managers may not act as positive antecedents of nurses' wellbeing as is the case in developed countries (Martin et al, 2016). However, further research should examine why a significant relationship was absent.

Regarding hypothesis 3, previous research had already shown that high wellbeing was associated with low turnover intentions for nurses in the USA and Australia (Brunetto et al., 2016). In terms of COR theory, high wellbeing is an important personal resource that employees want to develop, and conserve when required (Hobfoll 2011). The findings from this research are consistent with past research for public sector Bangladeshi nurses only. More research is required to investigate the relationship for private sector nurses. It may be that the traditional culture has a role to play in better understanding the relationship between wellbeing 
and turnover for Bangladeshi nurses working in the private sector (Khan et al., 2014). Such insight may better explain how employees build wellbeing in the workplace, and importantly, why they stay even in workplace enrivonments that deplete their wellbeing.

The mediation and moderation processes shed further light on understanding the differences in the public and private workplaces for nurses in Bangladesh. Past research shows that work harassment is a product of the era of austerity, which now governs the work lives of many professionals in the caring industry, especially in the public sector in developed countries. However, very little is known about how reforms (if any?) have affected the professional workplace in developing countries. In ideal circumstances, professionals expect their line managers to mediate unrealistic organisational work demands and provide appropriate and sometimes-extra support resources to ensure both task completion and professional development occurs (Farr-Wharton et al., 2011). In this study, work harassment does mediate the relationship between LMX and wellbeing for public sector nurses, but not for private sector nurses. Over half of public sector Bangladeshi nurses' wellbeing is explained by how work harassment mediates the relationship between LMX and wellbeing.

While previous research shows that LMX predicts work harassment (Farr-Wharton et al., 2018), and work harassment predicts employees' wellbeing (Xerri et al., 2016), this paper adds new evidence about the role of work harassment in moderating the relationship between management and wellbeing of public sector nurses in Bangladesh. More specifically, in the public sector, when work harassment was low, management had a greater positive influence on employee wellbeing. These results provide new evidence about the influence of work harassment on the well-established association between management practices and wellbeing. This means that the role of the manager is just as important in developing countries such as Bangladesh as it is in developed countries, although culture may affect how it works. It also aligns with COR theory in that those with fewer resources are less able to apply resources to 
develop other resources, which suggests that those managers who support their nurses impact their decision to stay by improving their wellbeing. However, this is the case for public sector nurses only.

In contrast, LMX explains just under a quarter of private sector nurse wellbeing, and work harassment has no significant impact on this association. Further, there is no evidence of work harassment moderating the relationship between LMX and turnover in this study, although, high LMX, work harassment and wellbeing does explain just under a third of the turnover intentions of public sector Bangladeshi nurses. In the case of private sector Bangladeshi nurses, work harassment alone explains well over a third of their turnover intentions. This suggests that the manager may not have the power to affect how work harassment influences turnover intentions for private sector nurses. This is in contrast to research findings in the Anglo-American countries and may suggest a significantly different work context for this private sector nurses working in one of the least developed countries in the world. The findings provide a platform for more research about management issues facing public and private professionals such as nurses in developing countries such as Bangladesh.

The last hypothesis examined the differences between the public and private sector nurses' level of satisfaction with LMX, work harassment, wellbeing and turnover. The belief was that private sector nurses would perceive higher LMX and wellbeing and lower work harassment and turnover intentions compared to their private sector counterparts. The hypothesis was based on information that public sector reforms have not been successfully implemented, the public sector in Bangladesh is poorly resourced, and nurses are actively dissuaded from going to poorer regions because of inadequate infrastructure facilities (Jamil, 2013). However, the hypothesis was rejected because, across every factor, public sector nurses were more satisfied with management, had higher wellbeing, perceived lower work harassment and turnover intentions. One explanation using COR theory is that private sector nurses' 
wellbeing is being conserved and developed from other resources not examined in this paper. Future studies should further examine the antecedents, mediators and moderators relating to work harassment.

In reviewing this study, there are some limitations to consider. First, the cross-sectional data was susceptible to common method bias (Podsakoff et al., 2003). However, as mentioned, a common latent factor was included, and several items that were explained by common method variance were controlled. Nonetheless, future research should examine work harassment over time to improve insight and design relating to common method bias. Second, although a study on Bangladeshi nurses provides some insight into work harassment in developing countries, further research is required about other developing countries. Finally, the findings are limited by the sample size for each sector. Further research should be undertaken to increase the sample size, which will improve the stability of the results, and provide enough statistical power to examine more complex research models, including a thourough examination of work harassment antecedents, mediators and moderators.

\section{Conclusion}

The findings suggest that work harassment is prevalent in the Bangladesh public and private nursing profession. This study used a COR theoretical framework to compare the impact of LMX and work harassment on the wellbeing and turnover intentions of Bangladeshi public and private nurses. Using COR theory, we found that LMX is a positive resource caravan passageway that can build wellbeing for public sector employees; however, the association was not significant for private sector nurses. Hence, COR theory principle 2 is relevant for public sector employees because the manager provided enough of a cushion to form a barrier against the negative influence of work harassment on wellbeing (Hobfoll, 2001). The consequence of the poor environmental conditions, such as work harassment, is that it does affect how public 
sector nurses in Bangladesh are able to take advantage of the relationship with their manager. However, there was no evidence that employees who perceive high support from their managers are less likely to be negatively influenced by work harassment (Hobfoll, 2011). The theory was useful for examining how work harassment affects public and private sector nurses differently.

The implication of this research relates directly to public sector management. For example, the impact of management is different in Bangladesh compared with past AngloAmerican studies, and one factor is likely to be the traditional cultural stronghold, especially the impact of ties and corruption, which affects nurses' turnover intention, especially in public sector nurses areas (Ahmed \& Shirahada, 2017). Additionally, there are significant differences in how public and private sector nurses respond to, and cope with work harassment. Hence, simply transplanting management models from developed countries for managing healthcare in developing countries may not yield its intended benefits. More research is required to better understand how managers affect how professionals work in developing countries. 


\section{References}

Ahmed, T \& Shirahada, K (2017) Toward a sustainable healthcare service system in a limited resource context: Case study of Bangladesh rural advancement committee's healthcare system, International Journal of Healthcare Management,

DOI: $10.1080 / 20479700.2017 .1389512$

Baron, R. M., \& Kenny, D. A. (1986). The moderator-mediator variable distinction in social psychological research: Conceptual, strategic, and statistical considerations. Journal of Personality and Social Psychology, 51(6), 1173-1182.

Branch, S., Ramsay, S., \& Barker, M. (2013). Workplace bullying, mobbing and general harassment: A review. International Journal of Management Reviews, 15, 280-299

Brunetto, Y., Farr-Wharton, R., \& Shacklock, K. (2011). Using the Harvard HRM model to conceptualise the impact of changes to supervision upon HRM outcomes for different types of public sector employees International Journal of Human Resource Management 22(3), 553-573.

Brunetto, Y., Rodwell, J., Shacklock, K., Farr-Wharton, R., \& Demir, D. (2016a). The impact of individual and organizational resources on nurse outcomes and intent to quit. Journal of advanced nursing, 72(12), 3093-3103.

Brunetto, Y., Xerri, M., Shriberg, A., Farr-Wharton, R., Shacklock, K., Newman, S., \& Dienger, J. (2013). The impact of workplace relationships upon engagement, wellbeing, commitment and turnover for nurses in Australia and USA. Journal of advanced nursing, 69(12), 27862799.

Brunetto, Y., Xerri, M., Trinchero, E., Beattie, R., Shacklock, K., Farr-Wharton, R., \& Borgonovi, E. (2018). Comparing the impact of management on public and private sector nurses in the UK, Italy, and Australia. Public Management Review, 20(4), 525-544.

Brunetto, Y., Xerri, M., Trinchero, E., Farr-Wharton, R., Shacklock, K., \& Borgonovi, E. (2016b). Public-Private Sector Comparisons Of Nurses' Work Harassment Using Set: Italy and Australia. Public Management Review, 18(10), 1479-1503. doi:DOI:10.1080/14719037.2015.1114136

Brunetto, Y., Shacklock, K., Teo, S., Farr-Wharton, R., \& Nelson, S. (2015). Nurses' managers, learning options and organisational commitment: Australia, Brazil and England. Journal of Nursing Management. $23(8), 1029-1038$

Dick, G. P. (2010). Can the organisation and supervision environment influence both bullying and organisational commitment? Evidence from a police force survey. Kent University Working Paper Series 196.

Diefenbach, T. (2009). New public management in public sector organizations: the dark sides of managerialistic 'enlightenment'. Public Administration, 87(4), 892-909.

Einarsen, S. (2000). Harassment and bullying at work: A review of the scandinavian approach. Aggression and Violent Behavior, 5(4): 379-401

Einarsen, S., Hoel, H., \& D. Zapf, D. eds. (2011). Bullying and Harassment in the Workplace: Developments in Theory, Research and Practice ( $2^{\text {nd }}$ Edition). CRC Press, Taylor \& Francis Group, Boca Raton, Florida.

Einarsen, S., \& Nielsen, M. (2015). Workplace bullying as an antecedent of mental health problems: A five-year prospective and representative study. International Archives of Occupational and Environmental Health. 88: 131-142

Eisenhardt, K. M. (1989). Agency theory: An assessment and review. Academy of Management Review, 14(1), 57-74.

Farr-Wharton, R., Brunetto, Y., \& Shacklock, K. (2011). Professionals' manager-subordinate relationships, autonomy and commitment in Australia: a leader-member exchange theory perspective. The International Journal of Human Resource Management, 22(17), 3496-3512. 
Forgeard, M. J., Jayawickreme, E., Kern, M. L., \& Seligman, M. E. (2011). Doing the right thing: Measuring wellbeing for public policy. International journal of wellbeing, 1(1), 79-106.

Ghuman, B. S., \& Singh, R. (2013). Decentralization and delivery of public services in Asia. Policy and Society, 32, 7-21

Graen, G., \& Uhl-Bien, M. (1995). Relationship-based approach to leadership: Development of leader-member exchange (LMX) theory of theory over 25 years: Applying a multi-level multidomain perspective. Leadership Quarterly, 6(2), 219-247.

Halbesleben, J. R., Neveu, J.-P., Paustian-Underdahl, S. C., \& Westman, M. (2014). Getting to the "COR" understanding the role of resources in conservation of resources theory. Journal of management, 40(5), 1334-1364.

Hayes, A. F. (2018). Introduction to mediation, moderation, and conditional process analysis: A regression-based approach. New York: Guilford Press.

Hobfoll, S. E. (1989). Conservation of resources: A new attempt at conceptualizing stress. American psychologist, 44(3), 513-524.

Hobfoll, S. E. (2001). The influence of culture, community, and the nested-self in the stress process: advancing conservation of resources theory. Applied psychology, 50(3), 337-421.

Hobfoll, S. E. (2011). Conservation of resource caravans and engaged settings. Journal of occupational and organizational psychology, 84(1), 116-122.

Jamil, I., Aminuzzaman, S., \& Haque, S. (2013) Introduction: Governance in South, Southeast, and East Asia Public Organization Review 13 (4) 341- 347

Khan., R, Miah., M, \& Manzoor., A (2014). Human Resource Management Practices: A Case Study of South Asian Countries IBT Journal of Business Studies 9(1), 83-101

Lyness, K. S., \& Judiesch, M. K. (2001). Are female managers quitters? The relationships of gender, promotions, and family leaves of absence to voluntary turnover. Journal of applied Psychology, 86(6), 1167-1178.

Mahdy, H. A. (2009) Reforming the Bangladesh healthcare system, International Journal of Health Care Quality Assurance, 22(4), 411-416.

Martin, R., Thomas, G., Guillaume, Y., Lee, A. \& Epitropaki, O. (2016). Leader-member Exchange

(LMX) and Performance: A Meta-analytic Review. Personnel Psychology, 69, 67-121.

Meyer, J. P., Allen, N., \& Smith, C. A. (1993). Commitment to organizations and occupations: extension and test of a three-component conceptualization. Journal of applied Psychology, 78(4), 538-551.

$\mathrm{Ng}, \mathrm{T}$. W., \& Feldman, D. C. (2009a). Age, work experience, and the psychological contract. Journal of Organizational Behavior, 30(8), 1053-1075.

$\mathrm{Ng}, \mathrm{T}$. W., \& Feldman, D. C. (2009b). Re-examining the relationship between age and voluntary turnover. Journal of Vocational Behavior, 74(3), 283-294.

Noordegraaf, M. (2015) Hybrid Professionalism and Beyond: (New) Forms of Public Professionalism in Changing Organizational and Societal Contexts, Journal of Professions and Organization, 2, 187-206.

Podsakoff, P. M., MacKenzie, S. B., Lee, J.-Y., \& Podsakoff, N. P. (2003). Common method biases in behavioral research: a critical review of the literature and recommended remedies. Journal of applied Psychology, 88(5), 879-903.

Rayner, C., \& Cooper, C. L. (2006). Workplace bullying. In K. E. Kelloway, J. Barling, \& J. J. H. Jr. (Eds.), Handbook of workplace violence. Thousand Oaks: Sage Publications.

Laschinger, H.K.S., Fida, R., (2014). New nurses' burnout and workplace wellbeing: the influence of authentic leadership and psychological capital. Burnout Research 1 (1), 19-28

Seligman, M.E., (2012). Flourish: A visionary new understanding of happiness and well-being. New York: Simon and Schuster.

Tummers, L., Vermeeren, B., Steijn, B., \& Bekkers, V. (2012). Public professionals and policy implementation: Conceptualizing and measuring three types of role conflicts. Public Management Review, 14(8), 1041-1059. 
Tummers, L., Brunetto, Y., Teo, S., (2016) Workplace aggression: Introduction to the special issue and future research directions for scholars, International Journal of Public Sector Management, 29 (1), 2 - 10

Van Knippenberg, D., Van Dick, R., \& and Tavares, S. (2007). Social Identity and Social Exchange: Identification, Support, and Withdrawal from the Job. Journal of Applied Social Psychology, 37(3), 457-477.

Van der Doef, M. and Maes, S., 1999. The job demand-control (-support) model and psychological well-being: a review of 20 years of empirical research. Work \& stress, 13(2), pp.87-114.

World Bank. (2016). Overview of Bangladesh. Retrieved from http://www.worldbank.org/en/country/bangladesh/overview

World Health Organization. (2016). World Health Statistics 2016: Monitoring Health for the Sustainable Development Goals (SDGs). World Health Organization. Retrieved from http://www.who.int/gho/publications/world health statistics/2016/en/

World Health Organization. (2018). Nursing and Midwifery Fact Sheet February 2018. Retrieved from http://www.who.int/mediacentre/factsheets/nursing-midwifery/en/

Xerri, M., Farr-Wharton, R., Brunetto, Y., \& Lambries, D. (2016). Work harassment and local government employees: Australia and USA. International Journal of Public Sector Management, 29(1), 54-71.

Zapf, D., Escartin,J., Einarsen, S., Hoel, H. \& Vartia, M. (2011). Empirical findings on the prevalence and risk groups of bullying in the workplace. In Bullying in the Workplace; Developments in Theory, Research and Practice edited by Einarsen, S., Hoel, H., Zapf, D., Cooper. C. CRC press, Taylor \& Francis group, Boca Rata 
Table 1. Correlation matrix

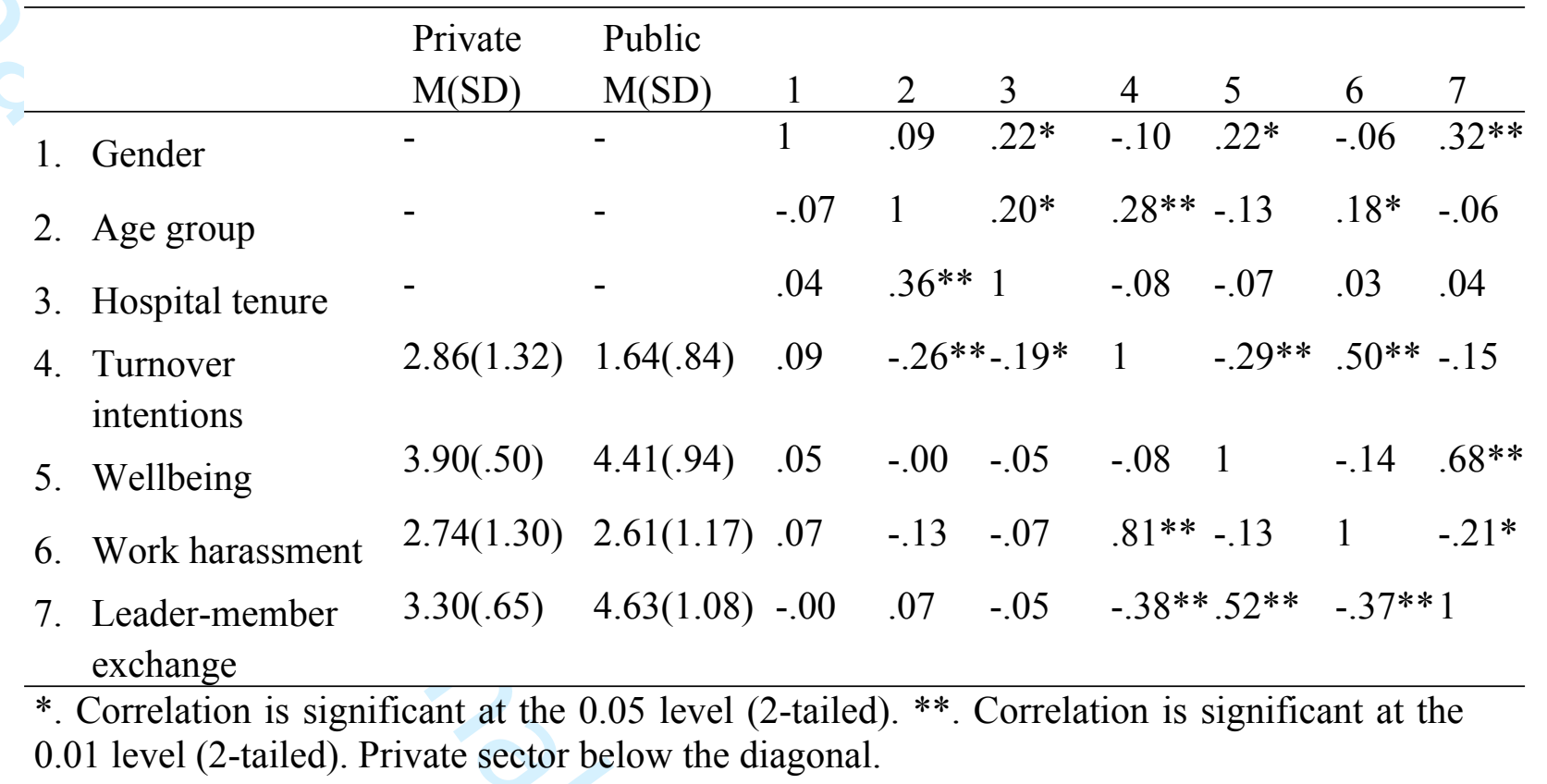

Table 2. Comparison of alternate measurement models

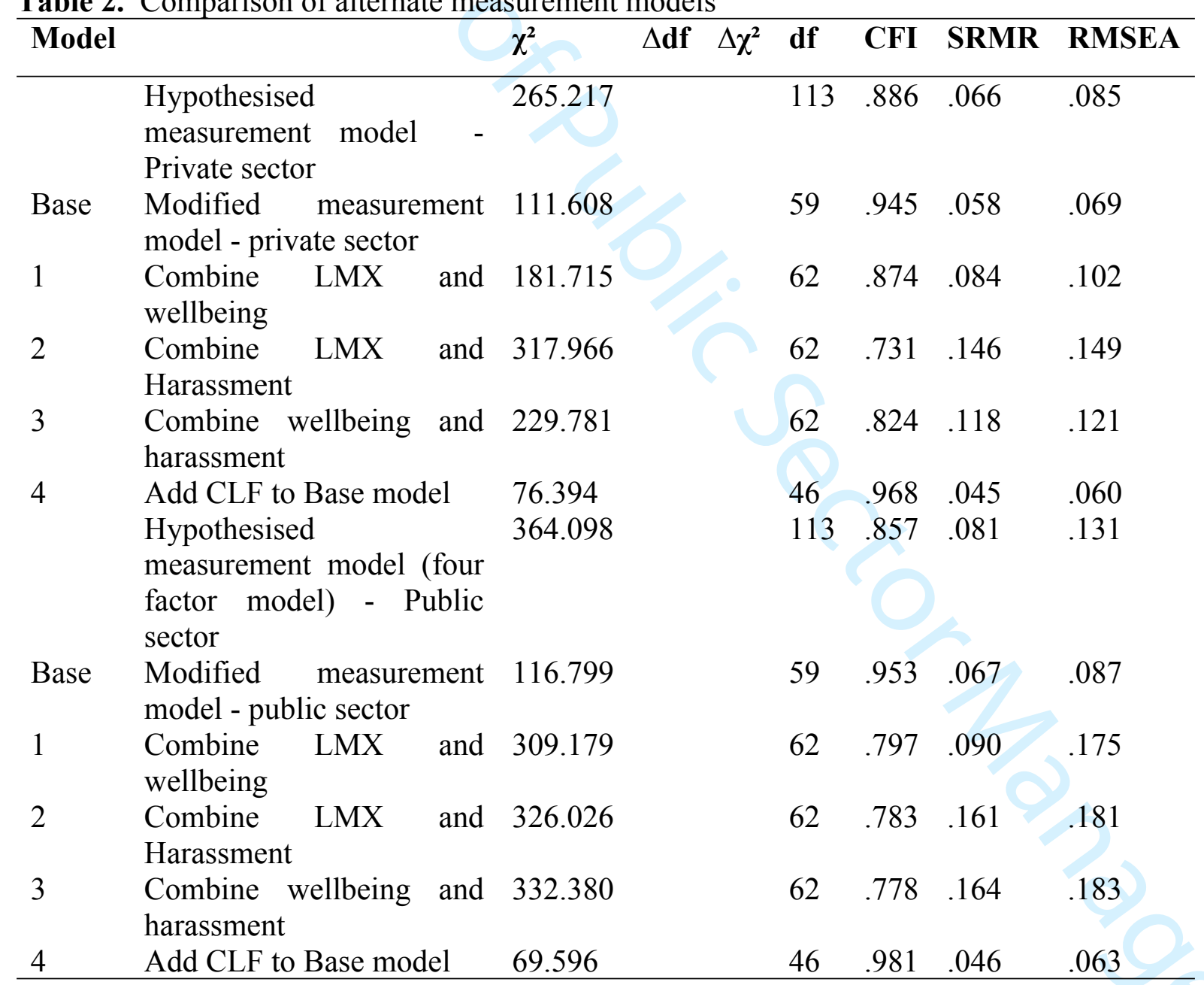




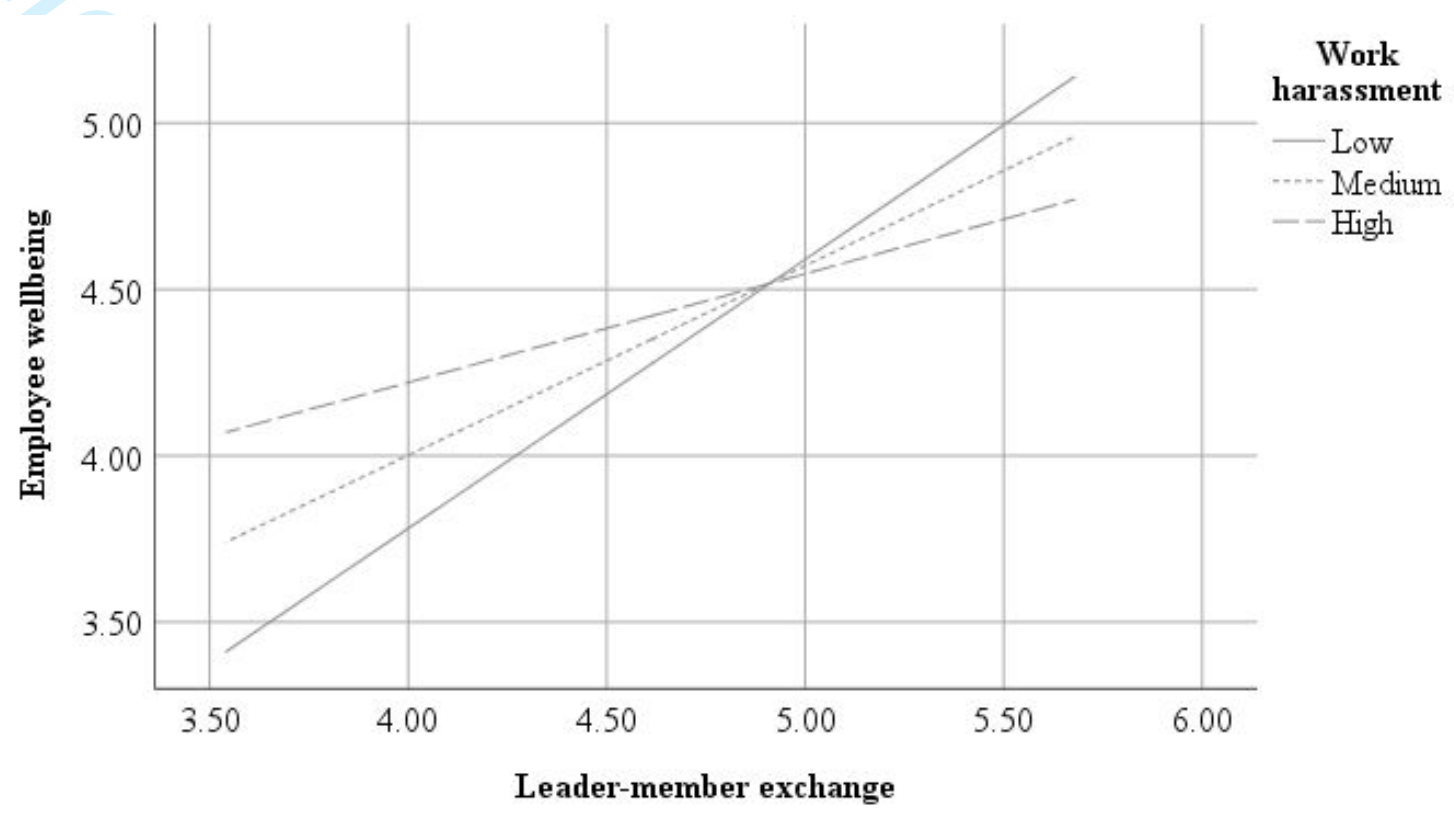

Figure 2. Moderation results - work harassment moderates the relationship between LMX and employee wellbeing. Note: Work harassment standardised for moderation analysis 


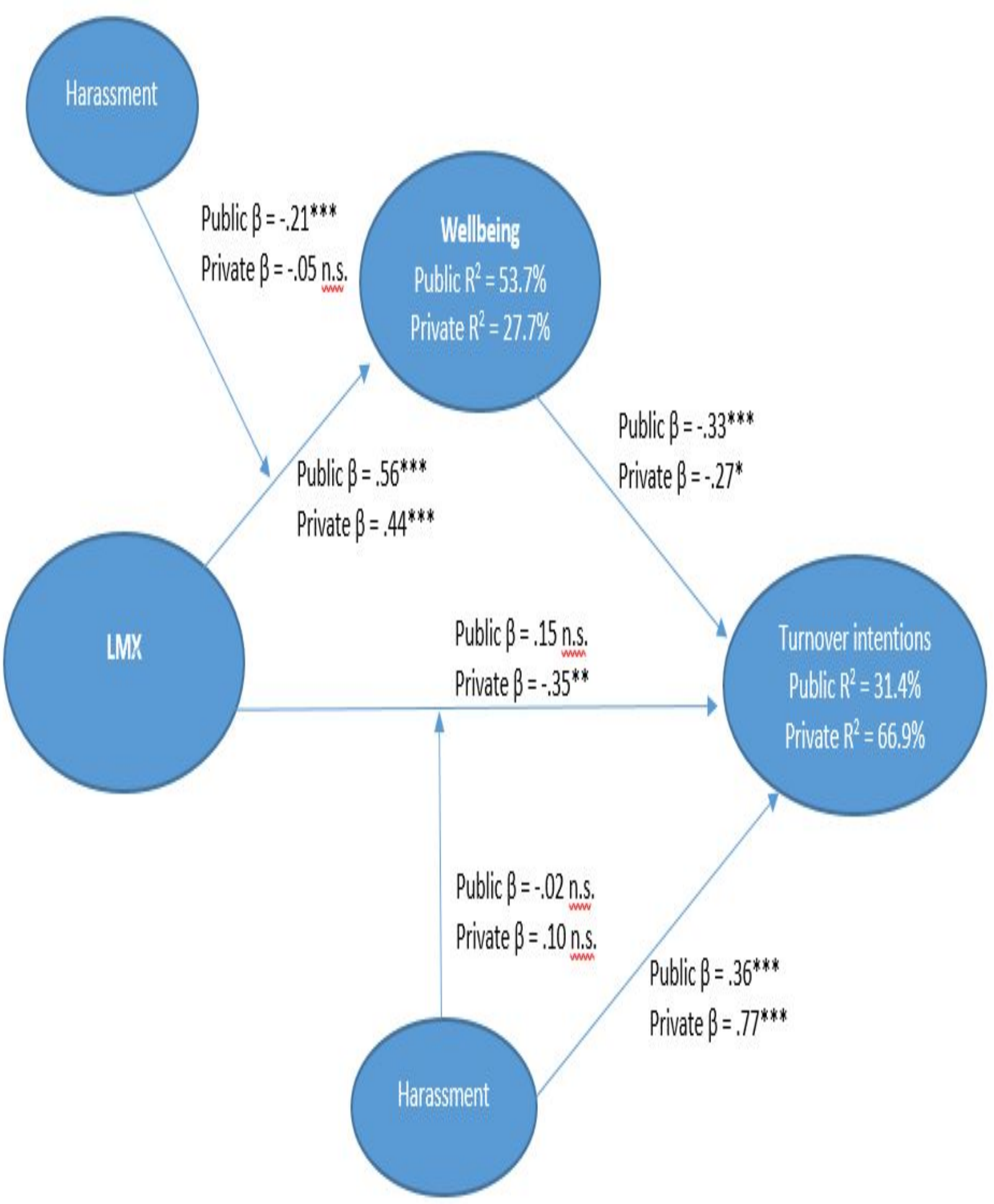




\title{
Comparing the impact of management on public and private nurses in Bangladesh
}

\begin{abstract}
Purpose: This paper uses theory as a lens for comparing the impact of line management on Bangladeshi public and private nurses' perception of work harassment, wellbeing and turnover intentions where Anglo-American and European management models have been superimposed on an existing different culture.
\end{abstract}

Design: Survey data was collected from 317 Bangladeshi nurses' (131 from the public sector and 186 from the private sector). Structural Equation Modelling was used for analysis.

Findings: Good management was associated with high wellbeing, and $\underline{H}$ high work harassment was associated with low wellbeing and. However, management practices explained approximately a quarter of private sector nurse's wellbeing., and work harassment had no impact on this association. In total, management, work harassment and wellbeing explained approximately a third of the turnover intentions of public sector nurses, whereas only work harassment explained approximately a third of private sector nurses' turnover intentions.

Limitation; Cross-sectional data is susceptible to common method bias. A common latent factor was included, and several items that were explained by common method variance were controlled. Further, the findings are limited by the sample size from one sector and the use of only one developing country.

Implications: It is a waste of resources to transplant Anglo-American and European management models to developing countries without understanding the eultural context impact on nurses' outcomes.

Originality: Anglo-American and European management models and theories are not easily transferable to the public sector Bangladesh context probably because of the impact of ties and 
corruption. Line mManagement is a positive resource that can build wellbeing for public sector employees only.

Keywords: public-private sector, line management, well-being, work harassment, turnover intentions 


\section{Comparing the impact of line management on public and private nurses in Bangladesh}

Public sector management models do not easily translate to best practice in developing countries, mainly because the conditions are too different. For example, in contrast to developed countries, there are more doctors than nurses in Bangladesh and the demand for nurses' services exceeds supply whilst continuing to grow in line with population growth: hence they work in high-pressure environments (World Health Organization, 2018; World Bank, 2016). As a consequence, this paper examines whether Bangladeshi nurses perceive work harassment similarly to other public and private nurses in developed countries (Brunetto, Xerri, Trinchero, Farr-Wharton, Shacklock, Borgonovi, 2016) and whether this affects their turnover intention. Dick (2010) refers to work harassment as a form of workplace bullying but suggests that it differs from bullying. Specifically, an employee is exposed to work harassment when their employing organisation unreasonably monitors their actions, sets excessively high workloads, and has stringent accountability standards (Brunetto et al, 2016b). As such, the organisation and its managers are the instigators of work harassment.

Work harassment has been identified as a growing problem for public sector managers in developed countries (Tummers, Brunetto \& Teo 2016). For example, Brunetto et al. (2016b) compared work harassment of nurses in Australia and Italy and found significant parallels in their perceptions. More recent research identified a growing presence and acceptance of work harassment in US and UK hospitals (Farr-Wharton, Brunetto \& Xerri, 2019). The delivery of health services has been significantly affected by the cost-constraint that mandated the depth and breadth of reforms over the past four decades which has resulted in the expectation that employees will 'do more with less' (Diefenbach, 2009). However, the situation is even more pronounced in Bangladesh, where the shortages in nurses, especially in the public sector and in particular, in rural areas, is a growing problem (Ahmed \& Shirahada, 2017). Specifically, there are high levels of resource inadequacy, with widespread shortages in basic nursing 
equipment and a lack of an effective nurse management systems (World Health Organization, 2016). Such inadequacies in the Bangladesh system are likely to create a potential haven for work harassment.

The shortage of public sector nurses in Bangladesh is increasingly dire, and in some cases, the families of patients have to act as carers (Mahdy, 2009). If work harassment is not managed effectively, then it may negatively influence the profession's ability to attract new nurses, and retain them in the job. Whilst there is evidence of work harassment across different public sector occupations, such as local government and healthcare workers across different countries - Australia, Italy, USA (Tummers et al 2016; Xerri et al, 2016), there is no evidence as to whether work harassment is an issue in developing countries. In this study, we have two core aims. The first aim is : firstly, to develop insight into the impact of line management on outcomes such as work harassment, wellbeing and turnover intentions for nurses working in Bangladeshi hospitals. The and-secondly aim,is to develop a deeper understanding of the similarities and differences across the public and private sectors.

In relation to work harassment, in developed countries the theory argues that the role of the line manager for professionals is very important because of the dual role of the professional mentor and line manager within the organisational hierarchy. However, line managers of professionals are also increasingly faced with the issue of 'professional hybridity' (the sometimes-contradictory expectations that managers will both develop nurses' professional skills and knowledge and ensure task completion) (Noordegraaf, 2015). Never-the-less, a good line manager is one who can mediate the excessive demands of the hierarchy to ensure appropriate resourcing and support is available in relation to task completion and professional development (Farr-Wharton, Brunetto, \& Shacklock, 2011). Although past evidence has established the association between line management practices and wellbeing, and between line management practices and turnover intentions (Brunetto et al., 2013), to the best 
knowledge of the authors, there is no evidence about how work harassment may change these established relationships. As such, we aim to add to the existing evidence by examining the role of work harassment in affecting the association between satisfaction with line management and employee wellbeing, and in turn, turnover intentions within a developing country such as Bangladesh. Such evidence adds to the literature about the impact of line management on employee wellbeing and turnover intentions of nurses within developing countries, in this case, Bangladesh.

Managing both public and private sector employees in developing countries can be challenging as international HR systems are imported into existing culture-driven decisionmaking systems. Most managers were traditionally autocratic in their practices in South Asia (Khan, Miah \& Manzoor, 2014). However, in the last three decades, the service sector has undergone significant change mainly because of global forces and government reforms that have elevated the importance of human resources slowly, especially in terms of the significance of ensuring adequate qualifications and competencies for key positions. This change has led to the introduction of modern HRM and strategic systems, however, the new systems have not replaced the previous stronghold of culture in organisational decision-making. This means that family ties and corruption still dominate decision making, favouring some and disadvantaging the majority, and whilst those with strong international connections are likely to have basic HR policies and structures evident in countries such as the USA and UK, the difference is in their ability to implement those policies into practice (Khan et al., 2014, p 99). Hence, while Bangladeshi organisations may have specific policies about work hours and duties, in practice, there is probably greater scope for work harassment in Bangladesh compared with AngloAmerican countries such as the UK, USA and Australia.

This paper compares the relationship between line management, work harassment, wellbeing and turnover intentions of public and private sector nurses using a Conservation of 
Resources (COR) theoretical framework (Hobfoll, 1989). COR theory is typical of theories originally developed and tested within an Anglo-American and/or European context (history, culture) to explains employee outcomes regarding their ability to maintain and grow their personal resources such as health and wellbeing. However, it may not have as much relevance in explaining behaviour in developing countries. COR theorists argue that when the organisational environment erodes their personal resources through work harassment, their wellbeing is negatively impacted and this motivates them to protect their well-being by regulating their work practices. COR theorists argue that if extra support (e.g., from managers) is not available, then employees may offset perceived resource losses, and potential shortages, by reducing effort at work or leaving the organisation (Hobfoll, 2011). The research questions (RQs) guiding the study are:

$R Q$ 1: What is the impact of line management on work harassment, wellbeing and turnover of public and private sector Bangladeshi nurses?

$R Q$ 2: What are similarities and differences in the relationship between line management, work harassment, wellbeing and turnover for nurses in the public and private sector?

The next part details a relevant review of the literature and identifies hypotheses that reflect the gap in the literature.

\section{Background}

\section{Conversation of Resources (COR) theory}

The COR theory is ideal for understanding employee outcomes, especially in the face of resource adversity (Hobfoll 1989). The way COR theory works is via two principles and four Corollaries (Hobfoll, 2011). Principle one: a loss of resources likely has a bigger effect than acquiring new resources. Principle two: to gain new or more resources, employees must firstly spend time or energy (resources) in those activities likely to lead to more resources. Further, if employees believe they are likely to experience a loss of resources (such as a loss 
of wellbeing) they will likely engage in activities (sick leave/ look for another job) to protect themselves against falling wellbeing. The four corollaries comprise four assumptions include: (1) Firstly, the higher employees' perception of resources are, the easier it is for them to gain resources, whereas employees with fewer resources are more susceptible to resource loss, and less able to gain resources. (2) Secondly, itt is likely that an initial loss of resources will lead to further losses; whereas (3) it is likely that a resource gain will likely lead to further resources gains $_{-} ;$Fourthly, and (4) employees are likely to act defensively as a strategy to prevent further loss of resources (Halbesleben, Neveu, Paustian-Underdahl, \& Westman, 2014).

Drawing from COR theory, each organisation makes decisions that produce either positive or negative environmental conditions, called 'resource caravan passageways' (Hobfoll (2011). Under ideal conditions, an employee can build resources such as increased wellbeing by investing other resources at their disposal; in this case, effective supervision is a social resource (caravan passageway) an employee can draw upon. Whereas, if the resource caravan passageway is high levels of work harassment, employees have low resources and are thus less able to orchestrate resource gain. In this case, we propose that work harassment may result in employees that are less able to develop wellbeing with the social resource derived from the relationship with their manager.

Using COR theory to explain the relationship between management, work harassment, wellbeing and turnover, we argue that effective management practices is a positive resource caravan passageway that can build wellbeing, whereas work harassment is a negative resource caravan passageway that will influence an employee's ability to develop resources such as wellbeing. The consequences of the poor environmental conditions are likely to lead to increased turnover (Hobfoll, 2001). Using COR corollary 4, the issue will be whether the support from the manager is enough of a 'cushion' to form a barrier against the negative influence of work harassment on wellbeing, and the flow on to an intention to turnover. As per 
corollary 4 , those with a lack of resources are likely to act defensively to conserve against further resource loss. That is, those who lack resources are unable to apply resources to fight against resource loss (Hobfoll, 2011), and thus to conserve further loss, they may choose to eject themselves from the situation by intending to leave their employing organisation. In contrast, those employee with adequate resources are likely to be able to invest them in resource develop, which is likely to be evident by high wellbeing and low turnover intentions.

\section{Variables under examination}

\section{Work Harassment (resource caravan passageway)}

The term 'work harassment' is challenged by different groups of researchers and the same work has multiple meanings depending on the country. Originally, the term emerged from 'harassment' meaning “... all repeated behaviors [sic] which ... provoke, frighten, intimidate, or bring discomfort to the victim" (Brodsky (1976) cited in Einarsen, 2000; p382). However, the definition has moved on in different ways in different countries. For example, the Scandinavian researchers adopted different terms compared with the Anglo-American researchers for similar behavioural aggression and their remains no consensus about definitions for the different negative work behaviours (Branch, Ramsay, \& Barker, 2013; Einarsen, \& Nielsen, 2015). In terms of public sector HR in Anglo-American and/or European countries, work harassment has emerged as a negative work behaviour, similar to bullying or mobbing because it is characterised by repeated ill-treatment with negative consequences for the individual (Zapf, Escartin, \& Einarsen, 2011).

Originally, work harassment was conceptualised as part of bullying because of its negative impact on the individual (Raynor, 2002). More recently, work harassment is differentiated from bullying for three main reasons. Firstly, bullying is directed at an individual, whereas work harassment is about task completion and affects large numbers of front-line employees facing a chronic problem of demand outstripping supply, forcing them either to 
choose 'who get what' (or who misses out) and/or 'to do more with less' on a continual basis (Dick, 2010; Xerri, Farr-Wharton, Brunetto, \& Lambries, 2016). Secondly, bullying can involve physical or mental abuse directed at an individual, whereas work harassment is not personal. Instead, it is the result of a resourcing decision made originally by governments, and then by senior management in allocating resources to each department (Tummers, et al, 2016).

Thirdly, the incidence of work harassment relies on the power of professional principles to ensure patients/clients/customers are serviced, irrespective of organisational resourcing constraints. This is because universities and the professional bodies regulate entry into the professions and work practices are underpinned by specific norms and values (such as professional standards of service and effectiveness) (Farr-Wharton et al., 2011). This in turn that often brings them into conflict with organisational norms (task completion/ reduced per capita costs) leading to 'professional hybridity' (Noordegraaf, 2015). One of the outcomes for healthcare professionals is increasing stress from role conflict as professionals work overtime (often against organisational directions) (Tummers, Vermeeren, Steijn, \& Bekkers, 2012). Hence, work harassment is a type of negative workplace behaviour that is emerging as a growing HR problem, especially for healthcare professionals working in the public sector where austerity dominates resourcing decisions. In particular, Tummers et al. (2016) identified the rise in stress (and stress-related illnesses) emerging amongst professionals because of increasing work harassment, role overload and role ambiguity.

For healthcare workers, high demand is a worldwide phenomenon. In those countries where there is also a shortage of nurses, the workplace conditions are even more likely to enhance, rather than detract from work harassment. For example, Laschinger and Fida (2014) found that in Canada, even new graduate nurses were reporting high-stress levels leading to falling wellbeing and the worst cases, a steady rise in the incidence of high levels of burnout and turnover. Laschinger et al. $(2014$, p19) argue that the key contributors are heavy workloads 
and inadequate staffing that in turn, create the negative environmental conditions ripe for increasing job dissatisfaction, and:

"Similar to other helping professions, the prevalence of burnout in nursing is particularly high, because of the high emotional and physical demands of this work"

However, it may be the impact of cultural factors intervening for Bangladeshi nurses that negates the impact of work harassment if for example, they perceive the importance of helping pateints are over-riding the impact of high workloads on their wellbeing. This is what is unknown - do Bangladeshi employees such as nurses behave similarly to employees in developed countries? In terms of COR theory, high work harassment acts as a negative work behaviour that erodes personal resources. If an employee has a shortage of resources - they will likely see a reduction in their wellbeing. As such, principle two is enacted and consequently, employees seek to control the negative flow of wellbeing by looking for another job (as one example of acting defensively) (Hobfoll 2011). Unless there is a counterbalance of positive resources, for example - positive relationships with their managers that can buffer and negate the impact of work harassment, then employees' personal resources will be negatively affected.

\section{Employee Resources (resource caravans)}

\section{Manager Support - Leader-member exchange (a social support resource)}

Manager-subordinate relationships can be conceptualised via the LeaderMember Exchange (LMX) framework. LMX is a Social exchange theory (SET) variable because the potential benefits from effective workplace relationships far outweigh the initial outlay of good will. LMX argues that managers form different quality relationships with employees: for some their workplace interactions are meaningful and over time leads to mutual reciprocity (insiders). On the other hand, whereas for others the interactions are negative and 
consequently a relationship forms that is built only on the work contract specifications (Martin, Thomas, Guillaume, Lee, \& Epitropaki, 2016). The benefits of an effective LMX relationship were identified in a meta-analysis by Martin et al. (2016) as high role performance and high citizenship behaviour as well as low counteractive workplace behaviour. This relationship becomes even more important when it involves professionals because there is an added responsibility on the part of the manager to ensure the employee is socialised into the values, norms and practices associated with the profession and adheres to professional standards (Brunetto et al., 2018). However, research about public sector nurses in Brazil found that that the theories and "truths" accepted as the behaviour of employees such as nurses in developed countries does not always translate to the behaviour of nurses in BRIC countries. For example, Brunetto, Shacklock, Teo, Farr-Wharton \& Nelson (2015) found that managers had minimal impact on Brazilian nurses' behaviour for numerous reasons, including that there were different contracts in place with some nurses reporting to managers in the capital of Brazil, not the hospital, and the impact of corruption on nurses' actions in the workplace.

However, using COR theory, when LMX is high, it suggests that employees can access support from managers or someone in management. Hence, it is not surprising that previous research in developed countries suggests that high LMX is associated with high wellbeing and low turnover for nurses in Anglo-American and European countries such as Australia, Italy and the UK (Brunetto et al., 2018) as well as the USA (Brunetto et al., 2013). The reason as to why LMX is associated with high employee performance is because as Lashinger and Fida (2014) found in their research of Canadian nurses, high LMX indicates that nurses are receiving high levels of support from managers, which in turn provides a barrier to stress and helps maintain high employee wellbeing. However, the relationship has not been examined for public and private sector Bangladeshi nurses, where cultural influences on HR and workplace relationships do not necessarily follow the Anglo-American and/or European model. To test 
whether similar relationships are in play with Bangladesh for nurses, the following hypotheses are used.

\section{H1. LMX is negatively related to turnover intention \\ H2. LMX is positively related to employee wellbeing}

Employee well-being (an individual/personal resource)

'Well-being' is a general term with multiple definitions across different disciplines and there is no agreement on a definition except; however, that it is a multi-faceted concept (incorporating physical, job-related, psychological, social wellbeing), and each time it is used, it requires explicit defining in order to avoid confusion (Seligman, 2012). Employee well-being is defined as a composite state that encompasses a hedonic component (life satisfaction) and a eudaimonic component (living life according to one's values) (Forgeard, Jayawickreme, Kern, \& Seligman, 2011) within an occupational context (van der Doef and Maes 1999). Past research argues that high workloads erode nurses' well-being because wellbeing is the barometer indicating how well they are coping (Brunetto et al., 2017). Previous research shows that high wellbeing predicts low turnover for public sector Australian nurses (Brunetto, Rodwell, Shacklock, Farr-Wharton, \& Demir, 2016a) and high work harassment is associated with low wellbeing for Australian and Italian public and private sector nurses (Brunetto et al., 2016b).

\section{Turnover Intentions}

Turnover intentions refers to employees' propensity to withdraw from their present workplace and consequently, they conserve their energy at work and redirect it towards looking at alternatives (Van Knippenberg, Van Dick, \& And Tavares, 2007). There are multiple push factors likely to promote turnover intentions, including poor quality relationships and the lack of support given to staff (Van Knippenberg et al, 2007; Lashinger \& Fida, 2014) as well as low wellbeing for US and Australian nurses (Brunetto et al., 2013). In each case, high wellbeing 
was associated with low turnover intentions. The following hypotheses replicate this finding for Bangladeshi nurses.

\section{H3. Employee wellbeing is negatively related to turnover intention}

\section{Mediation and Moderations}

The way that work harassment and wellbeing impact the relationship between LMX and turnover for Bangladeshi public and private sectors is not clear. There are two types of possible effects. First, work harassment moderates the association between LMX and wellbeing, and between LMX and turnover intentions. If this is the case then it means that work harassment affects the direction or strength of the relationship between LMX and wellbeing, and LMX and turnover intentions (Baron \& Kenny, 1986). Second, wellbeing mediates the association between LMX and turnover intentions, which means that employee wellbeing accounts for some of the negative relationship between LMX and turnover intentions. The two types of effects will be discussed further in the following paragraphs.

In terms of COR theory, wellbeing is the measure of an individual's personal resources and is affected by both personal (such as psychological capital) and organisational (such as LMX) factors. In particular, high LMX is associated with high wellbeing for Australian public sector nurses (Brunetto et al., 2016). In addition, high wellbeing is associated with low turnover intentions for Australian and US nurses (Brunetto et al., 2013). This means that if employees have high wellbeing (perhaps because of high personal resources), it may mediate the relationship between LMX and turnover intentions. According to COR theory, if wellbeing is high enough, we expect that the personal resources gained from high wellbeing will mediate the impact of LMX on turnover. The following hypothesis expresses this premise.

\section{H4. Employee wellbeing mediates the relationship between LMX and turnover intention} Moderation refers to a situation where the relationship between two variables depends on a third variable. As stated, previous research shows that high LMX is associated with high 
wellbeing and high work harassment is associated with low wellbeing. In the context of Bangladeshi nurses, we propose that high work harassment results in employees feeling overworked, under-resourced, and that overall their employing organisation's expectations are unrealistic. In such a work environment, even if employees have a good relationship with their manager, it is expected that high work harassment will reduce the positive impact of LMX and employee wellbeing, and decrease the negative impact of LMX on turnover intention. Moreover, we expect that work harassment will moderate the impact of LMX because a manager is considered an agent of the organisation (Eisenhardt, 1989), and thus unrealistic expectations may flow through the manager to the nurses. Therefore, if a nurse thinks expectations are unrealistic, they may associate such expectations with the manager, which when work harassment is high will reduce the positive influence of the manager on employee wellbeing and reduce the negative affect on turnover intentions.

\section{H5. Work harassment moderates the relationship between LMX and wellbeing}

\section{H6. Work harassment moderates the relations between LMX and turnover intentions}

\section{The public-private context}

The context of this research is a comparison of the public and private sector work environment for Bangladeshi nurses. The public sector in Bangladesh is highly bureaucratic and hierarchical, and is described as being over-governed (micro-managed and autocratic) with insufficient or inappropriate governance (subject to corruption) (Ahmed \& Shirahada, 2017). This means that there is a lack of separation between the official practices of public sector officials and personal interests, and a growing realisation that recent reforms, whilst in theory about re-distributing resources from the rich to the poor, did not achieve the stated goals (Khan et al, 2014). Also despite specific legislation promoting equal rights for women, reforms have not made much of an indentation on the glass ceiling imposed upon women by the dominant 
culture in play (Jamil, Aminuzzaman \& Hoque, 2013). According to Ahmad et al. (2007) (cited in Ghuman \& Singh, 2013) reforms were supposed to provide greater access to healthcare for the poor. However, in practice, the rich were given greater access to resources, and nurses were dissuaded against going to poorer regions because of inadequate infrastructure facilities. In summary, reforms did not achieve a greater focus on efficiency or achieving outcomes. Hence, LMX, work harassment, wellbeing and turnover is expected to be worse for public sector nurses. The hypothesis is:

H7: Private sector employees perceive higher LMX and wellbeing and lower work harassment and turnover compared with public sector nurses.

\section{Method}

\section{Sample and procedure}

In the first instance, nurses who had completed an undergraduate and/or postgraduate nursing qualification from the Grameen Caledonian College of Nursing, Dacca, Bangladesh were invited to complete the survey. In total, 191 useable surveys were returned (redemption rate of $62 \%$ ). Additionally, 126 useable surveys were received from nurses working in two public sector hospitals in Dacca, Bangladesh. The data was collected in 2017 and 2018.

In total, 317 useable surveys were obtained, 131 from the public sector and 186 from the private sector. The demographic data depicts that unlike the ageing nursing workforce in many OECD countries (World Health Organization, 2018); the majority of nurses sampled in the Bangladesh public and private sectors were less than 31 years of age. As well, many have less than one-year employment with their current hospital, with the majority having less than 5 years employment in their current hospital. In the public sector, there were 34.4 per cent males $(n=45)$ and 65.6 per cent females $(n=86)$, which is a stark comparison to the private sector with 4.3 per cent males $(n=8)$ and 95.6 per cent females $(n=178)$. 


\section{Measures}

All items for each scale were scored on a six-point Likert scale from 1 (strongly disagree) to 6 (strongly agree). The reliability for each scale is depicted in Table 1 .

Leader-member exchange. To measure a nurse's relationship with their line manager, we used the Leader-Member Exchange (LMX) instrument developed by Graen and Uhl-Bien (1995). An example of an item is 'My manager is satisfied with my work.'

Employee wellbeing. We measured an employee's sense of their wellbeing with the four items from Brunetto, Farr-Wharton, and Shacklock (2011). A sample item includes 'Overall, I am reasonably happy with my work life.’

Turnover intentions. To identify nurse intentions to leave their employing organisation, we used three-items adopted from Meyer, Allen, and Smith (1993) scale of turnover intentions, including, 'I frequently think about leaving my current employer.'

Work harassment. Rayner and Cooper (2006) developed a scale to measure task-attack, personal-attack and intimidation. To measure work harassment, we used three items that examine 'task-directed attack,' including the item '[This hospital] sets unrealistic targets.'

Control variables. Many variables within the nursing and turnover intention literature may impact turnover intentions. We controlled for tenure within a hospital because research has found those with longer tenure have a lower intention to leave their employing organisation (Ng \& Feldman, 2009b). Although there is limited agreement in the literature, we also controlled for gender, with one study suggesting women quit their job less than their male counterparts (e.g., Lyness \& Judiesch, 2001), and a larger scale study of 475,000 professionals and managers concluding women were more likely to voluntarily leave. Finally, we controlled for age because of $\mathrm{Ng}$ and Feldman's (2009a) postulation that older employees typically develop stronger ties to their colleagues and their employing organisations. 


\section{Data analysis}

To test a moderated mediation model, we used SPSS v.25 and the PROCESS Macro by Andrew Hayes (Hayes, 2018). The PROCESS macro is a better alternative to test mediation when compared to approaches such as that prescribed by Baron and Kenny (1986), as there is no requirement for the data to be normally distributed. Within the PROCESS macro, we used model 8 and applied 95 per cent bootstrap confidence intervals across a sample of 5000. To confirm the factor structure and to account for common method variance, we undertook a confirmatory factor analysis of the public and private sectors. To check and control for common method variance, we included a common latent factor (Podsakoff, Mackenzie, Lee, \& Podsakoff, 2003).

\section{Results}

Descriptive statistics and intercorrelations

Table 1 depicts the descriptive statistics, scale reliability and intercorrelations. Notably, LMX did not significantly influence turnover intentions in the public sector. Although not significant, the $p$-value was nearing statistical significance $(p=0.06)$.

Confirmatory factor analysis

The hypothesised measurement model resulted in a poor fitting model for the private $(\mathrm{CMIN} / \mathrm{DF}=2.330, \mathrm{RMSEA}=0.085, \mathrm{CFI}=0.886$ and $\mathrm{SRMR}=0.066)$ and public $(\mathrm{CMIN} / \mathrm{DF}$

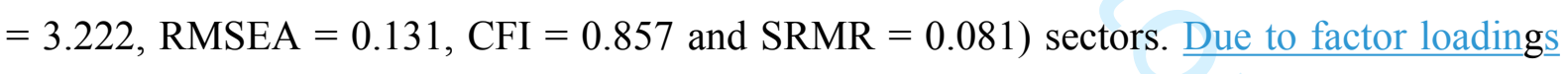
below 0.5 , a number of observed items were removed. We removed three LMX questions: 1. 'Regardless of how much formal authority he/she has built into his/her position, what are the $\underline{\text { chances that your leader would use his/her power to help you solve problems }}$

in your work? (What are the changes that you would)'; 2 . 'have enough confidence in my leader that I would defend and justify his/her decision if he/she were not present to do so?'; and 3. ‘How would you characterize your working relationship with your leader?'. (numbers 4, 6, \& 
7) We also removed one and-wellbeing itemquestion-4, 'I get enough time to reflect on what I do in the workplace'based on factor loadings below 0.5 and high modification indices. The modified measurement model fit the data well for the private $(\mathrm{CMIN} / \mathrm{DF}=1.893 .222, \mathrm{RMSEA}$ $=\underline{.0690 .131}, \mathrm{CFI}=\underline{.9450 .857}$ and $\mathrm{SRMR}=\underline{.0580 .081)}$ and public $(\mathrm{CMIN} / \mathrm{DF}=\underline{1.983} .222$,

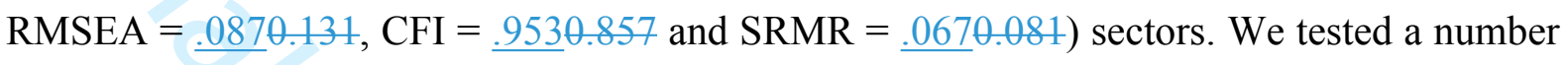
of alternate models to establish the distinctiveness of the variables and the modified measurement model. As depicted in Table 2, the fit of the alternate models was significantly worse when compared with the modified measurement model for the private and public sectors.

The addition of a common latent factor (CLF) to the modified measurement model (model 4) for the private and public sectors resulted in improved model fit. In addition, a chisquare difference test for each sample revealed that the CLF model was statically different from the modified measurement model, and thus common method variance may explain a significant portion of the variance. To identify where common method variance was problematic, we compared the standardised factor loadings of the modified measurement model and the CLF model. To the best knowledge of the authors, there are no published cut-off values for the comparison of standardised factor loadings. We considered any loading difference above 0.20 to be excessively large and retained the CLF control. The final measurement model for the private and public sector include the bias-corrected estimates (i.e., controlling for common method bias). Specifically, to be able to test the hypotheses within the PROCESS macro, we imputed the bias-correct composite variables from AMOS into SPSS.

Testing the hypotheses

Figure 1 depicts the results for hypotheses 1-3 for the public and private sector. LMX predicts turnover $(\mathrm{H} 1)$ for private sector employees only and LMX predicts wellbeing $(\mathrm{H} 2)$ for both public and private sector and wellbeing predicts turnover intentions (H3) for public sector employees only. There was support for the acceptance of hypothesis 4 (mediation), with a 
significant indirect effect $\mathrm{SE}=-.21, \mathrm{CI}(-.42,-.10)$. As mentioned, the direct effect between LMX and turnover intentions was not significant, providing support that wellbeing completely mediates the relationship between LMX and nurse turnover intentions in the Bangladesh public sector. We also found work harassment did not moderate the relationship between LMX and turnover intentions (i.e., rejecting hypothesis 6), but, as depicted in Figure 2, work harassment did moderate the path between LMX and employee wellbeing (i.e., providing support for the acceptance of hypothesis 5). Specifically, high work harassment reduced the influence of LMX on employee wellbeing.

In relation to the private sector, wellbeing did not directly relate to turnover intentions, so we rejected hypothesis 2 . Due to the rejection of hypothesis 3 , and insignificant indirect effects, we rejected hypothesis 4 . Finally, work harassment did not moderate the relationship between LMX and wellbeing, or LMX and turnover intentions. As such, we rejected hypotheses 5 and 6 .

\section{Discussion}

This paper examines the impact of line management on work harassment, wellbeing and turnover of public and private nurses in Bangladesh using a COR framework. One factor which has been identified as having a negative impact, especially in the public sector, is work harassment and in developed countries, the manager either alleviates the situation or makes it worse (Brunetto et al, 2016b). However, research in Brazil found that management did not have the same impact compared with Anglo-American and/or European countries such as UK and Australia (Brunetto et al, 2015), hence, we examined the impact of management on public and private nurses' perceptions of work harassment in Bangladesh. Additionally, since, private sector nurses operate in hospitals that have attempted to implement some typically western HR systems, it seems likely that turnover should be less because the influence of culture (corruption etc) should be less. 
In terms of the first and second hypotheses, previous research had already shown that effective management is associated with high wellbeing and low turnover intentions for public and private sector employees in Anglo-American and European countries (Martin et al., 2016; Brunetto et al., 2018, 2013). The findings from this research support previous research showing that high LMX is associated with high wellbeing for both public and private sector nurses in Bangladesh. The role of the line manager is to act as a source of support when workloads are physically and mentally draining - hence in the ideal situation, they provide a barrier to stress, in turn supporting high wellbeing (Lashinger \& Fida, 2014). However, high LMX is only associated with low turnover for private sector nurses in Bangladesh. One explanation is that public sector nurses are unlikely to have a modern HR system in place (of which a basic manager-employee relationship is an important cornerstone), and instead, the traditional culture may be more important in determining whether employees stay or leave (Ahmed \& Shirahada, 2017; Khan et al., 2014). Khan et al (2014) argues that in Bangladesh, the policy may be similar to a developed country, but the implementation of HR policies has been challenging, especially in the public sector. Hence, it seems likely that family ties and corruption still dominate decision making, in turn negating the role of managers.

Additions, the findings suggest that in contrast to the role of line managers of professionals in Anglo-American and European countries, who play two distinct (sometimescontradictory) roles - professional hybridity, firstly developing nurses' professional skills and knowledge, while still acting as their line manager (Noordegraaf, 2015); the role of public sector line managers in Bangladesh is unclear because nurses' wellbeing is determined by how work harassment mediates the relationship between LMX and wellbeing. One possible reason is that their managers are not engaging in professional activities such as socialising them into the professional norms and values associated with nursing. Khan et al (2014) suggests that in countries like Bangladesh, increased professionalization of the Bangladeshi public sector has 
failed to change 'who gets what' and how workplaces are organised (Jamil, 2013). Hence LMX may not be based on professional factors and the managers may not act as positive antecedents of nurses' wellbeing as is the case in developed countries (Martin et al, 2016). However, further research should examine why a significant relationship was absent.

Regarding hypothesis 3, previous research had already shown that high wellbeing was associated with low turnover intentions for nurses in the USA and Australia (Brunetto et al., 2016). In terms of COR theory, high wellbeing is an important personal resource that employees want to develop, and conserve when required (Hobfoll 2011). The findings from this research are consistent with past research-and COR theory for public sector Bangladeshi nurses only. More research is required to investigate the relationship for private sector nurses. It may be that the traditional culture has a role to play in better understanding the relationship between wellbeing and turnover for Bangladeshi nurses working in the private sector (Khan et al., 2014). Such insight may better explain how employees build wellbeing in the workplace, and importantly, why they stay even in workplace enrivonments that deplete their wellbeing.

The mediation and moderation processes shed further light on understanding the differences in the public and private workplaces for nurses in Bangladesh. Past research shows that work harassment is a product of the era of austerity, which now governs the work lives of many professionals in the caring industry, especially in the public sector in developed countries. However, very little is known about how reforms (if any?) have affected the professional workplace in developing countries. In ideal circumstances, professionals expect their line managers to mediate unrealistic organisational work demands and provide appropriate and sometimes-extra support resources to ensure both task completion and professional development occurs (Farr-Wharton et al., 2011). In this study, work harassment does mediate the relationship between LMX and wellbeing for public sector nurses, but not for private sector 
nurses. Over half of public sector Bangladeshi nurses' wellbeing is explained by how work harassment mediates the relationship between LMX and wellbeing.

While previous research shows that LMX predicts work harassment (Farr-Wharton et al., 2018), and work harassment predicts employees' wellbeing (Xerri et al., 2016), this paper adds new evidence about the role of work harassment in moderating the relationship between management and wellbeing of public sector nurses in Bangladesh. More specifically, in the public sector, when work harassment was low, management had a greater positive influence on employee wellbeing. These results provide new evidence about the influence of work harassment on the well-established association between management practices and wellbeing. This means that the role of the manager is just as important in developing countries such as Bangladesh as it is in developed countries, although culture may affect how it works. It also aligns with COR theory in that those with fewer resources are less able to apply resources to develop other resources, which suggests that those managers who support their nurses impact their decision to stay by improving their wellbeing. However, this is the case for public sector nurses only.

In contrast, LMX explains just under a quarter of private sector nurse wellbeing, and work harassment has no significant impact on this association. Further, there is no evidence of work harassment moderating the relationship between LMX and turnover in this study, although, high LMX, work harassment and wellbeing does explain just under a third of the turnover intentions of public sector Bangladeshi nurses. In the case of private sector Bangladeshi nurses, work harassment alone explains well over a third of their turnover intentions. This suggests that the manager may not have the power to affect how work harassment influences turnover intentions for private sector nurses. This is in contrast to research findings in the Anglo-American and/or European countries which and may suggest a significantly different work context for these private sector nurses working in one of the least 
developed countries in the world. The findings provide a platform for more research about management issues facing public and private professionals such as nurses in developing countries such as Bangladesh.

The last hypothesis examined the differences between the public and private sector nurses' level of satisfaction with LMX, work harassment, wellbeing and turnover. The belief was that private sector nurses would perceive higher LMX and wellbeing and lower work harassment and turnover intentions compared to their private sector counterparts. The hypothesis was based on information that public sector reforms have not been successfully implemented, the public sector in Bangladesh is poorly resourced, and nurses are actively dissuaded from going to poorer regions because of inadequate infrastructure facilities (Jamil, 2013). However, the hypothesis was rejected because, across every factor, public sector nurses were more satisfied with management, had higher wellbeing, perceived lower work harassment and turnover intentions (See Table 1). One explanation using COR theory is that private sector nurses' wellbeing is being conserved and developed from other resources not examined in this paper. Future studies should further examine the antecedents, mediators and moderators relating to work harassment.

In reviewing this study, there are some limitations to consider. First, the cross-sectional data was susceptible to common method bias (Podsakoff et al., 2003). However, as mentioned, a common latent factor was included, and several items that were explained by common method variance were controlled. Nonetheless, future research should examine work harassment over time to improve insight and design relating to common method bias. Second, although a study on Bangladeshi nurses provides some insight into work harassment in developing countries, further research is required about other developing countries. Further, another limitation relates to the sample in that most of the nurses had worked less than five years in their current employment, and therefore, there may be other factors, other than LMX 
and wellbeing affecting their intention to stay that have not been examined in this paper. Finally, the findings are limited by the sample size for each sector, and the type of demographic information collected that could have assisted in explaining it. Further research should be undertaken to increase the sample size, which will improve the stability of the results, and provide enough statistical power to examine more complex research models, including a thourough examination of work harassment antecedents, mediators and moderators.

\section{Conclusion}

The findings suggest that work harassment is prevalent in the Bangladesh public and private nursing profession. This study used a COR theoretical framework to compare the impact of management practices (LMX) and work harassment on the wellbeing and turnover intentions of Bangladeshi public and private nurses. Using COR theory, we found that LMX is a positive resource caravan passageway that can build wellbeing for public sector employees; however, the association was not significant for private sector nurses. Hence, COR theory principle 2 offers one explanation of is relevant for public sector employees' behaviour in that it assumes thatbecause the manager provided enough of a 'cushion' for them to form a barrier against the negative influence of work harassment on their wellbeing (Hobfoll, 2001). Similar to per past

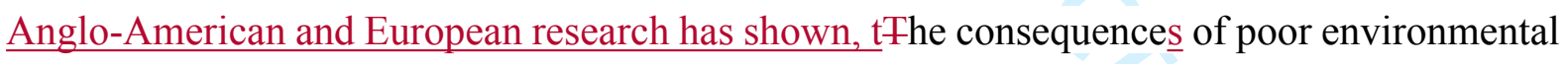
conditions, such as work harassment, is that it does affect how public sector nurses in Bangladesh are able to take advantage of the relationship with their manager. However, there was no evidence that employees who perceive high support from their line managers are less likely to be negatively influenced by work harassment (Hobfoll, 2011). The theory was useful for examining how work harassment affects public and private sector nurses differently. Therefore, whilst COR theory was somewhat useful in explain some behaviour of public sector Bangladeshi nurses, it was far less relevant for explaining the behaviour of those in the private 
sector. This is an important finding because theories and concepts developed and validated in

Anglo-American contexts assume underlying cultural similarities that may not be accurate in developing countries. Future research should perhaps test other well established theories (and concepts) across multiple contexts to verify their validity.

The implication of this research relates directly to public sector management. For example, the impact of management is different in Bangladesh compared with past AngloAmerican and European studies, and one factor is likely to be the traditional cultural stronghold, especially the impact of ties and corruption, which affects nurses' turnover intention, especially in public sector nurses areas (Ahmed \& Shirahada, 2017). Future research should explore those assertions. Additionally, there are significant differences in how public and private sector nurses respond to, and cope with work harassment. Hence, simply transplanting management models from developed countries for managing healthcare in developing countries may not yield its intended benefits. More research is required to better understand how managers affect how professionals work in developing countries. 


\section{References}

Ahmed, T \& Shirahada, K (2017) Toward a sustainable healthcare service system in a limited resource context: Case study of Bangladesh rural advancement committee's healthcare system, International Journal of Healthcare Management,

DOI: $10.1080 / 20479700.2017 .1389512$

Baron, R. M., \& Kenny, D. A. (1986). The moderator-mediator variable distinction in social psychological research: Conceptual, strategic, and statistical considerations. Journal of Personality and Social Psychology, 51(6), 1173-1182.

Branch, S., Ramsay, S., \& Barker, M. (2013). Workplace bullying, mobbing and general harassment: A review. International Journal of Management Reviews, 15, 280-299

Brunetto, Y., Farr-Wharton, R., \& Shacklock, K. (2011). Using the Harvard HRM model to conceptualise the impact of changes to supervision upon HRM outcomes for different types of public sector employees International Journal of Human Resource Management 22(3), 553-573.

Brunetto, Y., Rodwell, J., Shacklock, K., Farr-Wharton, R., \& Demir, D. (2016a). The impact of individual and organizational resources on nurse outcomes and intent to quit. Journal of advanced nursing, 72(12), 3093-3103.

Brunetto, Y., Xerri, M., Shriberg, A., Farr-Wharton, R., Shacklock, K., Newman, S., \& Dienger, J. (2013). The impact of workplace relationships upon engagement, wellbeing, commitment and turnover for nurses in Australia and USA. Journal of advanced nursing, 69(12), 27862799.

Brunetto, Y., Xerri, M., Trinchero, E., Beattie, R., Shacklock, K., Farr-Wharton, R., \& Borgonovi, E. (2018). Comparing the impact of management on public and private sector nurses in the UK, Italy, and Australia. Public Management Review, 20(4), 525-544.

Brunetto, Y., Xerri, M., Trinchero, E., Farr-Wharton, R., Shacklock, K., \& Borgonovi, E. (2016b). Public-Private Sector Comparisons Of Nurses' Work Harassment Using Set: Italy and Australia. Public Management Review, 18(10), 1479-1503. doi:DOI:10.1080/14719037.2015.1114136

Brunetto, Y., Shacklock, K., Teo, S., Farr-Wharton, R., \& Nelson, S. (2015). Nurses' managers, learning options and organisational commitment: Australia, Brazil and England. Journal of Nursing Management. $23(8), 1029-1038$

Dick, G. P. (2010). Can the organisation and supervision environment influence both bullying and organisational commitment? Evidence from a police force survey. Kent University Working Paper Series 196.

Diefenbach, T. (2009). New public management in public sector organizations: the dark sides of managerialistic 'enlightenment'. Public Administration, 87(4), 892-909.

Einarsen, S. (2000). Harassment and bullying at work: A review of the scandinavian approach. Aggression and Violent Behavior, 5(4): 379-401

Einarsen, S., Hoel, H., \& D. Zapf, D. eds. (2011). Bullying and Harassment in the Workplace: Developments in Theory, Research and Practice ( $2^{\text {nd }}$ Edition). CRC Press, Taylor \& Francis Group, Boca Raton, Florida.

Einarsen, S., \& Nielsen, M. (2015). Workplace bullying as an antecedent of mental health problems: A five-year prospective and representative study. International Archives of Occupational and Environmental Health. 88: 131-142

Eisenhardt, K. M. (1989). Agency theory: An assessment and review. Academy of Management Review, 14(1), 57-74.

Farr-Wharton, R., Brunetto, Y., \& Shacklock, K. (2011). Professionals' manager-subordinate relationships, autonomy and commitment in Australia: a leader-member exchange theory perspective. The International Journal of Human Resource Management, 22(17), 3496-3512. 
Forgeard, M. J., Jayawickreme, E., Kern, M. L., \& Seligman, M. E. (2011). Doing the right thing: Measuring wellbeing for public policy. International journal of wellbeing, 1(1), 79-106.

Ghuman, B. S., \& Singh, R. (2013). Decentralization and delivery of public services in Asia. Policy and Society, 32, 7-21

Graen, G., \& Uhl-Bien, M. (1995). Relationship-based approach to leadership: Development of leader-member exchange (LMX) theory of theory over 25 years: Applying a multi-level multidomain perspective. Leadership Quarterly, 6(2), 219-247.

Halbesleben, J. R., Neveu, J.-P., Paustian-Underdahl, S. C., \& Westman, M. (2014). Getting to the "COR" understanding the role of resources in conservation of resources theory. Journal of management, 40(5), 1334-1364.

Hayes, A. F. (2018). Introduction to mediation, moderation, and conditional process analysis: A regression-based approach. New York: Guilford Press.

Hobfoll, S. E. (1989). Conservation of resources: A new attempt at conceptualizing stress. American psychologist, 44(3), 513-524.

Hobfoll, S. E. (2001). The influence of culture, community, and the nested-self in the stress process: advancing conservation of resources theory. Applied psychology, 50(3), 337-421.

Hobfoll, S. E. (2011). Conservation of resource caravans and engaged settings. Journal of occupational and organizational psychology, 84(1), 116-122.

Jamil, I., Aminuzzaman, S., \& Haque, S. (2013) Introduction: Governance in South, Southeast, and East Asia Public Organization Review 13 (4) 341- 347

Khan., R, Miah., M, \& Manzoor., A (2014). Human Resource Management Practices: A Case Study of South Asian Countries IBT Journal of Business Studies 9(1), 83-101

Lyness, K. S., \& Judiesch, M. K. (2001). Are female managers quitters? The relationships of gender, promotions, and family leaves of absence to voluntary turnover. Journal of applied Psychology, 86(6), 1167-1178.

Mahdy, H. A. (2009) Reforming the Bangladesh healthcare system, International Journal of Health Care Quality Assurance, 22(4), 411-416.

Martin, R., Thomas, G., Guillaume, Y., Lee, A. \& Epitropaki, O. (2016). Leader-member Exchange

(LMX) and Performance: A Meta-analytic Review. Personnel Psychology, 69, 67-121.

Meyer, J. P., Allen, N., \& Smith, C. A. (1993). Commitment to organizations and occupations: extension and test of a three-component conceptualization. Journal of applied Psychology, 78(4), 538-551.

$\mathrm{Ng}$, T. W., \& Feldman, D. C. (2009a). Age, work experience, and the psychological contract. Journal of Organizational Behavior, 30(8), 1053-1075.

$\mathrm{Ng}, \mathrm{T}$. W., \& Feldman, D. C. (2009b). Re-examining the relationship between age and voluntary turnover. Journal of Vocational Behavior, 74(3), 283-294.

Noordegraaf, M. (2015) Hybrid Professionalism and Beyond: (New) Forms of Public Professionalism in Changing Organizational and Societal Contexts, Journal of Professions and Organization, 2, 187-206.

Podsakoff, P. M., MacKenzie, S. B., Lee, J.-Y., \& Podsakoff, N. P. (2003). Common method biases in behavioral research: a critical review of the literature and recommended remedies. Journal of applied Psychology, 88(5), 879-903.

Rayner, C., \& Cooper, C. L. (2006). Workplace bullying. In K. E. Kelloway, J. Barling, \& J. J. H. Jr. (Eds.), Handbook of workplace violence. Thousand Oaks: Sage Publications.

Laschinger, H.K.S., Fida, R., (2014). New nurses' burnout and workplace wellbeing: the influence of authentic leadership and psychological capital. Burnout Research 1 (1), 19-28

Seligman, M.E., (2012). Flourish: A visionary new understanding of happiness and well-being. New York: Simon and Schuster.

Tummers, L., Vermeeren, B., Steijn, B., \& Bekkers, V. (2012). Public professionals and policy implementation: Conceptualizing and measuring three types of role conflicts. Public Management Review, 14(8), 1041-1059. 
Tummers, L., Brunetto, Y., Teo, S., (2016) Workplace aggression: Introduction to the special issue and future research directions for scholars, International Journal of Public Sector Management, 29 (1), $2-10$

Van Knippenberg, D., Van Dick, R., \& and Tavares, S. (2007). Social Identity and Social Exchange: Identification, Support, and Withdrawal from the Job. Journal of Applied Social Psychology, 37(3), 457-477.

Van der Doef, M. and Maes, S., 1999. The job demand-control (-support) model and psychological well-being: a review of 20 years of empirical research. Work \& stress, 13(2), pp.87-114.

World Bank. (2016). Overview of Bangladesh. Retrieved from http://www.worldbank.org/en/country/bangladesh/overview

World Health Organization. (2016). World Health Statistics 2016: Monitoring Health for the Sustainable Development Goals (SDGs). World Health Organization. Retrieved from http://www.who.int/gho/publications/world health statistics/2016/en/

World Health Organization. (2018). Nursing and Midwifery Fact Sheet February 2018. Retrieved from http://www.who.int/mediacentre/factsheets/nursing-midwifery/en/

Xerri, M., Farr-Wharton, R., Brunetto, Y., \& Lambries, D. (2016). Work harassment and local government employees: Australia and USA. International Journal of Public Sector Management, 29(1), 54-71.

Zapf, D., Escartin,J., Einarsen, S., Hoel, H. \& Vartia, M. (2011). Empirical findings on the prevalence and risk groups of bullying in the workplace. In Bullying in the Workplace; Developments in Theory, Research and Practice edited by Einarsen, S., Hoel, H., Zapf, D., Cooper. C. CRC press, Taylor \& Francis group, Boca Rata 
Table 1. Correlation matrix

\begin{tabular}{|c|c|c|c|c|c|c|c|c|c|c|c|}
\hline & $\begin{array}{l}\text { Private } \\
\mathrm{M}(\mathrm{SD})\end{array}$ & $\begin{array}{l}\text { Public } \quad \text { C.R } \\
\mathrm{M}(\mathrm{SD})\end{array}$ & $\underline{\mathrm{AVI}}$ & $\underline{\sqrt{A V E}}$ & 1 & 2 & 3 & 4 & 5 & 6 & 7 \\
\hline 1. Gender & - & - & & & 1 & .09 & $.22^{*}$ & -.10 & $.22^{*}$ & -.06 & $.32 *$ \\
\hline 2. Age group & - & - & & & -.07 & 1 & $.20^{*}$ & $.28 * *$ & -.13 & $.18^{*}$ & -.06 \\
\hline 3. Hospital tenure & - & - & & & .04 & $.36^{* *}$ & 1 & -.08 & -.07 & .03 & .04 \\
\hline $\begin{array}{l}\text { 4. Turnover } \\
\text { intentions }\end{array}$ & $2.86(1.32)$ & $1.64(.84) \quad .87$ & .70 & .84 & .09 & $-.26 * *$ & $-.19 *$ & * 1 & $-.29 * *$ & $.50 * *$ & -.15 \\
\hline 5. Wellbeing & $3.90(.50)$ & $4.41(.94) . .93$ & .81 & .90 & .05 & -.00 & -.05 & -.08 & 1 & -.14 & $.68 *$ \\
\hline $\begin{array}{l}\text { 6. Work } \\
\text { harassment }\end{array}$ & $2.74(1.30)$ & $2.61(1.17) .87$ & .69 & .83 & .07 & -.13 & -.07 & $.81 * *$ & -.13 & 1 & -.21 \\
\hline $\begin{array}{l}\text { 7. Leader-member } \\
\text { exchange }\end{array}$ & $3.30(.65)$ & $4.63(1.08) .88$ & .64 & .80 & -.00 & .07 & -.05 & $-.38 * *$ & $.52 * *$ & $-.37 * *$ & 1 \\
\hline $\begin{array}{l}* \text {. Correlation is sigr } \\
0.01 \text { level }(2 \text {-tailed). } \\
\text { average variance ext } \\
\text { scale where } 1=\text { Stron } \\
5=\text { Agree and } 6=\text { Strol }\end{array}$ & $\begin{array}{l}\text { nificant at } t \\
\text { Private sec } \\
\text { racted; } \sqrt{\mathrm{A}} \\
\text { gly Disagre }\end{array}$ & $\begin{array}{l}\text { the } 0.05 \text { leve } \\
\text { ctor below th } \\
\text { VE = square } \\
\text { ee, } 2=\text { Disagr }\end{array}$ & on & $\begin{array}{l}* * . C \\
\text { C.R }= \\
\text { ewVE. } \\
\text { ewhat I }\end{array}$ & $\begin{array}{l}\mathrm{rr} \\
\mathrm{co}\end{array}$ & $\begin{array}{l}\text { ation } \\
\text { posit } \\
\text { All m } \\
\text { ree, } 4\end{array}$ & ig1 & $\begin{array}{l}\text { ificant } \\
\text { ility; } \\
\text { e base } \\
\text { what }\end{array}$ & $\begin{array}{l}\text { the } \\
\mathrm{VE}= \\
\text { on a } \\
\text { gree, }\end{array}$ & & \\
\hline
\end{tabular}

Table 2. Comparison of alternate measurement models

\begin{tabular}{|c|c|c|c|c|c|c|c|}
\hline Model & & $\chi^{2}$ & $\Delta \chi^{2}$ & $\underline{\mathrm{dff}}$ & $\Delta d \mathbf{f f}(\mathrm{p})$ & CFI & SRMR \\
\hline & $\begin{array}{l}\text { Hypothesised measurement model } \\
\text { Private sector }\end{array}$ & 265.217 & & $\underline{113}$ & & .886 & .066 \\
\hline Base & $\begin{array}{l}\text { Modified measurement model - private } \\
\text { sector }\end{array}$ & 111.608 & & $\underline{59}$ & & .945 & .058 \\
\hline 1 & Combine LMX and wellbeing & 181.715 & 70 & $\underline{62}$ & $\underline{3(p<.001)}$ & .874 & .084 \\
\hline 2 & Combine LMX and Harassment & 317.966 & $\overline{206.36}$ & $\overline{62}$ & $3(\mathrm{p}<.001)$ & .731 & .146 \\
\hline 3 & Combine wellbeing and harassment & 229.781 & & $\underline{62}$ & $3(\mathrm{p}<.001)$ & .824 & .118 \\
\hline \multirow[t]{2}{*}{4} & Add CLF to Base model & 76.394 & 35 . & $\underline{46}$ & $13(\mathrm{p}<.001)$ & .968 & .045 \\
\hline & $\begin{array}{l}\text { Hypothesised measurement model (four } \\
\text { factor model) - Public sector }\end{array}$ & 364.098 & & $\overline{11}$ & & .857 & .081 \\
\hline Base & $\begin{array}{l}\text { Modified measurement model - public } \\
\text { sector }\end{array}$ & 116.799 & & & & .953 & .067 \\
\hline 1 & Combine LMX and wellbeing & 309.179 & 192.50 & $\underline{62}$ & $3(\mathrm{p}<.001)$ & .797 & .090 \\
\hline 2 & Combine LMX and Harassment & 326.026 & $\underline{209.23}$ & $\underline{62}$ & $3(\mathrm{p}<.001)$ & .783 & .161 \\
\hline 3 & Combine wellbeing and harassment & 332.380 & 215.58 & $\overline{62}$ & $3(\mathrm{p}<.001)$ & .778 & .164 \\
\hline 4 & Add CLF to Base model & 69.596 & $\underline{42.203}$ & $\underline{46}$ & $13(p<.001)$ & .981 & .046 \\
\hline
\end{tabular}




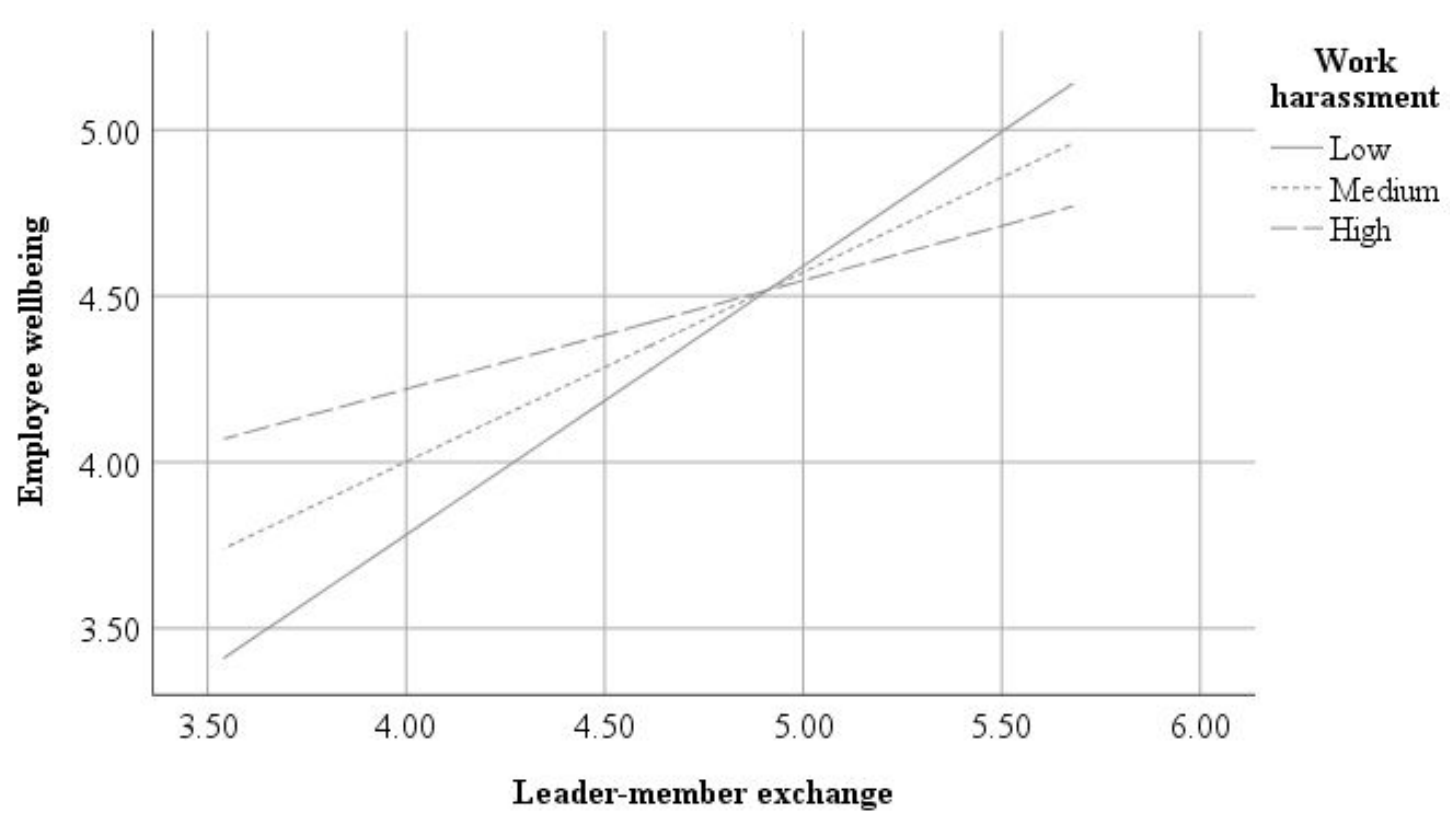

Figure 2. Moderation results - work harassment moderates the relationship between LMX and employee wellbeing. Note: Work harassment standardised for moderation analysis 


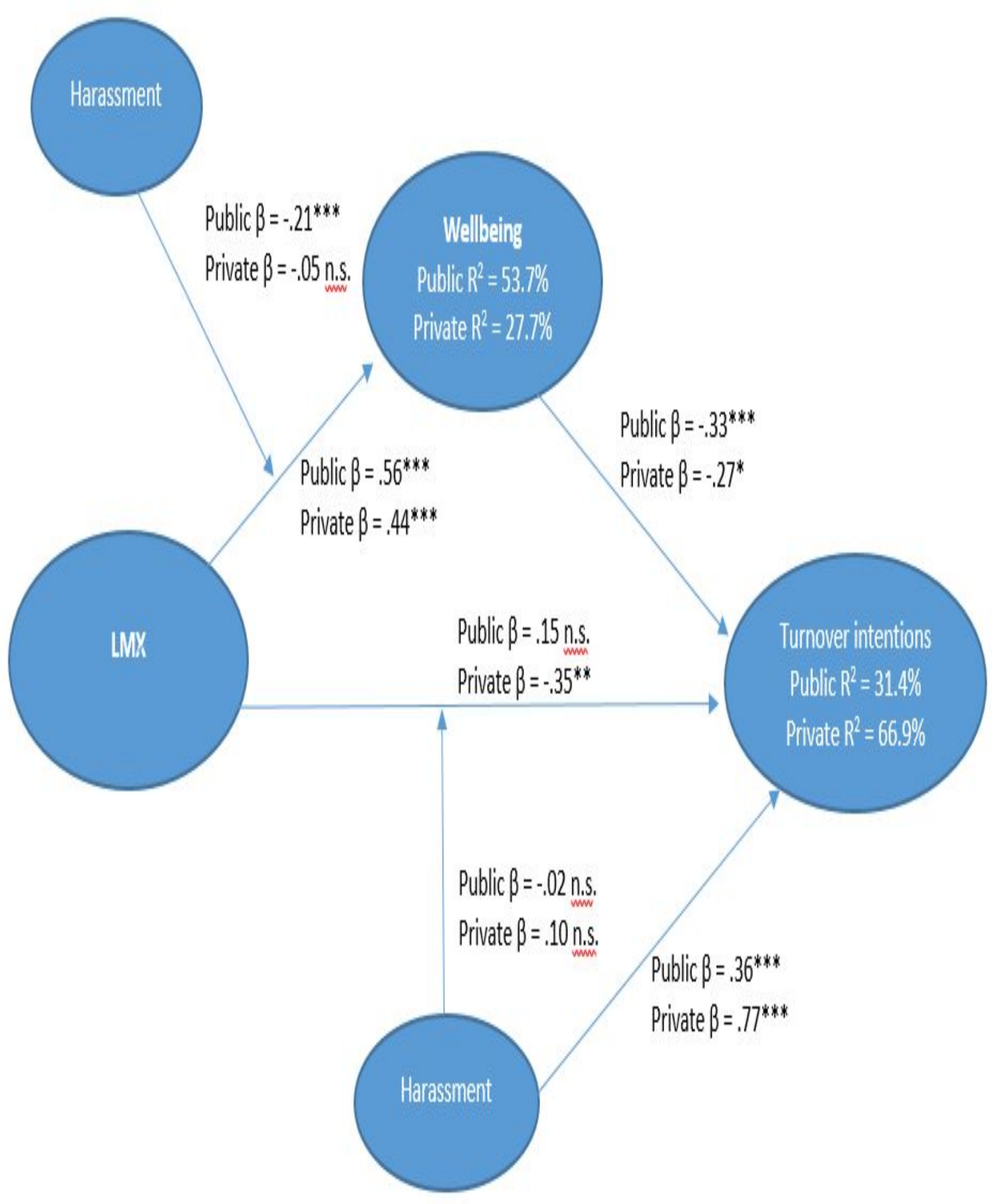




\title{
Comparing the impact of management on public and private nurses in Bangladesh
}

\begin{abstract}
Purpose: This paper uses theory as a lens for comparing the impact of line management on Bangladeshi public and private nurses' perception of work harassment, wellbeing and turnover intentions where Anglo-American and European management models have been superimposed on an existing different culture.
\end{abstract}

Design: Survey data was collected from 317 Bangladeshi nurses' (131 from the public sector and 186 from the private sector). Structural Equation Modelling was used for analysis.

Findings: High work harassment was associated with low wellbeing and management practices explained approximately a quarter of private sector nurse's wellbeing. . In total, management, work harassment and wellbeing explained approximately a third of the turnover intentions of public sector nurses, whereas only work harassment explained approximately a third of private sector nurses' turnover intentions.

Limitation; Cross-sectional data is susceptible to common method bias. A common latent factor was included, and several items that were explained by common method variance were controlled. Further, the findings are limited by the sample size from one sector and the use of only one developing country.

Implications: It is a waste of resources to transplant Anglo-American and European management models to developing countries without understanding the impact on nurses' outcomes.

Originality: Anglo-American and European management models and theories are not easily transferable to the public sector Bangladesh context probably because of the impact of ties and 
corruption. Line management is a positive resource that can build wellbeing for public sector employees only.

Keywords: public-private sector, line management, well-being, work harassment, turnover intentions 


\section{Comparing the impact of line management on public and private nurses in Bangladesh}

Public sector management models do not easily translate to best practice in developing countries, mainly because the conditions are too different. For example, in contrast to developed countries, there are more doctors than nurses in Bangladesh and the demand for nurses' services exceeds supply whilst continuing to grow in line with population growth: hence they work in high-pressure environments (World Health Organization, 2018; World Bank, 2016). As a consequence, this paper examines whether Bangladeshi nurses perceive work harassment similarly to other public and private nurses in developed countries (Brunetto, Xerri, Trinchero, Farr-Wharton, Shacklock, Borgonovi, 2016) and whether this affects their turnover intention. Dick (2010) refers to work harassment as a form of workplace bullying but suggests that it differs from bullying. Specifically, an employee is exposed to work harassment when their employing organisation unreasonably monitors their actions, sets excessively high workloads, and has stringent accountability standards (Brunetto et al, 2016b). As such, the organisation and its managers are the instigators of work harassment.

Work harassment has been identified as a growing problem for public sector managers in developed countries (Tummers, Brunetto \& Teo 2016). For example, Brunetto et al. (2016b) compared work harassment of nurses in Australia and Italy and found significant parallels in their perceptions. More recent research identified a growing presence and acceptance of work harassment in US and UK hospitals (Farr-Wharton, Brunetto \& Xerri, 2019). The delivery of health services has been significantly affected by the cost-constraint that mandated the depth and breadth of reforms over the past four decades which has resulted in the expectation that employees will 'do more with less' (Diefenbach, 2009). However, the situation is even more pronounced in Bangladesh, where the shortages in nurses, especially in the public sector and in particular, in rural areas, is a growing problem (Ahmed \& Shirahada, 2017). Specifically, there are high levels of resource inadequacy, with widespread shortages in basic nursing 
equipment and a lack of an effective nurse management systems (World Health Organization, 2016). Such inadequacies in the Bangladesh system are likely to create a potential haven for work harassment.

The shortage of public sector nurses in Bangladesh is increasingly dire, and in some cases, the families of patients have to act as carers (Mahdy, 2009). If work harassment is not managed effectively, then it may negatively influence the profession's ability to attract new nurses, and retain them in the job. Whilst there is evidence of work harassment across different public sector occupations, such as local government and healthcare workers across different countries - Australia, Italy, USA (Tummers et al 2016; Xerri et al, 2016), there is no evidence as to whether work harassment is an issue in developing countries. In this study, we have two core aims. The first aim is to develop insight into the impact of line management on outcomes such as work harassment, wellbeing and turnover intentions for nurses working in Bangladeshi hospitals. The second aimis to develop a deeper understanding of the similarities and differences across the public and private sectors.

In relation to work harassment, in developed countries the theory argues that the role of the line manager for professionals is very important because of the dual role of the professional mentor and line manager within the organisational hierarchy. However, line managers of professionals are also increasingly faced with the issue of 'professional hybridity' (the sometimes-contradictory expectations that managers will both develop nurses' professional skills and knowledge and ensure task completion) (Noordegraaf, 2015). Never-the-less, a good line manager is one who can mediate the excessive demands of the hierarchy to ensure appropriate resourcing and support is available in relation to task completion and professional development (Farr-Wharton, Brunetto, \& Shacklock, 2011). Although past evidence has established the association between line management practices and wellbeing, and between line management practices and turnover intentions (Brunetto et al., 2013), to the best 
knowledge of the authors, there is no evidence about how work harassment may change these established relationships. As such, we aim to add to the existing evidence by examining the role of work harassment in affecting the association between satisfaction with line management and employee wellbeing, and in turn, turnover intentions within a developing country such as Bangladesh. Such evidence adds to the literature about the impact of line management on employee wellbeing and turnover intentions of nurses within developing countries, in this case, Bangladesh.

Managing both public and private sector employees in developing countries can be challenging as international HR systems are imported into existing culture-driven decisionmaking systems. Most managers were traditionally autocratic in their practices in South Asia (Khan, Miah \& Manzoor, 2014). However, in the last three decades, the service sector has undergone significant change mainly because of global forces and government reforms that have elevated the importance of human resources slowly, especially in terms of the significance of ensuring adequate qualifications and competencies for key positions. This change has led to the introduction of modern HRM and strategic systems, however, the new systems have not replaced the previous stronghold of culture in organisational decision-making. This means that family ties and corruption still dominate decision making, favouring some and disadvantaging the majority, and whilst those with strong international connections are likely to have basic HR policies and structures evident in countries such as the USA and UK, the difference is in their ability to implement those policies into practice (Khan et al., 2014, p 99). Hence, while Bangladeshi organisations may have specific policies about work hours and duties, in practice, there is probably greater scope for work harassment in Bangladesh compared with AngloAmerican countries such as the UK, USA and Australia.

This paper compares the relationship between line management, work harassment, wellbeing and turnover intentions of public and private sector nurses using a Conservation of 
Resources (COR) theoretical framework (Hobfoll, 1989). COR theory is typical of theories originally developed and tested within an Anglo-American and/or European context (history, culture) to explain employee outcomes regarding their ability to maintain and grow their personal resources such as health and wellbeing. However, it may not have as much relevance in explaining behaviour in developing countries. COR theorists argue that when the organisational environment erodes their personal resources through work harassment, their wellbeing is negatively impacted and this motivates them to protect their well-being by regulating their work practices. COR theorists argue that if extra support (e.g., from managers) is not available, then employees may offset perceived resource losses, and potential shortages, by reducing effort at work or leaving the organisation (Hobfoll, 2011). The research questions (RQs) guiding the study are:

$R Q$ 1: What is the impact of line management on work harassment, wellbeing and turnover of public and private sector Bangladeshi nurses?

$R Q$ 2: What are similarities and differences in the relationship between line management, work harassment, wellbeing and turnover for nurses in the public and private sector?

The next part details a relevant review of the literature and identifies hypotheses that reflect the gap in the literature.

\section{Background}

\section{Conversation of Resources (COR) theory}

The COR theory is ideal for understanding employee outcomes, especially in the face of resource adversity (Hobfoll 1989). The way COR theory works is via two principles and four Corollaries (Hobfoll, 2011). Principle one: a loss of resources likely has a bigger effect than acquiring new resources. Principle two: to gain new or more resources, employees must firstly spend time or energy (resources) in those activities likely to lead to more resources. Further, if employees believe they are likely to experience a loss of resources (such as a loss 
of wellbeing) they will likely engage in activities (sick leave/ look for another job) to protect themselves against falling wellbeing. The four corollaries comprise four assumptions Firstly, the higher employees' perception of resources are, the easier it is for them to gain resources, whereas employees with fewer resources are more susceptible to resource loss, and less able to gain resources. Secondly, it is likely that an initial loss of resources will lead to further losses; whereas it is likely that a resource gain will likely lead to further resources gains. Fourthly,) employees are likely to act defensively as a strategy to prevent further loss of resources (Halbesleben, Neveu, Paustian-Underdahl, \& Westman, 2014).

Drawing from COR theory, each organisation makes decisions that produce either positive or negative environmental conditions, called 'resource caravan passageways' (Hobfoll (2011). Under ideal conditions, an employee can build resources such as increased wellbeing by investing other resources at their disposal; in this case, effective supervision is a social resource (caravan passageway) an employee can draw upon. Whereas, if the resource caravan passageway is high levels of work harassment, employees have low resources and are thus less able to orchestrate resource gain. In this case, we propose that work harassment may result in employees that are less able to develop wellbeing with the social resource derived from the relationship with their manager.

Using COR theory to explain the relationship between management, work harassment, wellbeing and turnover, we argue that effective management practices is a positive resource caravan passageway that can build wellbeing, whereas work harassment is a negative resource caravan passageway that will influence an employee's ability to develop resources such as wellbeing. The consequences of the poor environmental conditions are likely to lead to increased turnover (Hobfoll, 2001). Using COR corollary 4, the issue will be whether the support from the manager is enough of a 'cushion' to form a barrier against the negative influence of work harassment on wellbeing, and the flow on to an intention to turnover. As per 
corollary 4 , those with a lack of resources are likely to act defensively to conserve against further resource loss. That is, those who lack resources are unable to apply resources to fight against resource loss (Hobfoll, 2011), and thus to conserve further loss, they may choose to eject themselves from the situation by intending to leave their employing organisation. In contrast, those employee with adequate resources are likely to be able to invest them in resource develop, which is likely to be evident by high wellbeing and low turnover intentions.

\section{Variables under examination}

\section{Work Harassment (resource caravan passageway)}

The term 'work harassment' is challenged by different groups of researchers and the same work has multiple meanings depending on the country. Originally, the term emerged from 'harassment' meaning “... all repeated behaviors [sic] which ... provoke, frighten, intimidate, or bring discomfort to the victim" (Brodsky (1976) cited in Einarsen, 2000; p382). However, the definition has moved on in different ways in different countries. For example, the Scandinavian researchers adopted different terms compared with the Anglo-American researchers for similar behavioural aggression and their remains no consensus about definitions for the different negative work behaviours (Branch, Ramsay, \& Barker, 2013; Einarsen, \& Nielsen, 2015). In terms of public sector HR in Anglo-American and/or European countries, work harassment has emerged as a negative work behaviour, similar to bullying or mobbing because it is characterised by repeated ill-treatment with negative consequences for the individual (Zapf, Escartin, \& Einarsen, 2011).

Originally, work harassment was conceptualised as part of bullying because of its negative impact on the individual (Raynor, 2002). More recently, work harassment is differentiated from bullying for three main reasons. Firstly, bullying is directed at an individual, whereas work harassment is about task completion and affects large numbers of front-line employees facing a chronic problem of demand outstripping supply, forcing them either to 
choose 'who get what' (or who misses out) and/or 'to do more with less' on a continual basis (Dick, 2010; Xerri, Farr-Wharton, Brunetto, \& Lambries, 2016). Secondly, bullying can involve physical or mental abuse directed at an individual, whereas work harassment is not personal. Instead, it is the result of a resourcing decision made originally by governments, and then by senior management in allocating resources to each department (Tummers, et al, 2016).

Thirdly, the incidence of work harassment relies on the power of professional principles to ensure patients/clients/customers are serviced, irrespective of organisational resourcing constraints. This is because universities and the professional bodies regulate entry into the professions and work practices are underpinned by specific norms and values (such as professional standards of service and effectiveness) (Farr-Wharton et al., 2011). This in turnoften brings them into conflict with organisational norms (task completion/ reduced per capita costs) leading to 'professional hybridity' (Noordegraaf, 2015). One of the outcomes for healthcare professionals is increasing stress from role conflict as professionals work overtime (often against organisational directions) (Tummers, Vermeeren, Steijn, \& Bekkers, 2012). Hence, work harassment is a type of negative workplace behaviour that is emerging as a growing HR problem, especially for healthcare professionals working in the public sector where austerity dominates resourcing decisions. In particular, Tummers et al. (2016) identified the rise in stress (and stress-related illnesses) emerging amongst professionals because of increasing work harassment, role overload and role ambiguity.

For healthcare workers, high demand is a worldwide phenomenon. In those countries where there is also a shortage of nurses, the workplace conditions are even more likely to enhance, rather than detract from work harassment. For example, Laschinger and Fida (2014) found that in Canada, even new graduate nurses were reporting high-stress levels leading to falling wellbeing and the worst cases, a steady rise in the incidence of high levels of burnout and turnover. Laschinger et al. $(2014$, p19) argue that the key contributors are heavy workloads 
and inadequate staffing that in turn, create the negative environmental conditions ripe for increasing job dissatisfaction, and:

"Similar to other helping professions, the prevalence of burnout in nursing is particularly high, because of the high emotional and physical demands of this work"

However, it may be the impact of cultural factors intervening for Bangladeshi nurses that negates the impact of work harassment if for example, they perceive the importance of helping pateints are over-riding the impact of high workloads on their wellbeing. This is what is unknown - do Bangladeshi employees such as nurses behave similarly to employees in developed countries? In terms of COR theory, high work harassment acts as a negative work behaviour that erodes personal resources. If an employee has a shortage of resources - they will likely see a reduction in their wellbeing. As such, principle two is enacted and consequently, employees seek to control the negative flow of wellbeing by looking for another job (as one example of acting defensively) (Hobfoll 2011). Unless there is a counterbalance of positive resources, for example - positive relationships with their managers that can buffer and negate the impact of work harassment, then employees' personal resources will be negatively affected.

\section{Employee Resources (resource caravans)}

\section{Manager Support - Leader-member exchange (a social support resource)}

Manager-subordinate relationships can be conceptualised via the LeaderMember Exchange (LMX) framework. LMX is a Social exchange theory (SET) variable because the potential benefits from effective workplace relationships far outweigh the initial outlay of good will. LMX argues that managers form different quality relationships with employees: for some their workplace interactions are meaningful and over time leads to mutual reciprocity (insiders). On the other hand, for others the interactions are negative and 
consequently a relationship forms that is built only on the work contract specifications (Martin, Thomas, Guillaume, Lee, \& Epitropaki, 2016). The benefits of an effective LMX relationship were identified in a meta-analysis by Martin et al. (2016) as high role performance and high citizenship behaviour as well as low counteractive workplace behaviour. This relationship becomes even more important when it involves professionals because there is an added responsibility on the part of the manager to ensure the employee is socialised into the values, norms and practices associated with the profession and adheres to professional standards (Brunetto et al., 2018). However, research about public sector nurses in Brazil found that that the theories and "truths" accepted as the behaviour of employees such as nurses in developed countries does not always translate to the behaviour of nurses in BRIC countries. For example, Brunetto, Shacklock, Teo, Farr-Wharton \& Nelson (2015) found that managers had minimal impact on Brazilian nurses' behaviour for numerous reasons, including that there were different contracts in place with some nurses reporting to managers in the capital of Brazil, not the hospital, and the impact of corruption on nurses' actions in the workplace.

However, using COR theory, when LMX is high, it suggests that employees can access support from managers or someone in management. Hence, it is not surprising that previous research in developed countries suggests that high LMX is associated with high wellbeing and low turnover for nurses in Anglo-American and European countries such as Australia, Italy and the UK (Brunetto et al., 2018) as well as the USA (Brunetto et al., 2013). The reason as to why LMX is associated with high employee performance is because as Lashinger and Fida (2014) found in their research of Canadian nurses, high LMX indicates that nurses are receiving high levels of support from managers, which in turn provides a barrier to stress and helps maintain high employee wellbeing. However, the relationship has not been examined for public and private sector Bangladeshi nurses, where cultural influences on HR and workplace relationships do not necessarily follow the Anglo-American and/or European model. To test 
whether similar relationships are in play with Bangladesh for nurses, the following hypotheses are used.

\section{H1. LMX is negatively related to turnover intention \\ H2. LMX is positively related to employee wellbeing}

\section{Employee well-being (an individual/personal resource)}

'Well-being' is a general term with multiple definitions across different disciplines and there is no agreement on a definition except; however, that it is a multi-faceted concept (incorporating physical, job-related, psychological, social wellbeing), and each time it is used, it requires explicit defining in order to avoid confusion (Seligman, 2012). Employee well-being is defined as a composite state that encompasses a hedonic component (life satisfaction) and a eudaimonic component (living life according to one's values) (Forgeard, Jayawickreme, Kern, \& Seligman, 2011) within an occupational context (van der Doef and Maes 1999). Past research argues that high workloads erode nurses' well-being because wellbeing is the barometer indicating how well they are coping (Brunetto et al., 2017). Previous research shows that high wellbeing predicts low turnover for public sector Australian nurses (Brunetto, Rodwell, Shacklock, Farr-Wharton, \& Demir, 2016a) and high work harassment is associated with low wellbeing for Australian and Italian public and private sector nurses (Brunetto et al., 2016b).

\section{Turnover Intentions}

Turnover intentions refers to employees' propensity to withdraw from their present workplace and consequently, they conserve their energy at work and redirect it towards looking at alternatives (Van Knippenberg, Van Dick, \& And Tavares, 2007). There are multiple push factors likely to promote turnover intentions, including poor quality relationships and the lack of support given to staff (Van Knippenberg et al, 2007; Lashinger \& Fida, 2014) as well as low wellbeing for US and Australian nurses (Brunetto et al., 2013). In each case, high wellbeing 
was associated with low turnover intentions. The following hypotheses replicate this finding for Bangladeshi nurses.

\section{H3. Employee wellbeing is negatively related to turnover intention}

\section{Mediation and Moderations}

The way that work harassment and wellbeing impact the relationship between LMX and turnover for Bangladeshi public and private sectors is not clear. There are two types of possible effects. First, work harassment moderates the association between LMX and wellbeing, and between LMX and turnover intentions. If this is the case then it means that work harassment affects the direction or strength of the relationship between LMX and wellbeing, and LMX and turnover intentions (Baron \& Kenny, 1986). Second, wellbeing mediates the association between LMX and turnover intentions, which means that employee wellbeing accounts for some of the negative relationship between LMX and turnover intentions. The two types of effects will be discussed further in the following paragraphs.

In terms of COR theory, wellbeing is the measure of an individual's personal resources and is affected by both personal (such as psychological capital) and organisational (such as LMX) factors. In particular, high LMX is associated with high wellbeing for Australian public sector nurses (Brunetto et al., 2016). In addition, high wellbeing is associated with low turnover intentions for Australian and US nurses (Brunetto et al., 2013). This means that if employees have high wellbeing (perhaps because of high personal resources), it may mediate the relationship between LMX and turnover intentions. According to COR theory, if wellbeing is high enough, we expect that the personal resources gained from high wellbeing will mediate the impact of LMX on turnover. The following hypothesis expresses this premise.

\section{H4. Employee wellbeing mediates the relationship between LMX and turnover intention}

Moderation refers to a situation where the relationship between two variables depends on a third variable. As stated, previous research shows that high LMX is associated with high 
wellbeing and high work harassment is associated with low wellbeing. In the context of Bangladeshi nurses, we propose that high work harassment results in employees feeling overworked, under-resourced, and that overall their employing organisation's expectations are unrealistic. In such a work environment, even if employees have a good relationship with their manager, it is expected that high work harassment will reduce the positive impact of LMX and employee wellbeing, and decrease the negative impact of LMX on turnover intention. Moreover, we expect that work harassment will moderate the impact of LMX because a manager is considered an agent of the organisation (Eisenhardt, 1989), and thus unrealistic expectations may flow through the manager to the nurses. Therefore, if a nurse thinks expectations are unrealistic, they may associate such expectations with the manager, which when work harassment is high will reduce the positive influence of the manager on employee wellbeing and reduce the negative affect on turnover intentions.

\section{H5. Work harassment moderates the relationship between LMX and wellbeing}

\section{H6. Work harassment moderates the relations between LMX and turnover intentions}

\section{The public-private context}

The context of this research is a comparison of the public and private sector work environment for Bangladeshi nurses. The public sector in Bangladesh is highly bureaucratic and hierarchical, and is described as being over-governed (micro-managed and autocratic) with insufficient or inappropriate governance (subject to corruption) (Ahmed \& Shirahada, 2017). This means that there is a lack of separation between the official practices of public sector officials and personal interests, and a growing realisation that recent reforms, whilst in theory about re-distributing resources from the rich to the poor, did not achieve the stated goals (Khan et al, 2014). Also despite specific legislation promoting equal rights for women, reforms have not made much of an indentation on the glass ceiling imposed upon women by the dominant 
culture in play (Jamil, Aminuzzaman \& Hoque, 2013). According to Ahmad et al. (2007) (cited in Ghuman \& Singh, 2013) reforms were supposed to provide greater access to healthcare for the poor. However, in practice, the rich were given greater access to resources, and nurses were dissuaded against going to poorer regions because of inadequate infrastructure facilities. In summary, reforms did not achieve a greater focus on efficiency or achieving outcomes. Hence, LMX, work harassment, wellbeing and turnover is expected to be worse for public sector nurses. The hypothesis is:

H7: Private sector employees perceive higher LMX and wellbeing and lower work harassment and turnover compared with public sector nurses.

\section{Method}

\section{Sample and procedure}

In the first instance, nurses who had completed an undergraduate and/or postgraduate nursing qualification from the Grameen Caledonian College of Nursing, Dacca, Bangladesh were invited to complete the survey. In total, 191 useable surveys were returned (redemption rate of $62 \%$ ). Additionally, 126 useable surveys were received from nurses working in two public sector hospitals in Dacca, Bangladesh. The data was collected in 2017 and 2018.

In total, 317 useable surveys were obtained, 131 from the public sector and 186 from the private sector. The demographic data depicts that unlike the ageing nursing workforce in many OECD countries (World Health Organization, 2018); the majority of nurses sampled in the Bangladesh public and private sectors were less than 31 years of age. As well, many have less than one-year employment with their current hospital, with the majority having less than 5 years employment in their current hospital. In the public sector, there were 34.4 per cent males $(n=45)$ and 65.6 per cent females $(n=86)$, which is a stark comparison to the private sector with 4.3 per cent males $(n=8)$ and 95.6 per cent females $(n=178)$. 


\section{Measures}

All items for each scale were scored on a six-point Likert scale from 1 (strongly disagree) to 6 (strongly agree). The reliability for each scale is depicted in Table 1.

Leader-member exchange. To measure a nurse's relationship with their line manager, we used the Leader-Member Exchange (LMX) instrument developed by Graen and Uhl-Bien (1995). An example of an item is 'My manager is satisfied with my work.'

Employee wellbeing. We measured an employee's sense of their wellbeing with the four items from Brunetto, Farr-Wharton, and Shacklock (2011). A sample item includes 'Overall, I am reasonably happy with my work life.’

Turnover intentions. To identify nurse intentions to leave their employing organisation, we used three-items adopted from Meyer, Allen, and Smith (1993) scale of turnover intentions, including, 'I frequently think about leaving my current employer.'

Work harassment. Rayner and Cooper (2006) developed a scale to measure task-attack, personal-attack and intimidation. To measure work harassment, we used three items that examine 'task-directed attack,' including the item '[This hospital] sets unrealistic targets.'

Control variables. Many variables within the nursing and turnover intention literature may impact turnover intentions. We controlled for tenure within a hospital because research has found those with longer tenure have a lower intention to leave their employing organisation (Ng \& Feldman, 2009b). Although there is limited agreement in the literature, we also controlled for gender, with one study suggesting women quit their job less than their male counterparts (e.g., Lyness \& Judiesch, 2001), and a larger scale study of 475,000 professionals and managers concluding women were more likely to voluntarily leave. Finally, we controlled for age because of $\mathrm{Ng}$ and Feldman's (2009a) postulation that older employees typically develop stronger ties to their colleagues and their employing organisations. 


\section{Data analysis}

To test a moderated mediation model, we used SPSS v.25 and the PROCESS Macro by Andrew Hayes (Hayes, 2018). The PROCESS macro is a better alternative to test mediation when compared to approaches such as that prescribed by Baron and Kenny (1986), as there is no requirement for the data to be normally distributed. Within the PROCESS macro, we used model 8 and applied 95 per cent bootstrap confidence intervals across a sample of 5000. To confirm the factor structure and to account for common method variance, we undertook a confirmatory factor analysis of the public and private sectors. To check and control for common method variance, we included a common latent factor (Podsakoff, Mackenzie, Lee, \& Podsakoff, 2003).

\section{Results}

Descriptive statistics and intercorrelations

Table 1 depicts the descriptive statistics, scale reliability and intercorrelations. Notably, LMX did not significantly influence turnover intentions in the public sector. Although not significant, the $p$-value was nearing statistical significance $(p=0.06)$. Confirmatory factor analysis

The hypothesised measurement model resulted in a poor fitting model for the private $(\mathrm{CMIN} / \mathrm{DF}=2.330, \mathrm{RMSEA}=0.085, \mathrm{CFI}=0.886$ and $\mathrm{SRMR}=0.066)$ and public $(\mathrm{CMIN} / \mathrm{DF}$ $=3.222, \mathrm{RMSEA}=0.131, \mathrm{CFI}=0.857$ and $\mathrm{SRMR}=0.081)$ sectors. Due to factor loadings below 0.5, a number of observed items were removed. We removed three LMX questions: 1. 'Regardless of how much formal authority he/she has built into his/her position, what are the chances that your leader would use his/her power to help you solve problems in your work? (What are the changes that you would)'; 2. 'have enough confidence in my leader that I would defend and justify his/her decision if he/she were not present to do so?'; and 3. 'How would you characterize your working relationship with your leader?'. We also removed 
one wellbeing item, 'I get enough time to reflect on what I do in the workplace'. The modified measurement model fit the data well for the private $(\mathrm{CMIN} / \mathrm{DF}=1.89, \mathrm{RMSEA}=.069, \mathrm{CFI}=$ .945 and $\mathrm{SRMR}=.058)$ and public $(\mathrm{CMIN} / \mathrm{DF}=1.98, \mathrm{RMSEA}=.087, \mathrm{CFI}=.953$ and $\mathrm{SRMR}$ $=.067)$ sectors. We tested a number of alternate models to establish the distinctiveness of the variables and the modified measurement model. As depicted in Table 2, the fit of the alternate models was significantly worse when compared with the modified measurement model for the private and public sectors.

The addition of a common latent factor (CLF) to the modified measurement model (model 4) for the private and public sectors resulted in improved model fit. In addition, a chisquare difference test for each sample revealed that the CLF model was statically different from the modified measurement model, and thus common method variance may explain a significant portion of the variance. To identify where common method variance was problematic, we compared the standardised factor loadings of the modified measurement model and the CLF model. To the best knowledge of the authors, there are no published cut-off values for the comparison of standardised factor loadings. We considered any loading difference above 0.20 to be excessively large and retained the CLF control. The final measurement model for the private and public sector include the bias-corrected estimates (i.e., controlling for common method bias). Specifically, to be able to test the hypotheses within the PROCESS macro, we imputed the bias-correct composite variables from AMOS into SPSS.

\section{Testing the hypotheses}

Figure 1 depicts the results for hypotheses 1-3 for the public and private sector. LMX predicts turnover $(\mathrm{H} 1)$ for private sector employees only and LMX predicts wellbeing $(\mathrm{H} 2)$ for both public and private sector and wellbeing predicts turnover intentions (H3) for public sector employees only. There was support for the acceptance of hypothesis 4 (mediation), with a significant indirect effect $\mathrm{SE}=-.21$, CI (-.42,-.10). As mentioned, the direct effect between LMX 
and turnover intentions was not significant, providing support that wellbeing completely mediates the relationship between LMX and nurse turnover intentions in the Bangladesh public sector. We also found work harassment did not moderate the relationship between LMX and turnover intentions (i.e., rejecting hypothesis 6), but, as depicted in Figure 2, work harassment did moderate the path between LMX and employee wellbeing (i.e., providing support for the acceptance of hypothesis 5). Specifically, high work harassment reduced the influence of LMX on employee wellbeing.

In relation to the private sector, wellbeing did not directly relate to turnover intentions, so we rejected hypothesis 2 . Due to the rejection of hypothesis 3 , and insignificant indirect effects, we rejected hypothesis 4 . Finally, work harassment did not moderate the relationship between LMX and wellbeing, or LMX and turnover intentions. As such, we rejected hypotheses 5 and 6.

\section{Discussion}

This paper examines the impact of line management on work harassment, wellbeing and turnover of public and private nurses in Bangladesh using a COR framework. One factor which has been identified as having a negative impact, especially in the public sector, is work harassment and in developed countries, the manager either alleviates the situation or makes it worse (Brunetto et al, 2016b). However, research in Brazil found that management did not have the same impact compared with Anglo-American and/or European countries such as UK and Australia (Brunetto et al, 2015), hence, we examined the impact of management on public and private nurses' perceptions of work harassment in Bangladesh. Additionally, since, private sector nurses operate in hospitals that have attempted to implement some typically western HR systems, it seems likely that turnover should be less because the influence of culture (corruption etc) should be less. 
In terms of the first and second hypotheses, previous research had already shown that effective management is associated with high wellbeing and low turnover intentions for public and private sector employees in Anglo-American and European countries (Martin et al., 2016; Brunetto et al., 2018, 2013). The findings from this research support previous research showing that high LMX is associated with high wellbeing for both public and private sector nurses in Bangladesh. The role of the line manager is to act as a source of support when workloads are physically and mentally draining - hence in the ideal situation, they provide a barrier to stress, in turn supporting high wellbeing (Lashinger \& Fida, 2014). However, high LMX is only associated with low turnover for private sector nurses in Bangladesh. One explanation is that public sector nurses are unlikely to have a modern HR system in place (of which a basic manager-employee relationship is an important cornerstone), and instead, the traditional culture may be more important in determining whether employees stay or leave (Ahmed \& Shirahada, 2017; Khan et al., 2014). Khan et al (2014) argues that in Bangladesh, the policy may be similar to a developed country, but the implementation of HR policies has been challenging, especially in the public sector. Hence, it seems likely that family ties and corruption still dominate decision making, in turn negating the role of managers.

Additions, the findings suggest that in contrast to the role of line managers of professionals in Anglo-American and European countries, who play two distinct (sometimescontradictory) roles - professional hybridity, firstly developing nurses' professional skills and knowledge, while still acting as their line manager (Noordegraaf, 2015); the role of public sector line managers in Bangladesh is unclear because nurses' wellbeing is determined by how work harassment mediates the relationship between LMX and wellbeing. One possible reason is that their managers are not engaging in professional activities such as socialising them into the professional norms and values associated with nursing. Khan et al (2014) suggests that in countries like Bangladesh, increased professionalization of the Bangladeshi public sector has 
failed to change 'who gets what' and how workplaces are organised (Jamil, 2013). Hence LMX may not be based on professional factors and the managers may not act as positive antecedents of nurses' wellbeing as is the case in developed countries (Martin et al, 2016). However, further research should examine why a significant relationship was absent.

Regarding hypothesis 3, previous research had already shown that high wellbeing was associated with low turnover intentions for nurses in the USA and Australia (Brunetto et al., 2016). In terms of COR theory, high wellbeing is an important personal resource that employees want to develop, and conserve when required (Hobfoll 2011). The findings from this research are consistent with past research and COR theory for public sector Bangladeshi nurses only. More research is required to investigate the relationship for private sector nurses. It may be that the traditional culture has a role to play in better understanding the relationship between wellbeing and turnover for Bangladeshi nurses working in the private sector (Khan et al., 2014). Such insight may better explain how employees build wellbeing in the workplace, and importantly, why they stay even in workplace enrivonments that deplete their wellbeing.

The mediation and moderation processes shed further light on understanding the differences in the public and private workplaces for nurses in Bangladesh. Past research shows that work harassment is a product of the era of austerity, which now governs the work lives of many professionals in the caring industry, especially in the public sector in developed countries. However, very little is known about how reforms (if any?) have affected the professional workplace in developing countries. In ideal circumstances, professionals expect their line managers to mediate unrealistic organisational work demands and provide appropriate and sometimes-extra support resources to ensure both task completion and professional development occurs (Farr-Wharton et al., 2011). In this study, work harassment does mediate the relationship between LMX and wellbeing for public sector nurses, but not for private sector 
nurses. Over half of public sector Bangladeshi nurses' wellbeing is explained by how work harassment mediates the relationship between LMX and wellbeing.

While previous research shows that LMX predicts work harassment (Farr-Wharton et al., 2018), and work harassment predicts employees' wellbeing (Xerri et al., 2016), this paper adds new evidence about the role of work harassment in moderating the relationship between management and wellbeing of public sector nurses in Bangladesh. More specifically, in the public sector, when work harassment was low, management had a greater positive influence on employee wellbeing. These results provide new evidence about the influence of work harassment on the well-established association between management practices and wellbeing. This means that the role of the manager is just as important in developing countries such as Bangladesh as it is in developed countries, although culture may affect how it works. It also aligns with COR theory in that those with fewer resources are less able to apply resources to develop other resources, which suggests that those managers who support their nurses impact their decision to stay by improving their wellbeing. However, this is the case for public sector nurses only.

In contrast, LMX explains just under a quarter of private sector nurse wellbeing, and work harassment has no significant impact on this association. Further, there is no evidence of work harassment moderating the relationship between LMX and turnover in this study, although, high LMX, work harassment and wellbeing does explain just under a third of the turnover intentions of public sector Bangladeshi nurses. In the case of private sector Bangladeshi nurses, work harassment alone explains well over a third of their turnover intentions. This suggests that the manager may not have the power to affect how work harassment influences turnover intentions for private sector nurses. This is in contrast to research findings in the Anglo-American and/or European countries which may suggest a significantly different work context for these private sector nurses working in one of the least 
developed countries in the world. The findings provide a platform for more research about management issues facing public and private professionals such as nurses in developing countries such as Bangladesh.

The last hypothesis examined the differences between the public and private sector nurses' level of satisfaction with LMX, work harassment, wellbeing and turnover. The belief was that private sector nurses would perceive higher LMX and wellbeing and lower work harassment and turnover intentions compared to their private sector counterparts. The hypothesis was based on information that public sector reforms have not been successfully implemented, the public sector in Bangladesh is poorly resourced, and nurses are actively dissuaded from going to poorer regions because of inadequate infrastructure facilities (Jamil, 2013). However, the hypothesis was rejected because, across every factor, public sector nurses were more satisfied with management, had higher wellbeing, perceived lower work harassment and turnover intentions (See Table 1). One explanation using COR theory is that private sector nurses' wellbeing is being conserved and developed from other resources not examined in this paper. Future studies should further examine the antecedents, mediators and moderators relating to work harassment.

In reviewing this study, there are some limitations to consider. First, the cross-sectional data was susceptible to common method bias (Podsakoff et al., 2003). However, as mentioned, a common latent factor was included, and several items that were explained by common method variance were controlled. Nonetheless, future research should examine work harassment over time to improve insight and design relating to common method bias. Second, although a study on Bangladeshi nurses provides some insight into work harassment in developing countries, further research is required about other developing countries. Further, another limitation relates to the sample in that most of the nurses had worked less than five years in their current employment, and therefore, there may be other factors, other than LMX 
and wellbeing affecting their intention to stay that have not been examined in this paper. Finally, the findings are limited by the sample size for each sector, and the type of demographic information collected that could have assisted in explaining it. Further research should be undertaken to increase the sample size, which will improve the stability of the results, and provide enough statistical power to examine more complex research models, including a thourough examination of work harassment antecedents, mediators and moderators.

\section{Conclusion}

The findings suggest that work harassment is prevalent in the Bangladesh public and private nursing profession. This study used a COR theoretical framework to compare the impact of management practices (LMX) and work harassment on the wellbeing and turnover intentions of Bangladeshi public and private nurses. Using COR theory, we found that LMX is a positive resource caravan passageway that can build wellbeing for public sector employees; however, the association was not significant for private sector nurses. Hence, COR theory principle 2 offers one explanation of $\mathrm{r}$ public sector employees' behaviour in that it assumes thate the manager provided enough of a 'cushion' for them to form a barrier against the negative influence of work harassment on their wellbeing (Hobfoll, 2001). Similar to per past AngloAmerican and European research has shown, the consequences of poor environmental conditions, such as work harassment, is that it does affect how public sector nurses in Bangladesh are able to take advantage of the relationship with their manager. However, there was no evidence that employees who perceive high support from their line managers are less likely to be negatively influenced by work harassment (Hobfoll, 2011)..Therefore, whilst COR theory was somewhat useful in explain some behaviour of public sector Bangladeshi nurses, it was far less relevant for explaining the behaviour of those in the private sector. This is an important finding because theories and concepts developed and validated in Anglo-American 
contexts assume underlying cultural similarities that may not be accurate in developing countries. Future research should perhaps test other well established theories (and concepts) across multiple contexts to verify their validity.

The implication of this research relates directly to public sector management. For example, the impact of management is different in Bangladesh compared with past AngloAmerican and European studies, and one factor is likely to be the traditional cultural stronghold, especially the impact of ties and corruption, which affects nurses' turnover intention, especially in public sector nurses areas (Ahmed \& Shirahada, 2017). Future research should explore those assertions. Additionally, there are significant differences in how public and private sector nurses respond to, and cope with work harassment. Hence, simply transplanting management models from developed countries for managing healthcare in developing countries may not yield its intended benefits. More research is required to better understand how managers affect how professionals work in developing countries. 


\section{References}

Ahmed, T \& Shirahada, K (2017) Toward a sustainable healthcare service system in a limited resource context: Case study of Bangladesh rural advancement committee's healthcare system, International Journal of Healthcare Management,

DOI: $10.1080 / 20479700.2017 .1389512$

Baron, R. M., \& Kenny, D. A. (1986). The moderator-mediator variable distinction in social psychological research: Conceptual, strategic, and statistical considerations. Journal of Personality and Social Psychology, 51(6), 1173-1182.

Branch, S., Ramsay, S., \& Barker, M. (2013). Workplace bullying, mobbing and general harassment: A review. International Journal of Management Reviews, 15, 280-299

Brunetto, Y., Farr-Wharton, R., \& Shacklock, K. (2011). Using the Harvard HRM model to conceptualise the impact of changes to supervision upon HRM outcomes for different types of public sector employees International Journal of Human Resource Management 22(3), 553-573.

Brunetto, Y., Rodwell, J., Shacklock, K., Farr-Wharton, R., \& Demir, D. (2016a). The impact of individual and organizational resources on nurse outcomes and intent to quit. Journal of advanced nursing, 72(12), 3093-3103.

Brunetto, Y., Xerri, M., Shriberg, A., Farr-Wharton, R., Shacklock, K., Newman, S., \& Dienger, J. (2013). The impact of workplace relationships upon engagement, wellbeing, commitment and turnover for nurses in Australia and USA. Journal of advanced nursing, 69(12), 27862799.

Brunetto, Y., Xerri, M., Trinchero, E., Beattie, R., Shacklock, K., Farr-Wharton, R., \& Borgonovi, E. (2018). Comparing the impact of management on public and private sector nurses in the UK, Italy, and Australia. Public Management Review, 20(4), 525-544.

Brunetto, Y., Xerri, M., Trinchero, E., Farr-Wharton, R., Shacklock, K., \& Borgonovi, E. (2016b). Public-Private Sector Comparisons Of Nurses' Work Harassment Using Set: Italy and Australia. Public Management Review, 18(10), 1479-1503. doi:DOI:10.1080/14719037.2015.1114136

Brunetto, Y., Shacklock, K., Teo, S., Farr-Wharton, R., \& Nelson, S. (2015). Nurses' managers, learning options and organisational commitment: Australia, Brazil and England. Journal of Nursing Management. $23(8), 1029-1038$

Dick, G. P. (2010). Can the organisation and supervision environment influence both bullying and organisational commitment? Evidence from a police force survey. Kent University Working Paper Series 196.

Diefenbach, T. (2009). New public management in public sector organizations: the dark sides of managerialistic 'enlightenment'. Public Administration, 87(4), 892-909.

Einarsen, S. (2000). Harassment and bullying at work: A review of the scandinavian approach. Aggression and Violent Behavior, 5(4): 379-401

Einarsen, S., Hoel, H., \& D. Zapf, D. eds. (2011). Bullying and Harassment in the Workplace: Developments in Theory, Research and Practice ( $2^{\text {nd }}$ Edition). CRC Press, Taylor \& Francis Group, Boca Raton, Florida.

Einarsen, S., \& Nielsen, M. (2015). Workplace bullying as an antecedent of mental health problems: A five-year prospective and representative study. International Archives of Occupational and Environmental Health. 88: 131-142

Eisenhardt, K. M. (1989). Agency theory: An assessment and review. Academy of Management Review, 14(1), 57-74.

Farr-Wharton, R., Brunetto, Y., \& Shacklock, K. (2011). Professionals' manager-subordinate relationships, autonomy and commitment in Australia: a leader-member exchange theory perspective. The International Journal of Human Resource Management, 22(17), 3496-3512. 
Forgeard, M. J., Jayawickreme, E., Kern, M. L., \& Seligman, M. E. (2011). Doing the right thing: Measuring wellbeing for public policy. International journal of wellbeing, 1(1), 79-106.

Ghuman, B. S., \& Singh, R. (2013). Decentralization and delivery of public services in Asia. Policy and Society, 32, 7-21

Graen, G., \& Uhl-Bien, M. (1995). Relationship-based approach to leadership: Development of leader-member exchange (LMX) theory of theory over 25 years: Applying a multi-level multidomain perspective. Leadership Quarterly, 6(2), 219-247.

Halbesleben, J. R., Neveu, J.-P., Paustian-Underdahl, S. C., \& Westman, M. (2014). Getting to the "COR" understanding the role of resources in conservation of resources theory. Journal of management, 40(5), 1334-1364.

Hayes, A. F. (2018). Introduction to mediation, moderation, and conditional process analysis: A regression-based approach. New York: Guilford Press.

Hobfoll, S. E. (1989). Conservation of resources: A new attempt at conceptualizing stress. American psychologist, 44(3), 513-524.

Hobfoll, S. E. (2001). The influence of culture, community, and the nested-self in the stress process: advancing conservation of resources theory. Applied psychology, 50(3), 337-421.

Hobfoll, S. E. (2011). Conservation of resource caravans and engaged settings. Journal of occupational and organizational psychology, 84(1), 116-122.

Jamil, I., Aminuzzaman, S., \& Haque, S. (2013) Introduction: Governance in South, Southeast, and East Asia Public Organization Review 13 (4) 341- 347

Khan., R, Miah., M, \& Manzoor., A (2014). Human Resource Management Practices: A Case Study of South Asian Countries IBT Journal of Business Studies 9(1), 83-101

Lyness, K. S., \& Judiesch, M. K. (2001). Are female managers quitters? The relationships of gender, promotions, and family leaves of absence to voluntary turnover. Journal of applied Psychology, 86(6), 1167-1178.

Mahdy, H. A. (2009) Reforming the Bangladesh healthcare system, International Journal of Health Care Quality Assurance, 22(4), 411-416.

Martin, R., Thomas, G., Guillaume, Y., Lee, A. \& Epitropaki, O. (2016). Leader-member Exchange

(LMX) and Performance: A Meta-analytic Review. Personnel Psychology, 69, 67-121.

Meyer, J. P., Allen, N., \& Smith, C. A. (1993). Commitment to organizations and occupations: extension and test of a three-component conceptualization. Journal of applied Psychology, 78(4), 538-551.

$\mathrm{Ng}, \mathrm{T}$. W., \& Feldman, D. C. (2009a). Age, work experience, and the psychological contract. Journal of Organizational Behavior, 30(8), 1053-1075.

$\mathrm{Ng}, \mathrm{T}$. W., \& Feldman, D. C. (2009b). Re-examining the relationship between age and voluntary turnover. Journal of Vocational Behavior, 74(3), 283-294.

Noordegraaf, M. (2015) Hybrid Professionalism and Beyond: (New) Forms of Public Professionalism in Changing Organizational and Societal Contexts, Journal of Professions and Organization, 2, 187-206.

Podsakoff, P. M., MacKenzie, S. B., Lee, J.-Y., \& Podsakoff, N. P. (2003). Common method biases in behavioral research: a critical review of the literature and recommended remedies. Journal of applied Psychology, 88(5), 879-903.

Rayner, C., \& Cooper, C. L. (2006). Workplace bullying. In K. E. Kelloway, J. Barling, \& J. J. H. Jr. (Eds.), Handbook of workplace violence. Thousand Oaks: Sage Publications.

Laschinger, H.K.S., Fida, R., (2014). New nurses' burnout and workplace wellbeing: the influence of authentic leadership and psychological capital. Burnout Research 1 (1), 19-28

Seligman, M.E., (2012). Flourish: A visionary new understanding of happiness and well-being. New York: Simon and Schuster.

Tummers, L., Vermeeren, B., Steijn, B., \& Bekkers, V. (2012). Public professionals and policy implementation: Conceptualizing and measuring three types of role conflicts. Public Management Review, 14(8), 1041-1059. 
Tummers, L., Brunetto, Y., Teo, S., (2016) Workplace aggression: Introduction to the special issue and future research directions for scholars, International Journal of Public Sector Management, 29 (1), $2-10$

Van Knippenberg, D., Van Dick, R., \& and Tavares, S. (2007). Social Identity and Social Exchange: Identification, Support, and Withdrawal from the Job. Journal of Applied Social Psychology, 37(3), 457-477.

Van der Doef, M. and Maes, S., 1999. The job demand-control (-support) model and psychological well-being: a review of 20 years of empirical research. Work \& stress, 13(2), pp.87-114.

World Bank. (2016). Overview of Bangladesh. Retrieved from http://www.worldbank.org/en/country/bangladesh/overview

World Health Organization. (2016). World Health Statistics 2016: Monitoring Health for the Sustainable Development Goals (SDGs). World Health Organization. Retrieved from http://www.who.int/gho/publications/world health statistics/2016/en/

World Health Organization. (2018). Nursing and Midwifery Fact Sheet February 2018. Retrieved from http://www.who.int/mediacentre/factsheets/nursing-midwifery/en/

Xerri, M., Farr-Wharton, R., Brunetto, Y., \& Lambries, D. (2016). Work harassment and local government employees: Australia and USA. International Journal of Public Sector Management, 29(1), 54-71.

Zapf, D., Escartin,J., Einarsen, S., Hoel, H. \& Vartia, M. (2011). Empirical findings on the prevalence and risk groups of bullying in the workplace. In Bullying in the Workplace; Developments in Theory, Research and Practice edited by Einarsen, S., Hoel, H., Zapf, D., Cooper. C. CRC press, Taylor \& Francis group, Boca Rata 
Table 1. Correlation matrix

\begin{tabular}{|c|c|c|c|c|c|c|c|c|c|c|c|}
\hline & $\begin{array}{l}\text { Private } \\
\mathrm{M}(\mathrm{SD})\end{array}$ & $\begin{array}{l}\text { Public } \quad \text { C. } \\
\mathrm{M}(\mathrm{SD})\end{array}$ & $\mathrm{R}$ AVE & E $\sqrt{A V E}$ & 1 & 2 & 3 & 4 & 5 & 6 & 7 \\
\hline 1. Gender & - & - & & & 1 & .09 & $.22 *$ & -.10 & $.22 *$ & -.06 & $.32 *$ \\
\hline 2. Age group & - & - & & & -.07 & 1 & $.20^{*}$ & $.28 * *$ & -.13 & $.18^{*}$ & -.06 \\
\hline 3. Hospital tenure & - & - & & & .04 & $.36^{* *}$ & 1 & -.08 & -.07 & .03 & .04 \\
\hline $\begin{array}{l}\text { 4. Turnover } \\
\text { intentions }\end{array}$ & $2.86(1.32)$ & $1.64(.84) .87$ & .70 & .84 & .09 & $-.26 * *$ & $-.19 *$ & & $-.29 * *$ & $.50 * *$ & -.15 \\
\hline 5. Wellbeing & $3.90(.50)$ & $4.41(.94) \quad .93$ & .81 & .90 & .05 & -.00 & -.05 & -.08 & 1 & -.14 & $.68 *$ \\
\hline $\begin{array}{l}\text { 6. Work } \\
\text { harassment }\end{array}$ & $2.74(1.30)$ & $2.61(1.17) .87$ & .69 & .83 & .07 & -.13 & -.07 & $.81 * *$ & -.13 & 1 & $-.21^{*}$ \\
\hline $\begin{array}{l}\text { 7. Leader-member } \\
\text { exchange }\end{array}$ & $3.30(.65)$ & $4.63(1.08) .88$ & .64 & .80 & -.00 & .07 & -.05 & $-.38 * *$ & $.52 * *$ & $-.37 * *$ & 1 \\
\hline $\begin{array}{l}* \text { Correlation is sig } \\
0.01 \text { level ( } 2 \text {-tailed). } \\
\text { average variance ext } \\
\text { scale where } 1=\text { Stron }\end{array}$ & $\begin{array}{l}\text { nificant at } t \\
\text { Private sec } \\
\text { tracted; } \sqrt{A} \\
\text { gly Disagre }\end{array}$ & $\begin{array}{l}\text { the } 0.05 \text { lev } \\
\text { ctor below t } \\
\text { VE = squar } \\
\text { ee, } 2=\text { Disag }\end{array}$ & $\begin{array}{l}-\mathrm{ta} \\
\mathrm{iag} \\
\mathrm{t}\end{array}$ & $\begin{array}{l}* * \\
\text { C.R } \\
\text { AV }\end{array}$ & 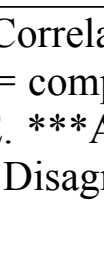 & $\begin{array}{l}\text { ion } \\
\text { osit }\end{array}$ & $\begin{array}{l}\text { ignif } \\
\text { liabil }\end{array}$ & $\begin{array}{l}\text { fican } \\
\text { ility; } \\
\text { e bas }\end{array}$ & $\begin{array}{l}\text { the } \\
\mathrm{E}= \\
\text { n a } \\
\text { ree, }\end{array}$ & & \\
\hline
\end{tabular}

Table 2. Comparison of alternate measurement models

\begin{tabular}{|c|c|c|c|c|c|c|c|}
\hline Model & & $\chi^{2}$ & $\Delta \chi^{2}$ & df & $\Delta \mathbf{d f}(p)$ & CFI & SRMR \\
\hline & $\begin{array}{l}\text { Hypothesised measurement model } \\
\text { Private sector }\end{array}$ & 265.217 & & 113 & & .886 & .066 \\
\hline Base & $\begin{array}{l}\text { Modified measurement model - private } \\
\text { sector }\end{array}$ & 111.608 & & 59 & & .945 & .058 \\
\hline 1 & Combine LMX and wellbeing & 181.715 & 70.11 & 62 & $3(\mathrm{p}<.001)$ & .874 & .084 \\
\hline 2 & Combine LMX and Harassment & 317.966 & 206.36 & 62 & $3(\mathrm{p}<.001)$ & .731 & .146 \\
\hline 3 & Combine wellbeing and harassment & 229.781 & 118.7 & 62 & $3(\mathrm{p}<.001)$ & .824 & .118 \\
\hline \multirow[t]{2}{*}{4} & Add CLF to Base model & 76.394 & 35.21 & 46 & $13(\mathrm{p}<.001)$ & .968 & .045 \\
\hline & $\begin{array}{l}\text { Hypothesised measurement model (four } \\
\text { factor model) - Public sector }\end{array}$ & 364.098 & & 113 & & .857 & .081 \\
\hline Base & $\begin{array}{l}\text { Modified measurement model - public } \\
\text { sector }\end{array}$ & 116.799 & & 59 & & .953 & .067 \\
\hline 1 & Combine LMX and wellbeing & 309.179 & 192.38 & 62 & 3( & .797 & .090 \\
\hline 2 & Combine LMX and Harassment & 326.026 & 209.23 & 62 & $3(\mathrm{p}<.001)$ & .783 & .161 \\
\hline 3 & Combine wellbeing and harassment & 332.380 & 215.58 & 62 & $3(\mathrm{p}<.001)$ & .778 & .164 \\
\hline 4 & Add CLF to Base model & 69.596 & 42.203 & 46 & $13(\mathrm{p}<.001)$ & .981 & .046 \\
\hline
\end{tabular}




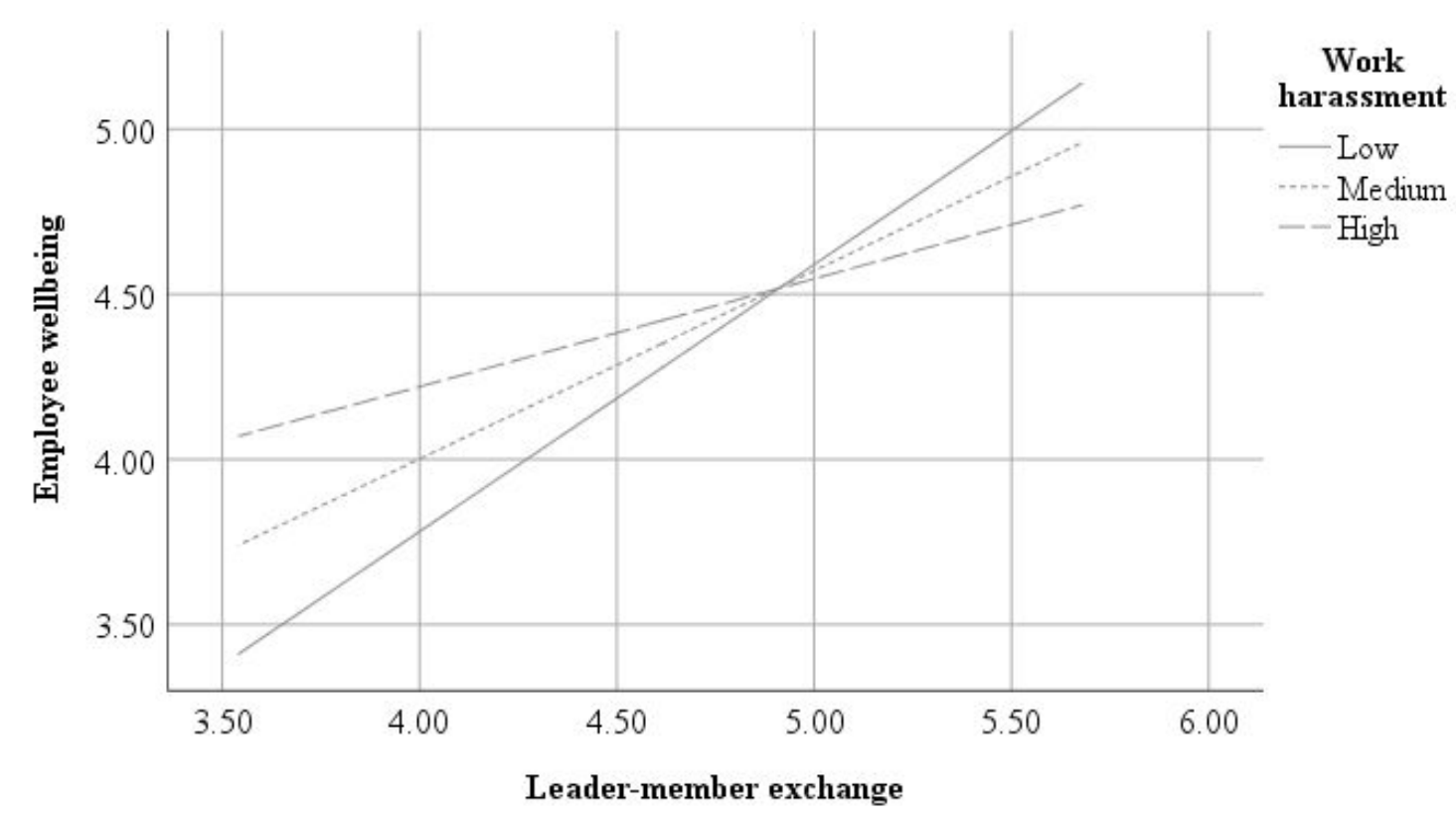

Figure 2. Moderation results - work harassment moderates the relationship between LMX and employee wellbeing. Note: Work harassment standardised for moderation analysis 


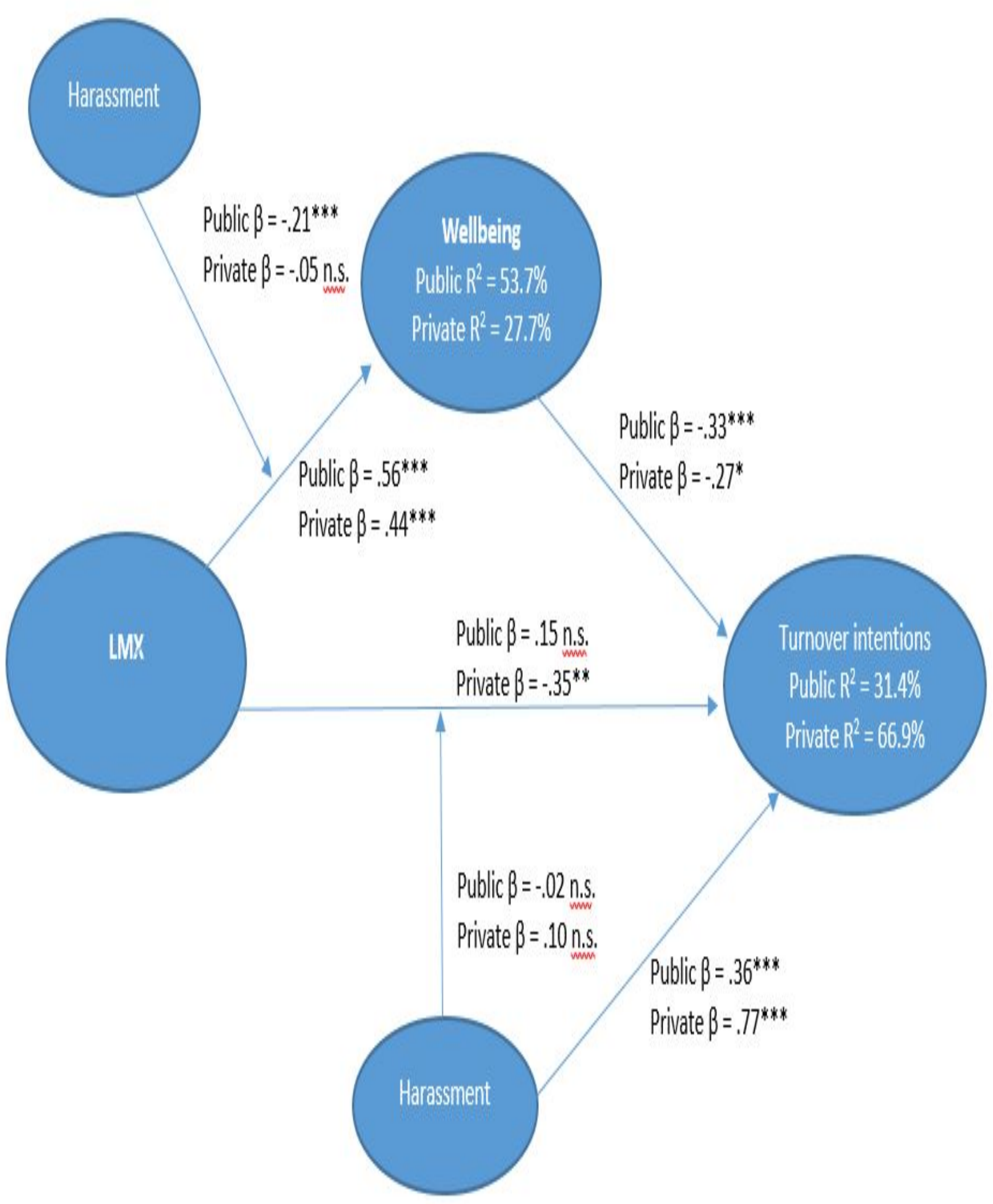




\title{
Comparing the impact of management on public and private nurses in Bangladesh
}

\begin{abstract}
Purpose: This paper uses Conservation of Resources (COR) theory as a lens for comparing the impact of line management on Bangladeshi public and private nurses' perception of work harassment, wellbeing and turnover intentions where Anglo-American and European management models have been super-imposed on an existing different culture.

Design: Survey data was collected from 317 Bangladeshi nurses' (131 from the public sector and 186 from the private sector). Structural Equation Modelling was used for analysis.

Findings: High work harassment was associated with low wellbeing and management practices, and explained approximately a quarter of private sector nurse-s's wellbeing. In total, management, work harassment and employee wellbeing explained approximately a third of the turnover intentions of public sector nurses, whereas only work harassment explained approximately a third of private sector nurses' turnover intentions. The findings suggest a differential impact of management on work harassment across the public and private sector.
\end{abstract}

Limitation; Cross-sectional data is susceptible to common method bias. A common latent factor was included, and several items that were explained by common method variance were controlled. Further, the findings are limited by the sample size from one sector and the use of only one developing country.

Implications: It is a waste of resources to transplant Anglo-American and European management models to developing countries without understanding the impact on nurses' outcomes.

Originality: Anglo-American and European management models and theories are not easily transferable to the public sector Bangladesh context probably because of the impact of ties and 
corruption. Line management is a positive resource that can build employee wellbeing for public sector employees only.

Keywords: public-private sector, line management, well-being, work harassment, turnover intentions 


\section{Comparing the impact of line management on public and private nurses in Bangladesh}

Public sector management models do not easily translate to best practice in developing countries, mainly because the conditions are too different. For example, in contrast to developed countries, there are more doctors than nurses in Bangladesh and the demand for nurses' services exceeds supply whilst continuing to grow in line with population growth: hence they work in high-pressure environments (World Health Organization, 2018; World Bank, 2016). As a consequence, this paper examines whether Bangladeshi nurses perceive work harassment similarly to other public and private nurses in developed countries (Brunetto, Xerri, Trinchero, Farr-Wharton, Shacklock, Borgonovi, 2016) and whether this affects their turnover intention. Dick (2010) refers to work harassment as a form of workplace bullying but suggests that it differs from bullying. Specifically, an employee is exposed to work harassment when their employing organisation unreasonably monitors their actions, sets excessively high workloads, and has stringent accountability standards (Brunetto et al, 2016b). As such, the organisation and its managers are the instigators of work harassment.

Work harassment has been identified as a growing problem for public sector managers in developed countries (Tummers, Brunetto \& Teo 2016). For example, Brunetto et al. (2016b) compared work harassment of nurses in Australia and Italy and found significant parallels in their perceptions. More recent research identified a growing presence and acceptance of work harassment in US and UK hospitals (Farr-Wharton, Brunetto \& Xerri, 2019). The delivery of health services has been significantly affected by the cost-constraint that mandated the depth and breadth of reforms over the past four decades which has resulted in the expectation that employees will 'do more with less' (Diefenbach, 2009). However, the situation is even more pronounced in Bangladesh, where the shortages in nurses, especially in the public sector and in particular, in rural areas, is a growing problem (Ahmed \& Shirahada, 2017). Specifically, there are high levels of resource inadequacy, with widespread shortages in basic nursing 
equipment and a lack of an effective nurse management systems (World Health Organization, 2016). Such inadequacies in the Bangladesh system are likely to create a potential haven for work harassment.

The shortage of public sector nurses in Bangladesh is increasingly dire, and in some cases, the families of patients have to act as carers (Mahdy, 2009). If work harassment is not managed effectively, then it may negatively influence the profession's ability to attract new nurses, and retain them in the job. Whilst there is evidence of work harassment across different public sector occupations, such as local government and healthcare workers across different countries - Australia, Italy, USA (Tummers et al 2016; Xerri et al, 2016), there is no evidence as to whether work harassment is an issue in developing countries. In this study, we have two core aims. The first aim is to develop insight into the impact of line management on outcomes such as work harassment, wellbeing and turnover intentions for nurses working in Bangladeshi hospitals. The second aimis to develop a deeper understanding of the similarities and differences across the public and private sectors.

In relation to work harassment, in developed countries the theory argues that the role of the line manager for professionals is very important because of the dual role of the professional mentor and line manager within the organisational hierarchy. However, line managers of professionals are also increasingly faced with the issue of 'professional hybridity' (the sometimes-contradictory expectations that managers will both develop nurses' professional skills and knowledge and ensure task completion) (Noordegraaf, 2015). Never-the-less, a good line manager is one who can mediate the excessive demands of the hierarchy to ensure appropriate resourcing and support is available in relation to task completion and professional development (Farr-Wharton, Brunetto, \& Shacklock, 2011). Although past evidence has established the association between line management practices and wellbeing, and between line management practices and turnover intentions (Brunetto et al., 2013), to the best 
knowledge of the authors, there is no evidence about how work harassment may change these established relationships. As such, we aim to add to the existing evidence by examining the role of work harassment in affecting the association between satisfaction with line management and employee wellbeing, and in turn, turnover intentions within a developing country such as Bangladesh. Such evidence adds to the literature about the impact of line management on employee wellbeing and turnover intentions of nurses within developing countries, in this case, Bangladesh.

Managing both public and private sector employees in developing countries can be challenging as international HR systems are imported into existing culture-driven decisionmaking systems. Most managers were traditionally autocratic in their practices in South Asia (Khan, Miah \& Manzoor, 2014). However, in the last three decades, the service sector has undergone significant change mainly because of global forces and government reforms that have elevated the importance of human resources slowly, especially in terms of the significance of ensuring adequate qualifications and competencies for key positions. This change has led to the introduction of modern HRM and strategic systems, however, the new systems have not replaced the previous stronghold of culture in organisational decision-making. This means that family ties and corruption still dominate decision making, favouring some and disadvantaging the majority, and whilst those with strong international connections are likely to have basic HR policies and structures evident in countries such as the USA and UK, the difference is in their ability to implement those policies into practice (Khan et al., 2014, p 99). Hence, while Bangladeshi organisations may have specific policies about work hours and duties, in practice, there is probably greater scope for work harassment in Bangladesh compared with AngloAmerican countries such as the UK, USA and Australia.

This paper compares the relationship between line management, work harassment, wellbeing and turnover intentions of public and private sector nurses using a Conservation of 
Resources (COR) theoretical framework (Hobfoll, 1989). COR theory is typical of theories originally developed and tested within an Anglo-American and/or European context (history, culture) to explain employee outcomes regarding their ability to maintain and grow their personal resources such as health and wellbeing. However, it may not have as much relevance in explaining behaviour in developing countries. COR theorists argue that when the organisational environment erodes their personal resources through work harassment, their wellbeing is negatively impacted and this motivates them to protect their well-being by regulating their work practices. COR theorists argue that if extra support (e.g., from managers) is not available, then employees may offset perceived resource losses, and potential shortages, by reducing effort at work or leaving the organisation (Hobfoll, 2011). The research questions (RQs) guiding the study are:

$R Q$ 1: What is the impact of line management on work harassment, wellbeing and turnover of public and private sector Bangladeshi nurses?

$R Q$ 2: What are similarities and differences in the relationship between line management, work harassment, wellbeing and turnover for nurses in the public and private sector?

The next part details a relevant review of the literature and identifies hypotheses that reflect the gap in the literature.

\section{Background}

\section{Conversation of Resources (COR) theory}

The COR theory is ideal for understanding employee outcomes, especially in the face of resource adversity (Hobfoll 1989). The way COR theory works is via two principles and four Corollaries (Hobfoll, 2011). Principle one: a loss of resources likely has a bigger effect than acquiring new resources. Principle two: to gain new or more resources, employees must firstly spend time or energy (resources) in those activities likely to lead to more resources. Further, if employees believe they are likely to experience a loss of resources (such as a loss 
of wellbeing) they will likely engage in activities (sick leave/ look for another job) to protect themselves against falling wellbeing. The four corollaries comprise four assumptions Firstly, the higher employees' perception of resources are, the easier it is for them to gain resources, whereas employees with fewer resources are more susceptible to resource loss, and less able to gain resources. Secondly, it is likely that an initial loss of resources will lead to further losses; whereas it is likely that a resource gain will likely lead to further resources gains. Fourthly,) employees are likely to act defensively as a strategy to prevent further loss of resources (Halbesleben, Neveu, Paustian-Underdahl, \& Westman, 2014).

Drawing from COR theory, each organisation makes decisions that produce either positive or negative environmental conditions, called 'resource caravan passageways' (Hobfoll (2011). Under ideal conditions, an employee can build resources such as increased wellbeing by investing other resources at their disposal; in this case, effective supervision is a social resource (caravan passageway) an employee can draw upon. Whereas, if the resource caravan passageway is high levels of work harassment, employees have low resources and are thus less able to orchestrate resource gain. In this case, we propose that work harassment may result in employees that are less able to develop wellbeing with the social resource derived from the relationship with their manager.

Using COR theory to explain the relationship between management, work harassment, wellbeing and turnover, we argue that effective management practices is a positive resource caravan passageway that can build wellbeing, whereas work harassment is a negative resource caravan passageway that will influence an employee's ability to develop resources such as wellbeing. The consequences of the poor environmental conditions are likely to lead to increased turnover (Hobfoll, 2001). Using COR corollary 4, the issue will be whether the support from the manager is enough of a 'cushion' to form a barrier against the negative influence of work harassment on wellbeing, and the flow on to an intention to turnover. As per 
corollary 4 , those with a lack of resources are likely to act defensively to conserve against further resource loss. That is, those who lack resources are unable to apply resources to fight against resource loss (Hobfoll, 2011), and thus to conserve further loss, they may choose to eject themselves from the situation by intending to leave their employing organisation. In contrast, those employees with adequate resources are likely to be able to invest them in resource development, which is likely to be evident by high wellbeing and low turnover intentions.

\section{Variables under examination}

\section{Work Harassment (resource caravan passageway)}

The term 'work harassment' is challenged by different groups of researchers and the same work has multiple meanings depending on the country. Originally, the term emerged from 'harassment' meaning “... all repeated behaviors [sic] which ... provoke, frighten, intimidate, or bring discomfort to the victim" (Brodsky (1976) cited in Einarsen, 2000; p382). However, the definition has moved on in different ways in different countries. For example, the Scandinavian researchers adopted different terms compared with the Anglo-American researchers for similar behavioural aggression and thereeir remains no consensus about definitions for the different negative work behaviours (Branch, Ramsay, \& Barker, 2013; Einarsen, \& Nielsen, 2015). In terms of public sector HR in Anglo-American and/or European countries, work harassment has emerged as a negative work behaviour, similar to bullying or mobbing because it is characterised by repeated ill-treatment with negative consequences for the individual (Zapf, Escartin, \& Einarsen, 2011).

Originally, work harassment was conceptualised as part of bullying because of its negative impact on the individual (Raynor, 2002). More recently, work harassment is differentiated from bullying for three main reasons. Firstly, bullying is directed at an individual, whereas work harassment is about task completion and affects large numbers of front-line 
employees facing a chronic problem of demand outstripping supply, forcing them either to choose 'who gets what' (or who misses out) and/or 'to do more with less' on a continual basis (Dick, 2010; Xerri, Farr-Wharton, Brunetto, \& Lambries, 2016). Secondly, bullying can involve physical or mental abuse directed at an individual, whereas work harassment is not personal. Instead, it is the result of a resourcing decision made originally by governments, and then by senior management in allocating resources to each department (Tummers, et al, 2016).

Thirdly, the incidence of work harassment relies on the power of professional principles to ensure patients/clients/customers are serviced, irrespective of organisational resourcing constraints. This is because universities and the professional bodies regulate entry into the professions and work practices are underpinned by specific norms and values (such as professional standards of service and effectiveness) (Farr-Wharton et al., 2011). This in turnoften brings them into conflict with organisational norms (task completion/ reduced per capita costs) leading to 'professional hybridity' (Noordegraaf, 2015). One of the outcomes for healthcare professionals is increasing stress from role conflict as professionals work overtime (often against organisational directions) (Tummers, Vermeeren, Steijn, \& Bekkers, 2012). Hence, work harassment is a type of negative workplace behaviour that is emerging as a growing HR problem, especially for healthcare professionals working in the public sector where austerity dominates resourcing decisions. In particular, Tummers et al. (2016) identified the rise in stress (and stress-related illnesses) emerging amongst professionals because of increasing work harassment, role overload and role ambiguity.

For healthcare workers, high demand is a worldwide phenomenon. In those countries where there is also a shortage of nurses, the workplace conditions are even more likely to enhance, rather than detract from work harassment. For example, Laschinger and Fida (2014) found that in Canada, even new graduate nurses were reporting high-stress levels leading to falling wellbeing and in the worst cases, there was a steady rise in the incidence of high levels 
of burnout and turnover. Laschinger et al. (2014, p19) argue that the key contributors are heavy workloads and inadequate staffing that in turn, create the negative environmental conditions ripe for increasing job dissatisfaction, and:

"Similar to other helping professions, the prevalence of burnout in nursing is particularly high, because of the high emotional and physical demands of this work"

A similar situation was identified for new nurses in the UK, where ethical leaders were identified as a resource countering negative work conditions (Mastracci, 2016). However, it may be the impact of cultural factors intervening for Bangladeshi nurses that negates the impact of work harassment if for example, they perceive the importance of helping pateints are overriding the impact of high workloads on their wellbeing. This is what is unknown - do Bangladeshi employees such as nurses behave similarly to employees in developed countries? In terms of COR theory, high work harassment acts as a negative work behaviour that erodes personal resources. If an employee has a shortage of resources - they will likely see a reduction in their wellbeing. As such, principle two is enacted and consequently, employees seek to control the negative flow of wellbeing by looking for another job (as one example of acting defensively) (Hobfoll 2011). Unless there is a counterbalance of positive resources, for example - positive relationships with their managers that can buffer and negate the impact of work harassment, then employees' personal resources will be negatively affected.

Employee Resources (resource caravans)

1. Manager Support - Leader-member exchange (a social support resource)

Manager-subordinate relationships can be conceptualised via the LeaderMember Exchange (LMX) framework. LMX is a Social exchange theory (SET) variable because the potential benefits from effective workplace relationships far outweigh the initial outlay of good will. LMX argues that managers form different quality relationships with 
employees: for some their workplace interactions are meaningful and over time leads to mutual reciprocity (insiders). On the other hand, for others the interactions are negative and consequently a relationship forms that is built only on the work contract specifications (Martin, Thomas, Guillaume, Lee, \& Epitropaki, 2016). The benefits of an effective LMX relationship were identified in a meta-analysis by Martin et al. (2016) as high role performance and high citizenship behaviour as well as low counteractive workplace behaviour. This relationship becomes even more important when it involves professionals because there is an added responsibility on the part of the manager to ensure the employee is socialised into the values, norms and practices associated with the profession and adheres to professional standards (Brunetto et al., 2018). However, research about public sector nurses in Brazil found that that the theories and "truths" accepted as the behaviour of employees such as nurses in developed countries does not always translate to the behaviour of nurses in BRIC countries. For example, Brunetto, Shacklock, Teo, Farr-Wharton \& Nelson (2015) found that managers had minimal impact on Brazilian nurses' behaviour for numerous reasons, including that there were different contracts in place with some nurses reporting to managers in the capital of Brazil, not the hospital, and the impact of corruption on nurses' actions in the workplace.

However, using COR theory, when LMX is high, it suggests that employees can access support from managers or someone in management. Hence, it is not surprising that previous research in developed countries suggests that high LMX is associated with high wellbeing and low turnover for nurses in Anglo-American and European countries such as Australia, Italy and the UK (Brunetto et al., 2018) as well as the USA (Brunetto et al., 2013). The reason as to why LMX is associated with high employee performance is because as Lashinger and Fida (2014) found in their research of Canadian nurses, high LMX indicates that nurses are receiving high levels of support from managers, which in turn provides a barrier to stress and helps maintain high employee wellbeing. However, the relationship has not been examined for public 
and private sector Bangladeshi nurses, where cultural influences on HR and workplace relationships do not necessarily follow the Anglo-American and/or European model. To test whether similar relationships are in play with Bangladesh for nurses, the following hypotheses are used.

\section{H1. LMX is negatively related to turnover intention}

H2. LMX is positively related to employee wellbeing

Employee well-being (an individual/personal resource)

'Well-being' is a general term with multiple definitions across different disciplines and there is no agreement on a definition except; however, that it is a multi-faceted concept (incorporating physical, job-related, psychological, social wellbeing), and each time it is used, it requires explicit defining in order to avoid confusion (Seligman, 2012). Employee well-being is defined as a composite state that encompasses a hedonic component (life satisfaction) and a eudaimonic component (living life according to one's values) (Forgeard, Jayawickreme, Kern, \& Seligman, 2011) within an occupational context (van der Doef and Maes 1999). Past research argues that high workloads erode nurses' well-being because wellbeing is the barometer indicating how well they are coping (Brunetto et al., 2017). Previous research shows that high wellbeing predicts low turnover for public sector Australian nurses (Brunetto, Rodwell, Shacklock, Farr-Wharton, \& Demir, 2016a) and high work harassment is associated with low wellbeing for Australian and Italian public and private sector nurses (Brunetto et al., 2016b).

\section{Turnover Intentions}

Turnover intentions refers to employees' propensity to withdraw from their present workplace and consequently, they conserve their energy at work and redirect it towards looking at alternatives (Van Knippenberg, Van Dick, \& And Tavares, 2007). There are multiple push factors likely to promote turnover intentions, including poor quality relationships and the lack of support given to staff (Van Knippenberg et al, 2007; Lashinger \& Fida, 2014) as well as low 
wellbeing for US and Australian nurses (Brunetto et al., 2013). In each case, high wellbeing was associated with low turnover intentions. The following hypotheses replicate this finding for Bangladeshi nurses.

\section{H3. Employee wellbeing is negatively related to turnover intention}

\section{Mediation and Moderations}

The way that work harassment and wellbeing impact the relationship between LMX and turnover for Bangladeshi public and private sectors is not clear. There are two types of possible effects. First, work harassment moderates the association between LMX and wellbeing, and between LMX and turnover intentions. If this is the case then it means that work harassment affects the direction or strength of the relationship between LMX and wellbeing, and LMX and turnover intentions (Baron \& Kenny, 1986). Second, wellbeing mediates the association between LMX and turnover intentions, which means that employee wellbeing accounts for some of the negative relationship between LMX and turnover intentions. The two types of effects will be discussed further in the following paragraphs.

In terms of COR theory, wellbeing is the measure of an individual's personal resources and is affected by both personal (such as psychological capital) and organisational (such as LMX) factors. In particular, high LMX is associated with high wellbeing for Australian public sector nurses (Brunetto et al., 2016). In addition, high wellbeing is associated with low turnover intentions for Australian and US nurses (Brunetto et al., 2013). This means that if employees have high wellbeing (perhaps because of high personal resources), it may mediate the relationship between LMX and turnover intentions. According to COR theory, if wellbeing is high enough, we expect that the personal resources gained from high wellbeing will mediate the impact of LMX on turnover. The following hypothesis expresses this premise. 
Moderation refers to a situation where the relationship between two variables depends on a third variable. As stated, previous research shows that high LMX is associated with high wellbeing and high work harassment is associated with low wellbeing. In the context of Bangladeshi nurses, we propose that high work harassment results in employees feeling overworked, under-resourced, and that overall their employing organisation's expectations are unrealistic. In such a work environment, even if employees have a good relationship with their manager, it is expected that high work harassment will reduce the positive impact of LMX and employee wellbeing, and decrease the negative impact of LMX on turnover intention. Moreover, we expect that work harassment will moderate the impact of LMX because a manager is considered an agent of the organisation (Eisenhardt, 1989), and thus unrealistic expectations may flow through the manager to the nurses. Therefore, if a nurse thinks expectations are unrealistic, they may associate such expectations with the manager, which when work harassment is high will reduce the positive influence of the manager on employee wellbeing and reduce the negative affect on turnover intentions.

\section{H5. Work harassment moderates the relationship between LMX and wellbeing}

\section{H6. Work harassment moderates the relations between LMX and turnover intentions}

\section{The public-private context}

The context of this research is a comparison of the public and private sector work environment for Bangladeshi nurses. The public sector in Bangladesh is highly bureaucratic and hierarchical, and is described as being over-governed (micro-managed and autocratic) with insufficient or inappropriate governance (subject to corruption) (Ahmed \& Shirahada, 2017). This means that there is a lack of separation between the official practices of public sector officials and personal interests, and a growing realisation that recent reforms, whilst in theory about re-distributing resources from the rich to the poor, did not achieve the stated goals (Khan 
et al, 2014). Also despite specific legislation promoting equal rights for women, reforms have not made much of an indentation on the glass ceiling imposed upon women by the dominant culture in play (Jamil, Aminuzzaman \& Hoque, 2013). According to Ahmad et al. (2007) (cited in Ghuman \& Singh, 2013) reforms were supposed to provide greater access to healthcare for the poor. However, in practice, the rich were given greater access to resources, and nurses were dissuaded against going to poorer regions because of inadequate infrastructure facilities. In summary, reforms did not achieve a greater focus on efficiency or achieving outcomes. Hence, LMX, work harassment, wellbeing and turnover is expected to be worse for public sector nurses. In the other hand, Farr-Wharton et al (2019) found work harassment was also prevalent for UK and US private sector nurses. The hypothesis is:

H7: Private sector employees perceive higher LMX and wellbeing and lower work harassment and turnover compared with public sector nurses.

\section{Method}

Sample and procedure

In the first instance, nurses who had completed an undergraduate and/or postgraduate nursing qualification from the Grameen Caledonian College of Nursing, Dacca, Bangladesh were invited to complete the survey. In total, 191 useable surveys were returned (redemption rate of $62 \%$ ). Additionally, 126 useable surveys were received from nurses working in two public sector hospitals in Dacca, Bangladesh. The data was collected in 2017 and 2018.

In total, 317 useable surveys were obtained, 131 from the public sector and 186 from the private sector. The demographic data depicts that unlike the ageing nursing workforce in many OECD countries (World Health Organization, 2018); the majority of nurses sampled in the Bangladesh public and private sectors were less than 31 years of age. As well, many have less than one-year employment with their current hospital, with the majority having less than 
5 years employment in their current hospital. In the public sector, there were 34.4 per cent males $(n=45)$ and 65.6 per cent females $(n=86)$, which is a stark comparison to the private sector with 4.3 per cent males $(n=8)$ and 95.6 per cent females $(n=178)$.

\section{Measures}

All items for each scale were scored on a six-point Likert scale from 1 (strongly disagree) to 6 (strongly agree). The reliability for each scale is depicted in Table 1 .

Leader-member exchange. To measure a nurse's relationship with their line manager, we used the Leader-Member Exchange (LMX) instrument developed by Graen and Uhl-Bien (1995). An example of an item is 'My manager is satisfied with my work.'

Employee wellbeing. We measured an employee's sense of their wellbeing with the four items from Brunetto, Farr-Wharton, and Shacklock (2011). A sample item includes 'Overall, I am reasonably happy with my work life.'

Turnover intentions. To identify nurse intentions to leave their employing organisation, we used three-items adopted from Meyer, Allen, and Smith (1993) scale of turnover intentions, including, 'I frequently think about leaving my current employer.'

Work harassment. Rayner and Cooper (2006) developed a scale to measure task-attack, personal-attack and intimidation. To measure work harassment, we used three items that examine 'task-directed attack,' including the item '[This hospital] sets unrealistic targets.'

Control variables. Many variables within the nursing and turnover intention literature may impact turnover intentions. We controlled for tenure within a hospital because research has found those with longer tenure have a lower intention to leave their employing organisation (Ng \& Feldman, 2009b). Although there is limited agreement in the literature, we also controlled for gender, with one study suggesting women quit their job less than their male counterparts (e.g., Lyness \& Judiesch, 2001), and a larger scale study of 475,000 professionals and managers concluding women were more likely to voluntarily leave. Finally, we controlled 
for age because of $\mathrm{Ng}$ and Feldman's (2009a) postulation that older employees typically develop stronger ties to their colleagues and their employing organisations.

\section{Data analysis}

To test a moderated mediation model, we used SPSS v.25 and the PROCESS Macro by Andrew Hayes (Hayes, 2018). The PROCESS macro is a better alternative to test mediation when compared to approaches such as that prescribed by Baron and Kenny (1986), as there is no requirement for the data to be normally distributed. Within the PROCESS macro, we used model 8 and applied 95 per cent bootstrap confidence intervals across a sample of 5000. To confirm the factor structure and to account for common method variance, we undertook a confirmatory factor analysis of the public and private sectors. To check and control for common method variance, we included a common latent factor (Podsakoff, Mackenzie, Lee, \& Podsakoff, 2003).

\section{Results}

\section{Descriptive statistics and intercorrelations}

Table 1 depicts the descriptive statistics, scale reliability and intercorrelations. Notably, LMX did not significantly influence turnover intentions in the public sector. Although not significant, the $p$-value was nearing statistical significance $(p=0.06)$.

\section{Confirmatory factor analysis}

The hypothesised measurement model resulted in a poor fitting model for the private $(\mathrm{CMIN} / \mathrm{DF}=2.330, \mathrm{RMSEA}=0.085, \mathrm{CFI}=0.886$ and $\mathrm{SRMR}=0.066)$ and public $(\mathrm{CMIN} / \mathrm{DF}$ $=3.222, \mathrm{RMSEA}=0.131, \mathrm{CFI}=0.857$ and SRMR $=0.081)$ sectors. Due to factor loadings below 0.5 , a number of observed items were removed. We removed three LMX questions: 1. 'Regardless of how much formal authority he/she has built into his/her position, what are the chances that your leader would use his/her power to help you solve problems 
in your work? (What are the changes that you would)'; 2. 'have enough confidence in my leader that I would defend and justify his/her decision if he/she were not present to do so?'; and 3. 'How would you characterize your working relationship with your leader?'. We also removed one wellbeing item, 'I get enough time to reflect on what I do in the workplace'. The modified measurement model fit the data well for the private $(\mathrm{CMIN} / \mathrm{DF}=1.89, \mathrm{RMSEA}=.069, \mathrm{CFI}=$ .945 and $\mathrm{SRMR}=.058)$ and public $(\mathrm{CMIN} / \mathrm{DF}=1.98, \mathrm{RMSEA}=.087, \mathrm{CFI}=.953$ and $\mathrm{SRMR}$ $=.067)$ sectors. We tested a number of alternate models to establish the distinctiveness of the variables and the modified measurement model. As depicted in Table 2, the fit of the alternate models was significantly worse when compared with the modified measurement model for the private and public sectors.

The addition of a common latent factor (CLF) to the modified measurement model (model 4) for the private and public sectors resulted in improved model fit. In addition, a chisquare difference test for each sample revealed that the CLF model was statically different from the modified measurement model, and thus common method variance may explain a significant portion of the variance. To identify where common method variance was problematic, we compared the standardised factor loadings of the modified measurement model and the CLF model. To the best knowledge of the authors, there are no published cut-off values for the comparison of standardised factor loadings. We considered any loading difference above 0.20 to be excessively large and retained the CLF control. The final measurement model for the private and public sector include the bias-corrected estimates (i.e., controlling for common method bias). Specifically, to be able to test the hypotheses within the PROCESS macro, we imputed the bias-correct composite variables from AMOS into SPSS.

Testing the hypotheses

Figure 1 depicts the results for hypotheses 1-3 for the public and private sector. LMX predicts turnover $(\mathrm{H} 1)$ for private sector employees only and LMX predicts wellbeing $(\mathrm{H} 2)$ for 
both public and private sector and wellbeing predicts turnover intentions (H3) for public sector employees only. There was support for the acceptance of hypothesis 4 (mediation), with a significant indirect effect $\mathrm{SE}=-.21, \mathrm{CI}(-.42,-.10)$. As mentioned, the direct effect between LMX and turnover intentions was not significant, providing support that wellbeing completely mediates the relationship between LMX and nurse turnover intentions in the Bangladesh public sector. We also found work harassment did not moderate the relationship between LMX and turnover intentions (i.e., rejecting hypothesis 6), but, as depicted in Figure 2, work harassment did moderate the path between LMX and employee wellbeing (i.e., providing support for the acceptance of hypothesis 5). Specifically, high work harassment reduced the influence of LMX on employee wellbeing.

In relation to the private sector, wellbeing did not directly relate to turnover intentions, so we rejected hypothesis 2 . Due to the rejection of hypothesis 3 , and insignificant indirect effects, we rejected hypothesis 4 . Finally, work harassment did not moderate the relationship between LMX and wellbeing, or LMX and turnover intentions. As such, we rejected hypotheses 5 and 6 .

\section{Discussion}

This paper examines the impact of line management on work harassment, wellbeing and turnover of public and private nurses in Bangladesh using a COR framework. One factor which has been identified as having a negative impact, especially in the public sector, is work harassment and in developed countries, the manager either alleviates the situation or makes it worse (Brunetto et al, 2016b). In contrast, Farr-Wharton et al (2019) also identified work harassment for private sector nurses in the UK and USA. However, research in Brazil found that management did not have the same impact compared with Anglo-American and/or European countries such as UK and Australia (Brunetto et al, 2015), hence, we examined the impact of management on public and private nurses' perceptions of work harassment in 
Bangladesh. Additionally, since, private sector nurses operate in hospitals that have attempted to implement some typically western HR systems, it seems likely that turnover should be less because the influence of culture (corruption etc) should be less.

In terms of the first and second hypotheses, previous research had already shown that effective management is associated with high wellbeing and low turnover intentions for public and private sector employees in Anglo-American and European countries (Martin et al., 2016; Brunetto et al., 2018, 2013). The findings from this research support previous research showing that high LMX is associated with high wellbeing for both public and private sector nurses in Bangladesh. The role of the line manager is to act as a source of support when workloads are physically and mentally draining - hence in the ideal situation, they provide a barrier to stress, in turn supporting high wellbeing (Lashinger \& Fida, 2014). However, high LMX is only associated with low turnover for private sector nurses in Bangladesh. One explanation is that public sector nurses are unlikely to have a modern HR system in place (of which a basic manager-employee relationship is an important cornerstone), and instead, the traditional culture may be more important in determining whether employees stay or leave (Ahmed \& Shirahada, 2017; Khan et al., 2014). Khan et al (2014) argues that in Bangladesh, the policy may be similar to a developed country, but the implementation of HR policies has been challenging, especially in the public sector. Hence, it seems likely that family ties and corruption still dominate decision making, in turn negating the role of managers.

Additions, the findings suggest that in contrast to the role of line managers of professionals in Anglo-American and European countries, who play two distinct (sometimescontradictory) roles - professional hybridity, firstly developing nurses' professional skills and knowledge, while still acting as their line manager (Noordegraaf, 2015); the role of public sector line managers in Bangladesh is unclear because nurses' wellbeing is determined by how work harassment mediates the relationship between LMX and wellbeing. One possible reason 
is that their managers are not engaging in professional activities such as socialising them into the professional norms and values associated with nursing. Khan et al (2014) suggests that in countries like Bangladesh, increased professionalization of the Bangladeshi public sector has failed to change 'who gets what' and how workplaces are organised (Jamil, 2013). Hence LMX may not be based on professional factors and the managers may not act as positive antecedents of nurses' wellbeing as is the case in developed countries (Martin et al, 2016). However, further research should examine why a significant relationship was absent.

Regarding hypothesis 3, previous research had already shown that high wellbeing was associated with low turnover intentions for nurses in the USA and Australia (Brunetto et al., 2016). In terms of COR theory, high wellbeing is an important personal resource that employees want to develop, and conserve when required (Hobfoll 2011). The findings from this research are consistent with past research and COR theory for public sector Bangladeshi nurses only. More research is required to investigate the relationship for private sector nurses. It may be that the traditional culture has a role to play in better understanding the relationship between wellbeing and turnover for Bangladeshi nurses working in the private sector (Khan et al., 2014). Such insight may better explain how employees build wellbeing in the workplace, and importantly, why they stay even in workplace enrivonments that deplete their wellbeing.

The mediation and moderation processes shed further light on understanding the differences in the public and private workplaces for nurses in Bangladesh. Past research shows that work harassment is a product of the era of austerity, which now governs the work lives of many professionals in the caring industry, especially in the public sector in developed countries. However, very little is known about how reforms (if any?) have affected the professional workplace in developing countries. In ideal circumstances, professionals expect their line managers to mediate unrealistic organisational work demands and provide appropriate and sometimes-extra support resources to ensure both task completion and professional 
development occurs (Farr-Wharton et al., 2011). In this study, work harassment does mediate the relationship between LMX and wellbeing for public sector nurses, but not for private sector nurses. Over half of public sector Bangladeshi nurses' wellbeing is explained by how work harassment mediates the relationship between LMX and wellbeing.

While previous research shows that LMX predicts work harassment (Farr-Wharton et al., 2018), and work harassment predicts employees' wellbeing (Xerri et al., 2016), this paper adds new evidence about the role of work harassment in moderating the relationship between management and wellbeing of public sector nurses in Bangladesh. More specifically, in the public sector, when work harassment was low, management had a greater positive influence on employee wellbeing. These results provide new evidence about the influence of work harassment on the well-established association between management practices and wellbeing. This means that the role of the manager is just as important in developing countries such as Bangladesh as it is in developed countries, although culture may affect how it works. It also aligns with COR theory in that those with fewer resources are less able to apply resources to develop other resources, which suggests that those managers who support their nurses impact their decision to stay by improving their wellbeing. However, this is the case for public sector nurses only.

In contrast, LMX explains just under a quarter of private sector nurse wellbeing, and work harassment has no significant impact on this association. Further, there is no evidence of work harassment moderating the relationship between LMX and turnover in this study, although, high LMX, work harassment and wellbeing does explain just under a third of the turnover intentions of public sector Bangladeshi nurses. In the case of private sector Bangladeshi nurses, work harassment alone explains well over a third of their turnover intentions. This contrasts research by Farr-Wharton et al (2019) about US and UK private

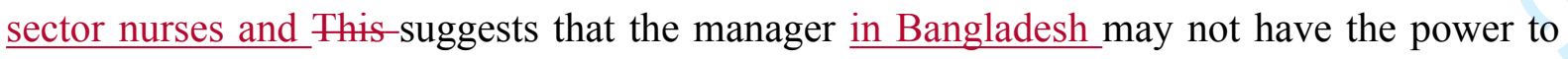


affect how work harassment influences turnover intentions for private sector nurses. Hence these This is in contrast to research findings differ from those in the Anglo-American and/or European countries which may suggest a significantly different work context for these private sector nurses working in one of the least developed countries in the world. The findings provide a platform for more research about management issues facing public and private professionals such as nurses in developing countries such as Bangladesh.

The last hypothesis examined the differences between the public and private sector nurses' level of satisfaction with LMX, work harassment, wellbeing and turnover. The belief was that private sector nurses would perceive higher LMX and wellbeing and lower work harassment and turnover intentions compared to their private sector counterparts. The hypothesis was based on information that public sector reforms have not been successfully implemented, the public sector in Bangladesh is poorly resourced, and nurses are actively dissuaded from going to poorer regions because of inadequate infrastructure facilities (Jamil, 2013). However, the hypothesis was rejected because, across every factor, public sector nurses were more satisfied with management, had higher wellbeing, perceived lower work harassment and turnover intentions (See Table 1). One explanation using COR theory is that private sector nurses' wellbeing is being conserved and developed from other resources not examined in this paper. Future studies should further examine the antecedents, mediators and moderators relating to work harassment.

In reviewing this study, there are some limitations to consider. First, the cross-sectional data was susceptible to common method bias (Podsakoff et al., 2003). However, as mentioned, a common latent factor was included, and several items that were explained by common method variance were controlled, as per George and Pandey's recommendations (2017). Nonetheless, future research should examine work harassment over time to improve insight and design relating to common method bias. Second, although a study on Bangladeshi nurses 
provides some insight into work harassment in developing countries, further research is required about other developing countries. Further, another limitation relates to the sample in that most of the nurses had worked less than five years in their current employment, and therefore, there may be other factors, other than LMX and wellbeing affecting their intention to stay that have not been examined in this paper. Finally, the findings are limited by the sample size for each sector, and the type of demographic information collected that could have assisted in explaining it. Further research should be undertaken to increase the sample size, which will improve the stability of the results, and provide enough statistical power to examine more complex research models, including a thourough examination of work harassment antecedents, mediators and moderators.

\section{Conclusion}

The findings suggest that work harassment is prevalent in the Bangladesh public and private nursing profession. This study used a COR theoretical framework to compare the impact of management practices (LMX) and work harassment on the wellbeing and turnover intentions of Bangladeshi public and private nurses. Using COR theory, we found that LMX is a positive resource caravan passageway that can build wellbeing for public sector employees; however, the association was not significant for private sector nurses. Hence, COR theory principle 2 offers one explanation of $r$ public sector employees' behaviour in that it assumes that the manager provided enough of a 'cushion' for them to form a barrier against the negative influence of work harassment on their wellbeing (Hobfoll, 2001). Similar to per past AngloAmerican and European research has shown, the consequences of poor environmental conditions, such as work harassment, is that it does affect how public sector nurses in Bangladesh are able to take advantage of the relationship with their manager. However, there was no evidence that employees who perceive high support from their line managers are less 
likely to be negatively influenced by work harassment (Hobfoll, 2011)..Therefore, whilst COR theory was somewhat useful in explain some behaviour of public sector Bangladeshi nurses, it was far less relevant for explaining the behaviour of those in the private sector. This is an important finding because theories and concepts developed and validated in Anglo-American contexts assume underlying cultural similarities that may not be accurate in developing countries. Future research should perhaps test other well established theories (and concepts) across multiple contexts to verify their validity.

The implication of this research relates directly to public sector management. For example, the impact of management is different in Bangladesh compared with past AngloAmerican and European studies, and one factor is likely to be the traditional cultural stronghold, especially the impact of ties and corruption, which affects nurses' turnover intention, especially in public sector nurses areas (Ahmed \& Shirahada, 2017). Future research should explore those assertions. Additionally, there are significant differences in how public and private sector nurses respond to, and cope with work harassment. Hence, simply transplanting management models from developed countries for managing healthcare in developing countries may not yield its intended benefits. More research is required to better understand how managers affect how professionals work in developing countries. 


\section{References}

Ahmed, T \& Shirahada, K (2017) Toward a sustainable healthcare service system in a limited resource context: Case study of Bangladesh rural advancement committee's healthcare system, International Journal of Healthcare Management,

DOI: $10.1080 / 20479700.2017 .1389512$

Baron, R. M., \& Kenny, D. A. (1986). The moderator-mediator variable distinction in social psychological research: Conceptual, strategic, and statistical considerations. Journal of Personality and Social Psychology, 51(6), 1173-1182.

Branch, S., Ramsay, S., \& Barker, M. (2013). Workplace bullying, mobbing and general harassment: A review. International Journal of Management Reviews, 15, 280-299

Brunetto, Y., Farr-Wharton, R., \& Shacklock, K. (2011). Using the Harvard HRM model to conceptualise the impact of changes to supervision upon HRM outcomes for different types of public sector employees International Journal of Human Resource Management 22(3), 553-573.

Brunetto, Y., Rodwell, J., Shacklock, K., Farr-Wharton, R., \& Demir, D. (2016a). The impact of individual and organizational resources on nurse outcomes and intent to quit. Journal of advanced nursing, 72(12), 3093-3103.

Brunetto, Y., Xerri, M., Shriberg, A., Farr-Wharton, R., Shacklock, K., Newman, S., \& Dienger, J. (2013). The impact of workplace relationships upon engagement, wellbeing, commitment and turnover for nurses in Australia and USA. Journal of advanced nursing, 69(12), 27862799.

Brunetto, Y., Xerri, M., Trinchero, E., Beattie, R., Shacklock, K., Farr-Wharton, R., \& Borgonovi, E. (2018). Comparing the impact of management on public and private sector nurses in the UK, Italy, and Australia. Public Management Review, 20(4), 525-544.

Brunetto, Y., Xerri, M., Trinchero, E., Farr-Wharton, R., Shacklock, K., \& Borgonovi, E. (2016b). Public-Private Sector Comparisons Of Nurses' Work Harassment Using Set: Italy and Australia. Public Management Review, 18(10), 1479-1503. doi:DOI:10.1080/14719037.2015.1114136

Brunetto, Y., Shacklock, K., Teo, S., Farr-Wharton, R., \& Nelson, S. (2015). Nurses' managers, learning options and organisational commitment: Australia, Brazil and England. Journal of Nursing Management. $23(8), 1029-1038$

Dick, G. P. (2010). Can the organisation and supervision environment influence both bullying and organisational commitment? Evidence from a police force survey. Kent University Working Paper Series 196.

Diefenbach, T. (2009). New public management in public sector organizations: the dark sides of managerialistic 'enlightenment'. Public Administration, 87(4), 892-909.

Einarsen, S. (2000). Harassment and bullying at work: A review of the scandinavian approach. Aggression and Violent Behavior, 5(4): 379-401

Einarsen, S., Hoel, H., \& D. Zapf, D. eds. (2011). Bullying and Harassment in the Workplace: Developments in Theory, Research and Practice ( $2^{\text {nd }}$ Edition). CRC Press, Taylor \& Francis Group, Boca Raton, Florida.

Einarsen, S., \& Nielsen, M. (2015). Workplace bullying as an antecedent of mental health problems: A five-year prospective and representative study. International Archives of Occupational and Environmental Health. 88: 131-142

Eisenhardt, K. M. (1989). Agency theory: An assessment and review. Academy of Management Review, 14(1), 57-74.

Farr-Wharton, R., Brunetto, Y., \& Shacklock, K. (2011). Professionals' manager-subordinate relationships, autonomy and commitment in Australia: a leader-member exchange theory perspective. The International Journal of Human Resource Management, 22(17), 3496-3512. 
Forgeard, M. J., Jayawickreme, E., Kern, M. L., \& Seligman, M. E. (2011). Doing the right thing: Measuring wellbeing for public policy. International journal of wellbeing, 1(1), 79-106.

George, B., \& Pandey, S. (2017). We know the Yin, but where is the Yang? Toward a balanced approach on common source bias in public administration scholarship. Review of Public Personnel Administration, 37(2), 245-270

Ghuman, B. S., \& Singh, R. (2013). Decentralization and delivery of public services in Asia. Policy and Society, 32, 7-21

Graen, G., \& Uhl-Bien, M. (1995). Relationship-based approach to leadership: Development of leader-member exchange (LMX) theory of theory over 25 years: Applying a multi-level multidomain perspective. Leadership Quarterly, 6(2), 219-247.

Halbesleben, J. R., Neveu, J.-P., Paustian-Underdahl, S. C., \& Westman, M. (2014). Getting to the "COR" understanding the role of resources in conservation of resources theory. Journal of management, 40(5), 1334-1364.

Hayes, A. F. (2018). Introduction to mediation, moderation, and conditional process analysis: A regression-based approach. New York: Guilford Press.

Hobfoll, S. E. (1989). Conservation of resources: A new attempt at conceptualizing stress. American psychologist, 44(3), 513-524.

Hobfoll, S. E. (2001). The influence of culture, community, and the nested-self in the stress process: advancing conservation of resources theory. Applied psychology, 50(3), 337-421.

Hobfoll, S. E. (2011). Conservation of resource caravans and engaged settings. Journal of occupational and organizational psychology, 84(1), 116-122.

Jamil, I., Aminuzzaman, S., \& Haque, S. (2013) Introduction: Governance in South, Southeast, and East Asia Public Organization Review 13 (4) 341- 347

Khan., R, Miah., M, \& Manzoor., A (2014). Human Resource Management Practices: A Case Study of South Asian Countries IBT Journal of Business Studies 9(1), 83-101

Lyness, K. S., \& Judiesch, M. K. (2001). Are female managers quitters? The relationships of gender, promotions, and family leaves of absence to voluntary turnover. Journal of applied Psychology, 86(6), 1167-1178.

Mahdy, H. A. (2009) Reforming the Bangladesh healthcare system, International Journal of Health Care Quality Assurance, 22(4), 411-416.

Martin, R., Thomas, G., Guillaume, Y., Lee, A. \& Epitropaki, O. (2016). Leader-member Exchange

(LMX) and Performance: A Meta-analytic Review. Personnel Psychology, 69, 67-121.

Meyer, J. P., Allen, N., \& Smith, C. A. (1993). Commitment to organizations and occupations: extension and test of a three-component conceptualization. Journal of applied Psychology, 78(4), 538-551.

Mastracci, S. (2016). Beginning nurses' perceptions of ethical leadership in the shadow of Mid Staffs. Public Integrity, 19(3), 250-264.

Ng, T. W., \& Feldman, D. C. (2009a). Age, work experience, and the psychological contract. Journal of Organizational Behavior, 30(8), 1053-1075.

$\mathrm{Ng}, \mathrm{T}$. W., \& Feldman, D. C. (2009b). Re-examining the relationship between age and voluntary turnover. Journal of Vocational Behavior, 74(3), 283-294.

Noordegraaf, M. (2015) Hybrid Professionalism and Beyond: (New) Forms of Public Professionalism in Changing Organizational and Societal Contexts, Journal of Professions and Organization, 2, 187-206.

Podsakoff, P. M., MacKenzie, S. B., Lee, J.-Y., \& Podsakoff, N. P. (2003). Common method biases in behavioral research: a critical review of the literature and recommended remedies. Journal of applied Psychology, 88(5), 879-903.

Rayner, C., \& Cooper, C. L. (2006). Workplace bullying. In K. E. Kelloway, J. Barling, \& J. J. H. Jr. (Eds.), Handbook of workplace violence. Thousand Oaks: Sage Publications.

Laschinger, H.K.S., Fida, R., (2014). New nurses' burnout and workplace wellbeing: the influence of authentic leadership and psychological capital. Burnout Research 1 (1), 19-28 
Seligman, M.E., (2012). Flourish: A visionary new understanding of happiness and well-being. New York: Simon and Schuster.

Tummers, L., Vermeeren, B., Steijn, B., \& Bekkers, V. (2012). Public professionals and policy implementation: Conceptualizing and measuring three types of role conflicts. Public Management Review, 14(8), 1041-1059.

Tummers, L., Brunetto, Y., Teo, S., (2016) Workplace aggression: Introduction to the special issue and future research directions for scholars, International Journal of Public Sector Management, 29 (1), $2-10$

Van Knippenberg, D., Van Dick, R., \& and Tavares, S. (2007). Social Identity and Social Exchange: Identification, Support, and Withdrawal from the Job. Journal of Applied Social Psychology, 37(3), 457-477.

Van der Doef, M. and Maes, S., 1999. The job demand-control (-support) model and psychological well-being: a review of 20 years of empirical research. Work \& stress, 13(2), pp.87-114.

World Bank. (2016). Overview of Bangladesh. Retrieved from http://www.worldbank.org/en/country/bangladesh/overview

World Health Organization. (2016). World Health Statistics 2016: Monitoring Health for the Sustainable Development Goals (SDGs). World Health Organization. Retrieved from http://www.who.int/gho/publications/world health statistics/2016/en/

World Health Organization. (2018). Nursing and Midwifery Fact Sheet February 2018. Retrieved from http://www.who.int/mediacentre/factsheets/nursing-midwifery/en/

Xerri, M., Farr-Wharton, R., Brunetto, Y., \& Lambries, D. (2016). Work harassment and local government employees: Australia and USA. International Journal of Public Sector Management, 29(1), 54-71.

Zapf, D., Escartin,J., Einarsen, S., Hoel, H. \& Vartia, M. (2011). Empirical findings on the prevalence and risk groups of bullying in the workplace. In Bullying in the Workplace; Developments in Theory, Research and Practice edited by Einarsen, S., Hoel, H., Zapf, D., Cooper. C. CRC press, Taylor \& Francis group, Boca Rata 
Table 1. Correlation matrix

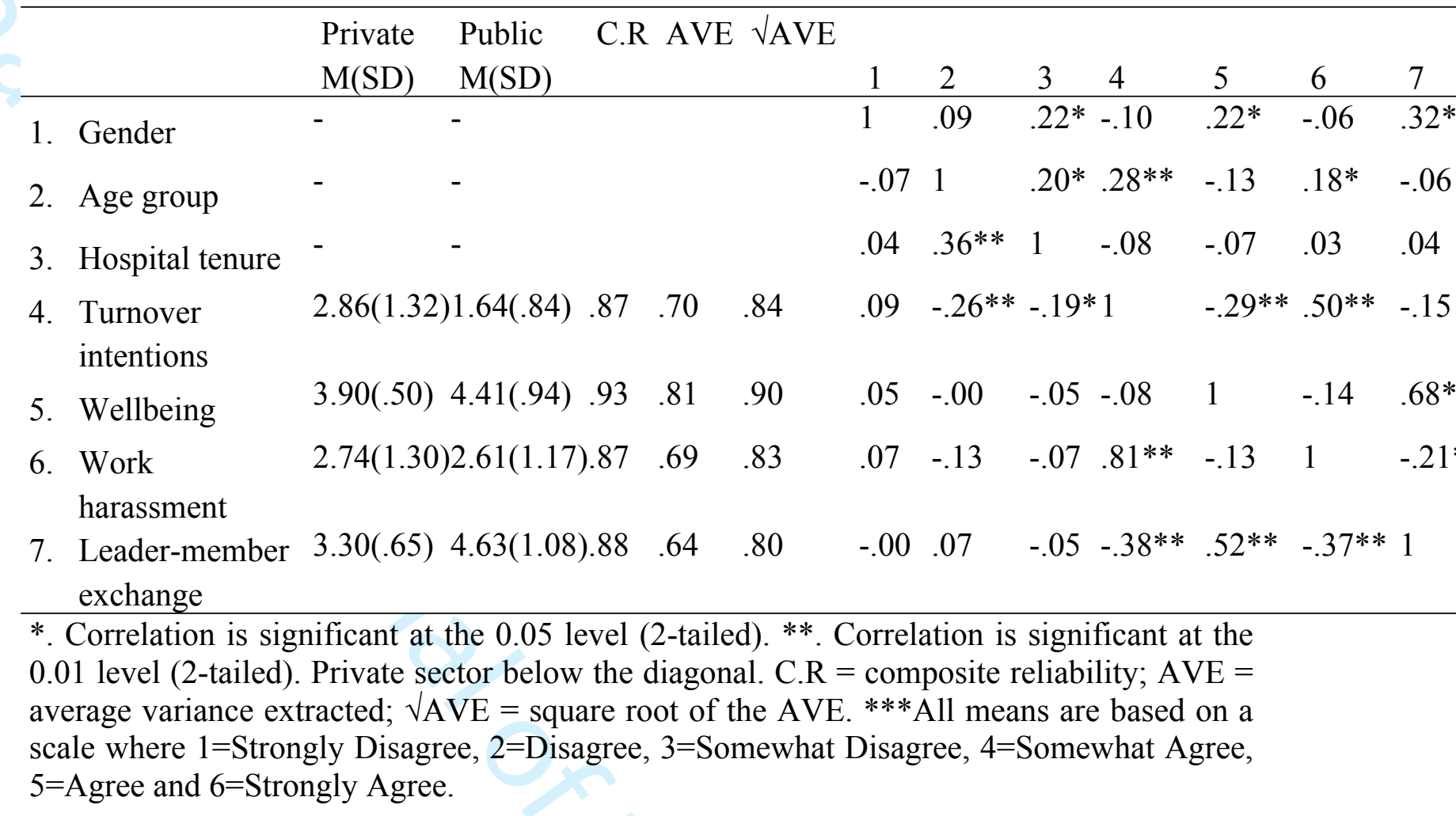

Table 2. Comparison of alternate measurement models

\begin{tabular}{|c|c|c|c|c|c|c|c|}
\hline Model & & $\chi^{2}$ & $\Delta \chi^{2}$ & df & $\Delta \mathbf{d f}(p)$ & CFI & SRMR \\
\hline & $\begin{array}{l}\text { Hypothesised measurement model } \\
\text { Private sector }\end{array}$ & 265.217 & & 113 & & .886 & .066 \\
\hline Base & $\begin{array}{l}\text { Modified measurement model - private } \\
\text { sector }\end{array}$ & 111.608 & & 59 & & .945 & .058 \\
\hline 1 & Combine LMX and wellbeing & 181.715 & 70.11 & 62 & $3(\mathrm{p}<.001)$ & .874 & .084 \\
\hline 2 & Combine LMX and Harassment & 317.966 & 206.36 & 62 & $3(\mathrm{p}<.001)$ & .731 & .146 \\
\hline 3 & Combine wellbeing and harassment & 229.781 & 118.7 & 62 & $3(\mathrm{p}<.001)$ & .824 & .118 \\
\hline \multirow[t]{2}{*}{4} & Add CLF to Base model & 76.394 & 35.21 & 46 & $13(\mathrm{p}<.001)$ & .968 & .045 \\
\hline & $\begin{array}{l}\text { Hypothesised measurement model (four } \\
\text { factor model) - Public sector }\end{array}$ & 364.098 & & 113 & & .857 & .081 \\
\hline Base & $\begin{array}{l}\text { Modified measurement model - public } \\
\text { sector }\end{array}$ & 116.799 & & 59 & & .953 & .067 \\
\hline 1 & Combine LMX and wellbeing & 309.179 & 192.38 & 62 & 3( & .797 & .090 \\
\hline 2 & Combine LMX and Harassment & 326.026 & 209.23 & 62 & $3(\mathrm{p}<.001)$ & .783 & .161 \\
\hline 3 & Combine wellbeing and harassment & 332.380 & 215.58 & 62 & $3(\mathrm{p}<.001)$ & .778 & .164 \\
\hline 4 & Add CLF to Base model & 69.596 & 42.203 & 46 & $13(\mathrm{p}<.001)$ & .981 & .046 \\
\hline
\end{tabular}




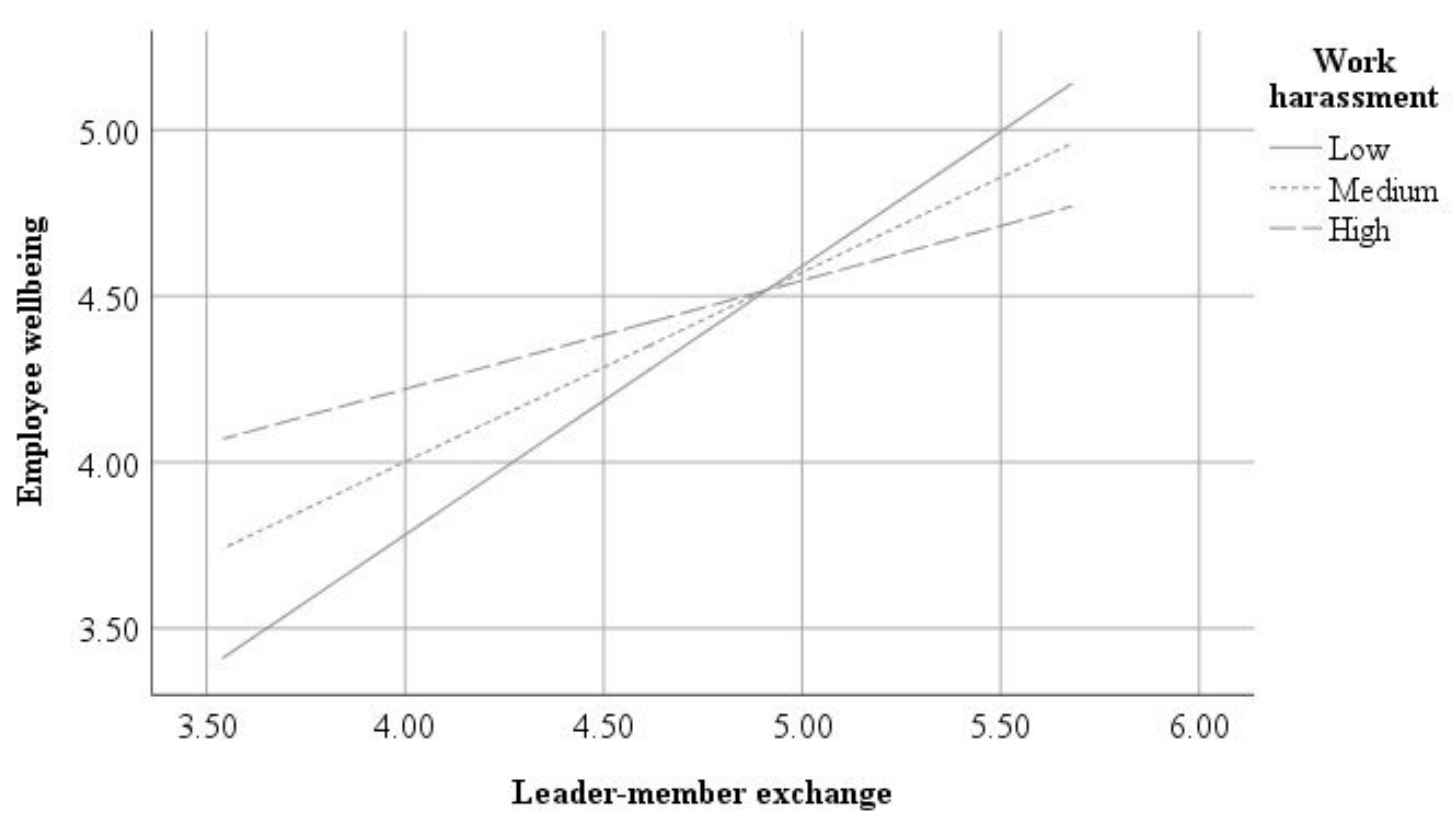

Figure 2. Moderation results - work harassment moderates the relationship between LMX and employee wellbeing. Note: Work harassment standardised for moderation analysis 


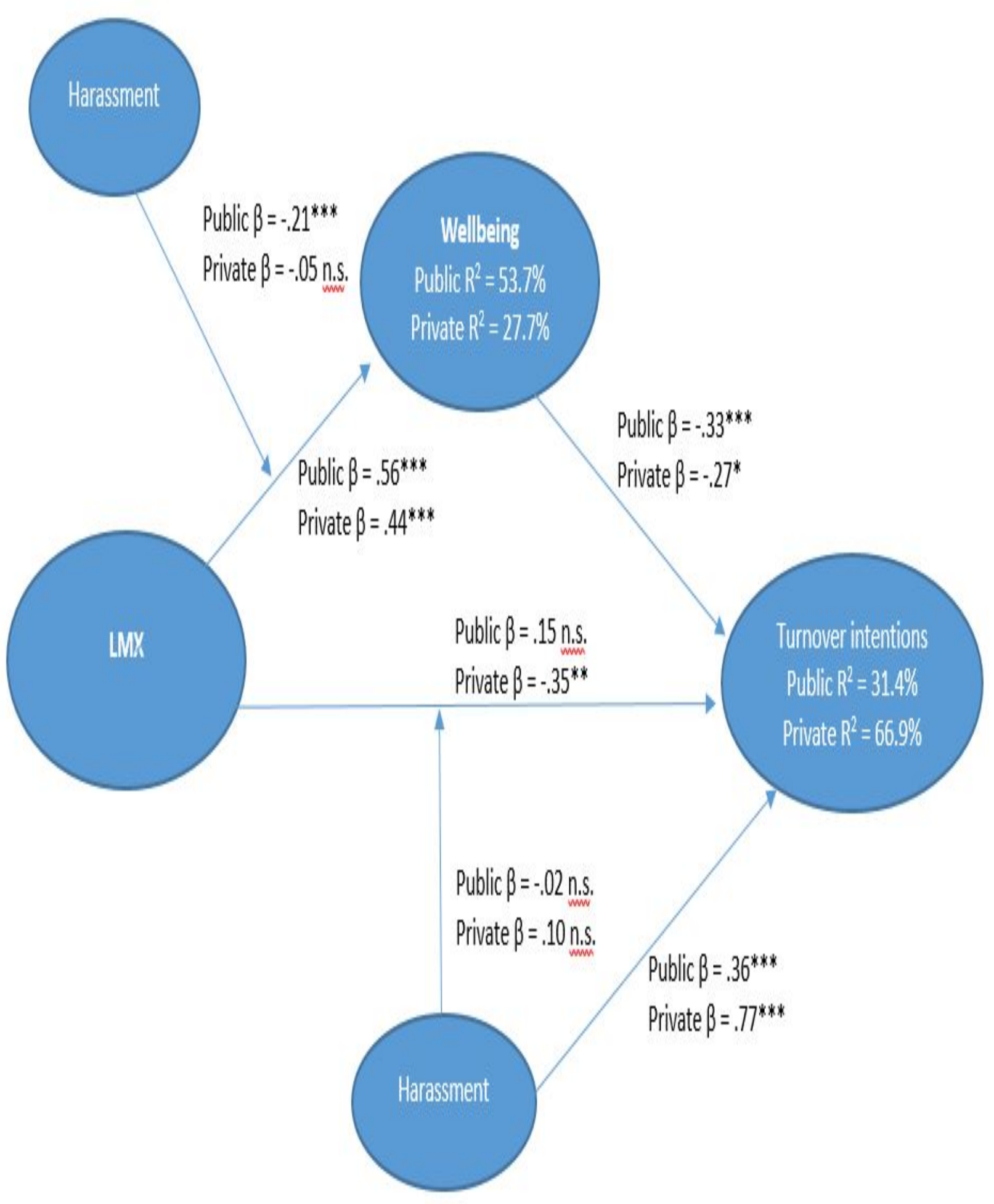




\title{
Comparing the impact of management on public and private nurses in Bangladesh
}

\begin{abstract}
Purpose: This paper uses Conservation of Resources (COR) theory as a lens for comparing the impact of line management on Bangladeshi public and private nurses' perception of work harassment, wellbeing and turnover intentions where Anglo-American and European management models have been super-imposed on an existing different culture.
\end{abstract}

Design: Survey data was collected from 317 Bangladeshi nurses' (131 from the public sector and 186 from the private sector). Structural Equation Modelling was used for analysis.

Findings: High work harassment was associated with low wellbeing and management practices, and explained approximately a quarter of private sector nurses' wellbeing. In total, management, work harassment and employee wellbeing explained approximately a third of the turnover intentions of public sector nurses, whereas only work harassment explained approximately a third of private sector nurses' turnover intentions. The findings suggest a differential impact of management on work harassment across the public and private sector.

Limitation; Cross-sectional data is susceptible to common method bias. A common latent factor was included, and several items that were explained by common method variance were controlled. Further, the findings are limited by the sample size from one sector and the use of only one developing country.

Implications: It is a waste of resources to transplant Anglo-American and European management models to developing countries without understanding the impact on nurses' outcomes.

Originality: Anglo-American and European management models and theories are not easily transferable to the Bangladesh context probably because of the impact of ties and corruption. 
Line management is a positive resource that can build employee wellbeing for public sector employees only.

Keywords: public-private sector, line management, well-being, work harassment, turnover intentions 


\section{Comparing the impact of line management on public and private nurses in Bangladesh}

Public sector management models do not easily translate to best practice in developing countries, mainly because the conditions are too different. For example, in contrast to developed countries, there are more doctors than nurses in Bangladesh and the demand for nurses' services exceeds supply whilst continuing to grow in line with population growth: hence they work in high-pressure environments (World Health Organization, 2018; World Bank, 2016). As a consequence, this paper examines whether Bangladeshi nurses perceive work harassment similarly to other public and private nurses in developed countries (Brunetto, Xerri, Trinchero, Farr-Wharton, Shacklock, Borgonovi, 2016) and whether this affects their turnover intention. Dick (2010) refers to work harassment as a form of workplace bullying but suggests that it differs from bullying. Specifically, an employee is exposed to work harassment when their employing organisation unreasonably monitors their actions, sets excessively high workloads, and has stringent accountability standards (Brunetto et al, 2016b). As such, the organisation and its managers are the instigators of work harassment.

Work harassment has been identified as a growing problem for public sector managers in developed countries (Tummers, Brunetto \& Teo 2016). For example, Brunetto et al. (2016b) compared work harassment of nurses in Australia and Italy and found significant parallels in their perceptions. More recent research identified a growing presence and acceptance of work harassment in US and UK hospitals (Farr-Wharton, Brunetto \& Xerri, 2019). The delivery of health services has been significantly affected by the cost-constraint that mandated the depth and breadth of reforms over the past four decades which has resulted in the expectation that employees will 'do more with less' (Diefenbach, 2009). However, the situation is even more pronounced in Bangladesh, where the shortages in nurses, especially in the public sector and in particular, in rural areas, is a growing problem (Ahmed \& Shirahada, 2017). Specifically, there are high levels of resource inadequacy, with widespread shortages in basic nursing 
equipment and a lack of an effective nurse management systems (World Health Organization, 2016). Such inadequacies in the Bangladesh system are likely to create a potential haven for work harassment.

The shortage of public sector nurses in Bangladesh is increasingly dire, and in some cases, the families of patients have to act as carers (Mahdy, 2009). If work harassment is not managed effectively, then it may negatively influence the profession's ability to attract new nurses, and retain them in the job. Whilst there is evidence of work harassment across different public sector occupations, such as local government and healthcare workers across different countries - Australia, Italy, USA (Tummers et al 2016; Xerri et al, 2016), there is no evidence as to whether work harassment is an issue in developing countries. In this study, we have two core aims. The first aim is to develop insight into the impact of line management on outcomes such as work harassment, wellbeing and turnover intentions for nurses working in Bangladeshi hospitals. The second aimis to develop a deeper understanding of the similarities and differences across the public and private sectors.

In relation to work harassment, in developed countries the theory argues that the role of the line manager for professionals is very important because of the dual role of the professional mentor and line manager within the organisational hierarchy. However, line managers of professionals are also increasingly faced with the issue of 'professional hybridity' (the sometimes-contradictory expectations that managers will both develop nurses' professional skills and knowledge and ensure task completion) (Noordegraaf, 2015). Never-the-less, a good line manager is one who can mediate the excessive demands of the hierarchy to ensure appropriate resourcing and support is available in relation to task completion and professional development (Farr-Wharton, Brunetto, \& Shacklock, 2011). Although past evidence has established the association between line management practices and wellbeing, and between line management practices and turnover intentions (Brunetto et al., 2013), to the best 
knowledge of the authors, there is no evidence about how work harassment may change these established relationships. As such, we aim to add to the existing evidence by examining the role of work harassment in affecting the association between satisfaction with line management and employee wellbeing, and in turn, turnover intentions within a developing country such as Bangladesh. Such evidence adds to the literature about the impact of line management on employee wellbeing and turnover intentions of nurses within developing countries, in this case, Bangladesh.

Managing both public and private sector employees in developing countries can be challenging as international HR systems are imported into existing culture-driven decisionmaking systems. Most managers were traditionally autocratic in their practices in South Asia (Khan, Miah \& Manzoor, 2014). However, in the last three decades, the service sector has undergone significant change mainly because of global forces and government reforms that have elevated the importance of human resources slowly, especially in terms of the significance of ensuring adequate qualifications and competencies for key positions. This change has led to the introduction of modern HRM and strategic systems, however, the new systems have not replaced the previous stronghold of culture in organisational decision-making. This means that family ties and corruption still dominate decision making, favouring some and disadvantaging the majority, and whilst those with strong international connections are likely to have basic HR policies and structures evident in countries such as the USA and UK, the difference is in their ability to implement those policies into practice (Khan et al., 2014, p 99). Hence, while Bangladeshi organisations may have specific policies about work hours and duties, in practice, there is probably greater scope for work harassment in Bangladesh compared with AngloAmerican countries such as the UK, USA and Australia.

This paper compares the relationship between line management, work harassment, wellbeing and turnover intentions of public and private sector nurses using a Conservation of 
Resources (COR) theoretical framework (Hobfoll, 1989). COR theory is typical of theories originally developed and tested within an Anglo-American and/or European context (history, culture) to explain employee outcomes regarding their ability to maintain and grow their personal resources such as health and wellbeing. However, it may not have as much relevance in explaining behaviour in developing countries. COR theorists argue that when the organisational environment erodes their personal resources through work harassment, their wellbeing is negatively impacted and this motivates them to protect their well-being by regulating their work practices. COR theorists argue that if extra support (e.g., from managers) is not available, then employees may offset perceived resource losses, and potential shortages, by reducing effort at work or leaving the organisation (Hobfoll, 2011). The research questions (RQs) guiding the study are:

$R Q$ 1: What is the impact of line management on work harassment, wellbeing and turnover of public and private sector Bangladeshi nurses?

RQ 2: What are similarities and differences in the relationship between line management, work harassment, wellbeing and turnover for nurses in the public and private sector?

The next part details a relevant review of the literature and identifies hypotheses that reflect the gap in the literature.

\section{Background}

\section{Conversation of Resources (COR) theory}

The COR theory is ideal for understanding employee outcomes, especially in the face of resource adversity (Hobfoll 1989). The way COR theory works is via two principles and four Corollaries (Hobfoll, 2011). Principle one: a loss of resources likely has a bigger effect than acquiring new resources. Principle two: to gain new or more resources, employees must firstly spend time or energy (resources) in those activities likely to lead to more resources. Further, if employees believe they are likely to experience a loss of resources (such as a loss 
of wellbeing) they will likely engage in activities (sick leave/ look for another job) to protect themselves against falling wellbeing. The four corollaries comprise four assumptions Firstly, the higher employees' perception of resources are, the easier it is for them to gain resources, whereas employees with fewer resources are more susceptible to resource loss, and less able to gain resources. Secondly, it is likely that an initial loss of resources will lead to further losses; whereas it is likely that a resource gain will likely lead to further resources gains. Fourthly,) employees are likely to act defensively as a strategy to prevent further loss of resources (Halbesleben, Neveu, Paustian-Underdahl, \& Westman, 2014).

Drawing from COR theory, each organisation makes decisions that produce either positive or negative environmental conditions, called 'resource caravan passageways' (Hobfoll (2011). Under ideal conditions, an employee can build resources such as increased wellbeing by investing other resources at their disposal; in this case, effective supervision is a social resource (caravan passageway) an employee can draw upon. Whereas, if the resource caravan passageway is high levels of work harassment, employees have low resources and are thus less able to orchestrate resource gain. In this case, we propose that work harassment may result in employees that are less able to develop wellbeing with the social resource derived from the relationship with their manager.

Using COR theory to explain the relationship between management, work harassment, wellbeing and turnover, we argue that effective management practices is a positive resource caravan passageway that can build wellbeing, whereas work harassment is a negative resource caravan passageway that will influence an employee's ability to develop resources such as wellbeing. The consequences of the poor environmental conditions are likely to lead to increased turnover (Hobfoll, 2001). Using COR corollary 4, the issue will be whether the support from the manager is enough of a 'cushion' to form a barrier against the negative influence of work harassment on wellbeing, and the flow on to an intention to turnover. As per 
corollary 4 , those with a lack of resources are likely to act defensively to conserve against further resource loss. That is, those who lack resources are unable to apply resources to fight against resource loss (Hobfoll, 2011), and thus to conserve further loss, they may choose to eject themselves from the situation by intending to leave their employing organisation. In contrast, those employees with adequate resources are likely to be able to invest them in resource development, which is likely to be evident by high wellbeing and low turnover intentions.

\section{Variables under examination}

\section{Work Harassment (resource caravan passageway)}

The term 'work harassment' is challenged by different groups of researchers and the same work has multiple meanings depending on the country. Originally, the term emerged from 'harassment' meaning “... all repeated behaviors [sic] which ... provoke, frighten, intimidate, or bring discomfort to the victim" (Brodsky (1976) cited in Einarsen, 2000; p382). However, the definition has moved on in different ways in different countries. For example, the Scandinavian researchers adopted different terms compared with the Anglo-American researchers for similar behavioural aggression and there remains no consensus about definitions for the different negative work behaviours (Branch, Ramsay, \& Barker, 2013; Einarsen, \& Nielsen, 2015). In terms of public sector HR in Anglo-American and/or European countries, work harassment has emerged as a negative work behaviour, similar to bullying or mobbing because it is characterised by repeated ill-treatment with negative consequences for the individual (Zapf, Escartin, \& Einarsen, 2011).

Originally, work harassment was conceptualised as part of bullying because of its negative impact on the individual (Raynor, 2002). More recently, work harassment is differentiated from bullying for three main reasons. Firstly, bullying is directed at an individual, whereas work harassment is about task completion and affects large numbers of front-line 
employees facing a chronic problem of demand outstripping supply, forcing them either to choose 'who gets what' (or who misses out) and/or 'to do more with less' on a continual basis (Dick, 2010; Xerri, Farr-Wharton, Brunetto, \& Lambries, 2016). Secondly, bullying can involve physical or mental abuse directed at an individual, whereas work harassment is not personal. Instead, it is the result of a resourcing decision made originally by governments, and then by senior management in allocating resources to each department (Tummers, et al, 2016).

Thirdly, the incidence of work harassment relies on the power of professional principles to ensure patients/clients/customers are serviced, irrespective of organisational resourcing constraints. This is because universities and the professional bodies regulate entry into the professions and work practices are underpinned by specific norms and values (such as professional standards of service and effectiveness) (Farr-Wharton et al., 2011). This in turn often brings them into conflict with organisational norms (task completion/ reduced per capita costs) leading to 'professional hybridity' (Noordegraaf, 2015). One of the outcomes for healthcare professionals is increasing stress from role conflict as professionals work overtime (often against organisational directions) (Tummers, Vermeeren, Steijn, \& Bekkers, 2012). Hence, work harassment is a type of negative workplace behaviour that is emerging as a growing HR problem, especially for healthcare professionals working in the public sector where austerity dominates resourcing decisions. In particular, Tummers et al. (2016) identified the rise in stress (and stress-related illnesses) emerging amongst professionals because of increasing work harassment, role overload and role ambiguity.

For healthcare workers, high demand is a worldwide phenomenon. In those countries where there is also a shortage of nurses, the workplace conditions are even more likely to enhance, rather than detract from work harassment. For example, Laschinger and Fida (2014) found that in Canada, even new graduate nurses were reporting high-stress levels leading to falling wellbeing and in the worst cases, there was a steady rise in the incidence of high levels 
of burnout and turnover. Laschinger et al. (2014, p19) argue that the key contributors are heavy workloads and inadequate staffing that in turn, create the negative environmental conditions ripe for increasing job dissatisfaction, and:

"Similar to other helping professions, the prevalence of burnout in nursing is particularly high, because of the high emotional and physical demands of this work"

A similar situation was identified for new nurses in the UK, where ethical leaders were identified as a resource countering negative work conditions (Mastracci, 2016). However, it may be the impact of cultural factors intervening for Bangladeshi nurses that negates the impact of work harassment if for example, they perceive the importance of helping pateints are overriding the impact of high workloads on their wellbeing. This is what is unknown - do Bangladeshi employees such as nurses behave similarly to employees in developed countries? In terms of COR theory, high work harassment acts as a negative work behaviour that erodes personal resources. If an employee has a shortage of resources - they will likely see a reduction in their wellbeing. As such, principle two is enacted and consequently, employees seek to control the negative flow of wellbeing by looking for another job (as one example of acting defensively) (Hobfoll 2011). Unless there is a counterbalance of positive resources, for example - positive relationships with their managers that can buffer and negate the impact of work harassment, then employees' personal resources will be negatively affected.

\section{Employee Resources (resource caravans)}

\section{Manager Support - Leader-member exchange (a social support resource)}

Manager-subordinate relationships can be conceptualised via the LeaderMember Exchange (LMX) framework. LMX is a Social exchange theory (SET) variable because the potential benefits from effective workplace relationships far outweigh the initial outlay of good will. LMX argues that managers form different quality relationships with 
employees: for some their workplace interactions are meaningful and over time leads to mutual reciprocity (insiders). On the other hand, for others the interactions are negative and consequently a relationship forms that is built only on the work contract specifications (Martin, Thomas, Guillaume, Lee, \& Epitropaki, 2016). The benefits of an effective LMX relationship were identified in a meta-analysis by Martin et al. (2016) as high role performance and high citizenship behaviour as well as low counteractive workplace behaviour. This relationship becomes even more important when it involves professionals because there is an added responsibility on the part of the manager to ensure the employee is socialised into the values, norms and practices associated with the profession and adheres to professional standards (Brunetto et al., 2018). However, research about public sector nurses in Brazil found that that the theories and "truths" accepted as the behaviour of employees such as nurses in developed countries does not always translate to the behaviour of nurses in BRIC countries. For example, Brunetto, Shacklock, Teo, Farr-Wharton \& Nelson (2015) found that managers had minimal impact on Brazilian nurses' behaviour for numerous reasons, including that there were different contracts in place with some nurses reporting to managers in the capital of Brazil, not the hospital, and the impact of corruption on nurses' actions in the workplace.

However, using COR theory, when LMX is high, it suggests that employees can access support from managers or someone in management. Hence, it is not surprising that previous research in developed countries suggests that high LMX is associated with high wellbeing and low turnover for nurses in Anglo-American and European countries such as Australia, Italy and the UK (Brunetto et al., 2018) as well as the USA (Brunetto et al., 2013). The reason as to why LMX is associated with high employee performance is because as Lashinger and Fida (2014) found in their research of Canadian nurses, high LMX indicates that nurses are receiving high levels of support from managers, which in turn provides a barrier to stress and helps maintain high employee wellbeing. However, the relationship has not been examined for public 
and private sector Bangladeshi nurses, where cultural influences on HR and workplace relationships do not necessarily follow the Anglo-American and/or European model. To test whether similar relationships are in play with Bangladesh for nurses, the following hypotheses are used.

\section{H1. LMX is negatively related to turnover intention}

H2. LMX is positively related to employee wellbeing

\section{Employee well-being (an individual/personal resource)}

'Well-being' is a general term with multiple definitions across different disciplines and there is no agreement on a definition except; however, that it is a multi-faceted concept (incorporating physical, job-related, psychological, social wellbeing), and each time it is used, it requires explicit defining in order to avoid confusion (Seligman, 2012). Employee well-being is defined as a composite state that encompasses a hedonic component (life satisfaction) and a eudaimonic component (living life according to one's values) (Forgeard, Jayawickreme, Kern, \& Seligman, 2011) within an occupational context (van der Doef and Maes 1999). Past research argues that high workloads erode nurses' well-being because wellbeing is the barometer indicating how well they are coping (Brunetto et al., 2017). Previous research shows that high wellbeing predicts low turnover for public sector Australian nurses (Brunetto, Rodwell, Shacklock, Farr-Wharton, \& Demir, 2016a) and high work harassment is associated with low wellbeing for Australian and Italian public and private sector nurses (Brunetto et al., 2016b).

\section{Turnover Intentions}

Turnover intentions refers to employees' propensity to withdraw from their present workplace and consequently, they conserve their energy at work and redirect it towards looking at alternatives (Van Knippenberg, Van Dick, \& And Tavares, 2007). There are multiple push factors likely to promote turnover intentions, including poor quality relationships and the lack of support given to staff (Van Knippenberg et al, 2007; Lashinger \& Fida, 2014) as well as low 
wellbeing for US and Australian nurses (Brunetto et al., 2013). In each case, high wellbeing was associated with low turnover intentions. The following hypotheses replicate this finding for Bangladeshi nurses.

H3. Employee wellbeing is negatively related to turnover intention

\section{Mediation and Moderations}

The way that work harassment and wellbeing impact the relationship between LMX and turnover for Bangladeshi public and private sectors is not clear. There are two types of possible effects. First, work harassment moderates the association between LMX and wellbeing, and between LMX and turnover intentions. If this is the case then it means that work harassment affects the direction or strength of the relationship between LMX and wellbeing, and LMX and turnover intentions (Baron \& Kenny, 1986). Second, wellbeing mediates the association between LMX and turnover intentions, which means that employee wellbeing accounts for some of the negative relationship between LMX and turnover intentions. The two types of effects will be discussed further in the following paragraphs.

In terms of COR theory, wellbeing is the measure of an individual's personal resources and is affected by both personal (such as psychological capital) and organisational (such as LMX) factors. In particular, high LMX is associated with high wellbeing for Australian public sector nurses (Brunetto et al., 2016). In addition, high wellbeing is associated with low turnover intentions for Australian and US nurses (Brunetto et al., 2013). This means that if employees have high wellbeing (perhaps because of high personal resources), it may mediate the relationship between LMX and turnover intentions. According to COR theory, if wellbeing is high enough, we expect that the personal resources gained from high wellbeing will mediate the impact of LMX on turnover. The following hypothesis expresses this premise.

H4. Employee wellbeing mediates the relationship between LMX and turnover intention 
Moderation refers to a situation where the relationship between two variables depends on a third variable. As stated, previous research shows that high LMX is associated with high wellbeing and high work harassment is associated with low wellbeing. In the context of Bangladeshi nurses, we propose that high work harassment results in employees feeling overworked, under-resourced, and that overall their employing organisation's expectations are unrealistic. In such a work environment, even if employees have a good relationship with their manager, it is expected that high work harassment will reduce the positive impact of LMX and employee wellbeing, and decrease the negative impact of LMX on turnover intention. Moreover, we expect that work harassment will moderate the impact of LMX because a manager is considered an agent of the organisation (Eisenhardt, 1989), and thus unrealistic expectations may flow through the manager to the nurses. Therefore, if a nurse thinks expectations are unrealistic, they may associate such expectations with the manager, which when work harassment is high will reduce the positive influence of the manager on employee wellbeing and reduce the negative affect on turnover intentions.

\section{H5. Work harassment moderates the relationship between LMX and wellbeing}

\section{H6. Work harassment moderates the relations between LMX and turnover intentions}

\section{The public-private context}

The context of this research is a comparison of the public and private sector work environment for Bangladeshi nurses. The public sector in Bangladesh is highly bureaucratic and hierarchical, and is described as being over-governed (micro-managed and autocratic) with insufficient or inappropriate governance (subject to corruption) (Ahmed \& Shirahada, 2017). This means that there is a lack of separation between the official practices of public sector officials and personal interests, and a growing realisation that recent reforms, whilst in theory about re-distributing resources from the rich to the poor, did not achieve the stated goals (Khan 
et al, 2014). Also despite specific legislation promoting equal rights for women, reforms have not made much of an indentation on the glass ceiling imposed upon women by the dominant culture in play (Jamil, Aminuzzaman \& Hoque, 2013). According to Ahmad et al. (2007) (cited in Ghuman \& Singh, 2013) reforms were supposed to provide greater access to healthcare for the poor. However, in practice, the rich were given greater access to resources, and nurses were dissuaded against going to poorer regions because of inadequate infrastructure facilities. In summary, reforms did not achieve a greater focus on efficiency or achieving outcomes. Hence, LMX, work harassment, wellbeing and turnover is expected to be worse for public sector nurses. In the other hand, Farr-Wharton et al (2019) found work harassment was also prevalent for UK and US private sector nurses. The hypothesis is:

H7: Private sector employees perceive higher LMX and wellbeing and lower work harassment and turnover compared with public sector nurses.

\section{Method}

Sample and procedure

In the first instance, nurses who had completed an undergraduate and/or postgraduate nursing qualification from the Grameen Caledonian College of Nursing, Dacca, Bangladesh were invited to complete the survey. In total, 191 useable surveys were returned (redemption rate of $62 \%$ ). Additionally, 126 useable surveys were received from nurses working in two public sector hospitals in Dacca, Bangladesh. The data was collected in 2017 and 2018.

In total, 317 useable surveys were obtained, 131 from the public sector and 186 from the private sector. The demographic data depicts that unlike the ageing nursing workforce in many OECD countries (World Health Organization, 2018); the majority of nurses sampled in the Bangladesh public and private sectors were less than 31 years of age. As well, many have less than one-year employment with their current hospital, with the majority having less than 
5 years employment in their current hospital. In the public sector, there were 34.4 per cent males $(n=45)$ and 65.6 per cent females $(n=86)$, which is a stark comparison to the private sector with 4.3 per cent males $(n=8)$ and 95.6 per cent females $(n=178)$.

\section{Measures}

All items for each scale were scored on a six-point Likert scale from 1 (strongly disagree) to 6 (strongly agree). The reliability for each scale is depicted in Table 1 .

Leader-member exchange. To measure a nurse's relationship with their line manager, we used the Leader-Member Exchange (LMX) instrument developed by Graen and Uhl-Bien (1995). An example of an item is 'My manager is satisfied with my work.'

Employee wellbeing. We measured an employee's sense of their wellbeing with the four items from Brunetto, Farr-Wharton, and Shacklock (2011). A sample item includes 'Overall, I am reasonably happy with my work life.'

Turnover intentions. To identify nurse intentions to leave their employing organisation, we used three-items adopted from Meyer, Allen, and Smith (1993) scale of turnover intentions, including, 'I frequently think about leaving my current employer.'

Work harassment. Rayner and Cooper (2006) developed a scale to measure task-attack, personal-attack and intimidation. To measure work harassment, we used three items that examine 'task-directed attack,' including the item '[This hospital] sets unrealistic targets.'

Control variables. Many variables within the nursing and turnover intention literature may impact turnover intentions. We controlled for tenure within a hospital because research has found those with longer tenure have a lower intention to leave their employing organisation (Ng \& Feldman, 2009b). Although there is limited agreement in the literature, we also controlled for gender, with one study suggesting women quit their job less than their male counterparts (e.g., Lyness \& Judiesch, 2001), and a larger scale study of 475,000 professionals and managers concluding women were more likely to voluntarily leave. Finally, we controlled 
for age because of $\mathrm{Ng}$ and Feldman's (2009a) postulation that older employees typically develop stronger ties to their colleagues and their employing organisations.

\section{Data analysis}

To test a moderated mediation model, we used SPSS v.25 and the PROCESS Macro by Andrew Hayes (Hayes, 2018). The PROCESS macro is a better alternative to test mediation when compared to approaches such as that prescribed by Baron and Kenny (1986), as there is no requirement for the data to be normally distributed. Within the PROCESS macro, we used model 8 and applied 95 per cent bootstrap confidence intervals across a sample of 5000. To confirm the factor structure and to account for common method variance, we undertook a confirmatory factor analysis of the public and private sectors. To check and control for common method variance, we included a common latent factor (Podsakoff, Mackenzie, Lee, \& Podsakoff, 2003).

\section{Results}

\section{Descriptive statistics and intercorrelations}

Table 1 depicts the descriptive statistics, scale reliability and intercorrelations. Notably, LMX did not significantly influence turnover intentions in the public sector. Although not significant, the $\mathrm{p}$-value was nearing statistical significance $(\mathrm{p}=0.06)$.

\section{Confirmatory factor analysis}

The hypothesised measurement model resulted in a poor fitting model for the private $(\mathrm{CMIN} / \mathrm{DF}=2.330, \mathrm{RMSEA}=0.085, \mathrm{CFI}=0.886$ and $\mathrm{SRMR}=0.066)$ and public $(\mathrm{CMIN} / \mathrm{DF}$ $=3.222, \mathrm{RMSEA}=0.131, \mathrm{CFI}=0.857$ and SRMR $=0.081)$ sectors. Due to factor loadings below 0.5 , a number of observed items were removed. We removed three LMX questions: 1. 'Regardless of how much formal authority he/she has built into his/her position, what are the chances that your leader would use his/her power to help you solve problems 
in your work? (What are the changes that you would)'; 2. 'have enough confidence in my leader that I would defend and justify his/her decision if he/she were not present to do so?'; and 3. 'How would you characterize your working relationship with your leader?'. We also removed one wellbeing item, 'I get enough time to reflect on what I do in the workplace'. The modified measurement model fit the data well for the private $(\mathrm{CMIN} / \mathrm{DF}=1.89, \mathrm{RMSEA}=.069, \mathrm{CFI}=$ .945 and $\mathrm{SRMR}=.058)$ and public $(\mathrm{CMIN} / \mathrm{DF}=1.98, \mathrm{RMSEA}=.087, \mathrm{CFI}=.953$ and $\mathrm{SRMR}$ $=.067)$ sectors. We tested a number of alternate models to establish the distinctiveness of the variables and the modified measurement model. As depicted in Table 2, the fit of the alternate models was significantly worse when compared with the modified measurement model for the private and public sectors.

The addition of a common latent factor (CLF) to the modified measurement model (model 4) for the private and public sectors resulted in improved model fit. In addition, a chisquare difference test for each sample revealed that the CLF model was statically different from the modified measurement model, and thus common method variance may explain a significant portion of the variance. To identify where common method variance was problematic, we compared the standardised factor loadings of the modified measurement model and the CLF model. To the best knowledge of the authors, there are no published cut-off values for the comparison of standardised factor loadings. We considered any loading difference above 0.20 to be excessively large and retained the CLF control. The final measurement model for the private and public sector include the bias-corrected estimates (i.e., controlling for common method bias). Specifically, to be able to test the hypotheses within the PROCESS macro, we imputed the bias-correct composite variables from AMOS into SPSS.

Testing the hypotheses

Figure 1 depicts the results for hypotheses 1-3 for the public and private sector. LMX predicts turnover $(\mathrm{H} 1)$ for private sector employees only and LMX predicts wellbeing $(\mathrm{H} 2)$ for 
both public and private sector and wellbeing predicts turnover intentions (H3) for public sector employees only. There was support for the acceptance of hypothesis 4 (mediation), with a significant indirect effect $\mathrm{SE}=-.21, \mathrm{CI}(-.42,-.10)$. As mentioned, the direct effect between LMX and turnover intentions was not significant, providing support that wellbeing completely mediates the relationship between LMX and nurse turnover intentions in the Bangladesh public sector. We also found work harassment did not moderate the relationship between LMX and turnover intentions (i.e., rejecting hypothesis 6), but, as depicted in Figure 2, work harassment did moderate the path between LMX and employee wellbeing (i.e., providing support for the acceptance of hypothesis 5). Specifically, high work harassment reduced the influence of LMX on employee wellbeing.

In relation to the private sector, wellbeing did not directly relate to turnover intentions, so we rejected hypothesis 2 . Due to the rejection of hypothesis 3 , and insignificant indirect effects, we rejected hypothesis 4 . Finally, work harassment did not moderate the relationship between LMX and wellbeing, or LMX and turnover intentions. As such, we rejected hypotheses 5 and 6 .

\section{Discussion}

This paper examines the impact of line management on work harassment, wellbeing and turnover of public and private nurses in Bangladesh using a COR framework. One factor which has been identified as having a negative impact, especially in the public sector, is work harassment and in developed countries, the manager either alleviates the situation or makes it worse (Brunetto et al, 2016b). In contrast, Farr-Wharton et al (2019) also identified work harassment for private sector nurses in the UK and USA. However, research in Brazil found that management did not have the same impact compared with Anglo-American and/or European countries such as UK and Australia (Brunetto et al, 2015), hence, we examined the impact of management on public and private nurses' perceptions of work harassment in 
Bangladesh. Additionally, since, private sector nurses operate in hospitals that have attempted to implement some typically western HR systems, it seems likely that turnover should be less because the influence of culture (corruption etc) should be less.

In terms of the first and second hypotheses, previous research had already shown that effective management is associated with high wellbeing and low turnover intentions for public and private sector employees in Anglo-American and European countries (Martin et al., 2016; Brunetto et al., 2018, 2013). The findings from this research support previous research showing that high LMX is associated with high wellbeing for both public and private sector nurses in Bangladesh. The role of the line manager is to act as a source of support when workloads are physically and mentally draining - hence in the ideal situation, they provide a barrier to stress, in turn supporting high wellbeing (Lashinger \& Fida, 2014). However, high LMX is only associated with low turnover for private sector nurses in Bangladesh. One explanation is that public sector nurses are unlikely to have a modern HR system in place (of which a basic manager-employee relationship is an important cornerstone), and instead, the traditional culture may be more important in determining whether employees stay or leave (Ahmed \& Shirahada, 2017; Khan et al., 2014). Khan et al (2014) argues that in Bangladesh, the policy may be similar to a developed country, but the implementation of HR policies has been challenging, especially in the public sector. Hence, it seems likely that family ties and corruption still dominate decision making, in turn negating the role of managers.

Additions, the findings suggest that in contrast to the role of line managers of professionals in Anglo-American and European countries, who play two distinct (sometimescontradictory) roles - professional hybridity, firstly developing nurses' professional skills and knowledge, while still acting as their line manager (Noordegraaf, 2015); the role of public sector line managers in Bangladesh is unclear because nurses' wellbeing is determined by how work harassment mediates the relationship between LMX and wellbeing. One possible reason 
is that their managers are not engaging in professional activities such as socialising them into the professional norms and values associated with nursing. Khan et al (2014) suggests that in countries like Bangladesh, increased professionalization of the Bangladeshi public sector has failed to change 'who gets what' and how workplaces are organised (Jamil, 2013). Hence LMX may not be based on professional factors and the managers may not act as positive antecedents of nurses' wellbeing as is the case in developed countries (Martin et al, 2016). However, further research should examine why a significant relationship was absent.

Regarding hypothesis 3, previous research had already shown that high wellbeing was associated with low turnover intentions for nurses in the USA and Australia (Brunetto et al., 2016). In terms of COR theory, high wellbeing is an important personal resource that employees want to develop, and conserve when required (Hobfoll 2011). The findings from this research are consistent with past research and COR theory for public sector Bangladeshi nurses only. More research is required to investigate the relationship for private sector nurses. It may be that the traditional culture has a role to play in better understanding the relationship between wellbeing and turnover for Bangladeshi nurses working in the private sector (Khan et al., 2014). Such insight may better explain how employees build wellbeing in the workplace, and importantly, why they stay even in workplace enrivonments that deplete their wellbeing.

The mediation and moderation processes shed further light on understanding the differences in the public and private workplaces for nurses in Bangladesh. Past research shows that work harassment is a product of the era of austerity, which now governs the work lives of many professionals in the caring industry, especially in the public sector in developed countries. However, very little is known about how reforms (if any?) have affected the professional workplace in developing countries. In ideal circumstances, professionals expect their line managers to mediate unrealistic organisational work demands and provide appropriate and sometimes-extra support resources to ensure both task completion and professional 
development occurs (Farr-Wharton et al., 2011). In this study, work harassment does mediate the relationship between LMX and wellbeing for public sector nurses, but not for private sector nurses. Over half of public sector Bangladeshi nurses' wellbeing is explained by how work harassment mediates the relationship between LMX and wellbeing.

While previous research shows that LMX predicts work harassment (Farr-Wharton et al., 2018), and work harassment predicts employees' wellbeing (Xerri et al., 2016), this paper adds new evidence about the role of work harassment in moderating the relationship between management and wellbeing of public sector nurses in Bangladesh. More specifically, in the public sector, when work harassment was low, management had a greater positive influence on employee wellbeing. These results provide new evidence about the influence of work harassment on the well-established association between management practices and wellbeing. This means that the role of the manager is just as important in developing countries such as Bangladesh as it is in developed countries, although culture may affect how it works. It also aligns with COR theory in that those with fewer resources are less able to apply resources to develop other resources, which suggests that those managers who support their nurses impact their decision to stay by improving their wellbeing. However, this is the case for public sector nurses only.

In contrast, LMX explains just under a quarter of private sector nurse wellbeing, and work harassment has no significant impact on this association. Further, there is no evidence of work harassment moderating the relationship between LMX and turnover in this study, although, high LMX, work harassment and wellbeing does explain just under a third of the turnover intentions of public sector Bangladeshi nurses. In the case of private sector Bangladeshi nurses, work harassment alone explains well over a third of their turnover intentions. This contrasts research by Farr-Wharton et al (2019) about US and UK private sector nurses and suggests that the manager in Bangladesh may not have the power to affect 
how work harassment influences turnover intentions for private sector nurses. Hence these research findings differ from those in the Anglo-American and/or European countries which may suggest a significantly different work context for these private sector nurses working in one of the least developed countries in the world. The findings provide a platform for more research about management issues facing public and private professionals such as nurses in developing countries such as Bangladesh.

The last hypothesis examined the differences between the public and private sector nurses' level of satisfaction with LMX, work harassment, wellbeing and turnover. The belief was that private sector nurses would perceive higher LMX and wellbeing and lower work harassment and turnover intentions compared to their private sector counterparts. The hypothesis was based on information that public sector reforms have not been successfully implemented, the public sector in Bangladesh is poorly resourced, and nurses are actively dissuaded from going to poorer regions because of inadequate infrastructure facilities (Jamil, 2013). However, the hypothesis was rejected because, across every factor, public sector nurses were more satisfied with management, had higher wellbeing, perceived lower work harassment and turnover intentions (See Table 1). One explanation using COR theory is that private sector nurses' wellbeing is being conserved and developed from other resources not examined in this paper. Future studies should further examine the antecedents, mediators and moderators relating to work harassment.

In reviewing this study, there are some limitations to consider. First, the cross-sectional data was susceptible to common method bias (Podsakoff et al., 2003). However, as mentioned, a common latent factor was included, and several items that were explained by common method variance were controlled, as per George and Pandey's recommendations (2017). Nonetheless, future research should examine work harassment over time to improve insight and design relating to common method bias. Second, although a study on Bangladeshi nurses 
provides some insight into work harassment in developing countries, further research is required about other developing countries. Further, another limitation relates to the sample in that most of the nurses had worked less than five years in their current employment, and therefore, there may be other factors, other than LMX and wellbeing affecting their intention to stay that have not been examined in this paper. Finally, the findings are limited by the sample size for each sector, and the type of demographic information collected that could have assisted in explaining it. Further research should be undertaken to increase the sample size, which will improve the stability of the results, and provide enough statistical power to examine more complex research models, including a thourough examination of work harassment antecedents, mediators and moderators.

\section{Conclusion}

The findings suggest that work harassment is prevalent in the Bangladesh public and private nursing profession. This study used a COR theoretical framework to compare the impact of management practices (LMX) and work harassment on the wellbeing and turnover intentions of Bangladeshi public and private nurses. Using COR theory, we found that LMX is a positive resource caravan passageway that can build wellbeing for public sector employees; however, the association was not significant for private sector nurses. Hence, COR theory principle 2 offers one explanation of $r$ public sector employees' behaviour in that it assumes that the manager provided enough of a 'cushion' for them to form a barrier against the negative influence of work harassment on their wellbeing (Hobfoll, 2001). Similar to what past AngloAmerican and European research has shown, the consequences of poor environmental conditions, such as work harassment, is that it does affect how public sector nurses in Bangladesh are able to take advantage of the relationship with their manager. However, there was no evidence that employees who perceive high support from their line managers are less 
likely to be negatively influenced by work harassment (Hobfoll, 2011). Therefore, whilst COR theory was somewhat useful in explaining some behaviour of public sector Bangladeshi nurses, it was far less relevant for explaining the behaviour of those in the private sector. This is an important finding because theories and concepts developed and validated in Anglo-American contexts assume underlying cultural similarities that may not be accurate in developing countries. Future research should perhaps test other well established theories (and concepts) across multiple contexts to verify their validity.

The implication of this research relates directly to public sector management. For example, the impact of management is different in Bangladesh compared with past AngloAmerican and European studies, and one factor is likely to be the traditional cultural stronghold, especially the impact of ties and corruption, which affects nurses' turnover intention, especially in public sector nurses areas (Ahmed \& Shirahada, 2017). Future research should explore those assertions. Additionally, there are significant differences in how public and private sector nurses respond to, and cope with work harassment. Hence, simply transplanting management models from developed countries for managing healthcare in developing countries may not yield its intended benefits. More research is required to better understand how managers affect how professionals work in developing countries. 


\section{References}

Ahmed, T \& Shirahada, K (2017) Toward a sustainable healthcare service system in a limited resource context: Case study of Bangladesh rural advancement committee's healthcare system, International Journal of Healthcare Management,

DOI: $10.1080 / 20479700.2017 .1389512$

Baron, R. M., \& Kenny, D. A. (1986). The moderator-mediator variable distinction in social psychological research: Conceptual, strategic, and statistical considerations. Journal of Personality and Social Psychology, 51(6), 1173-1182.

Branch, S., Ramsay, S., \& Barker, M. (2013). Workplace bullying, mobbing and general harassment: A review. International Journal of Management Reviews, 15, 280-299

Brunetto, Y., Farr-Wharton, R., \& Shacklock, K. (2011). Using the Harvard HRM model to conceptualise the impact of changes to supervision upon HRM outcomes for different types of public sector employees International Journal of Human Resource Management 22(3), 553-573.

Brunetto, Y., Rodwell, J., Shacklock, K., Farr-Wharton, R., \& Demir, D. (2016a). The impact of individual and organizational resources on nurse outcomes and intent to quit. Journal of advanced nursing, 72(12), 3093-3103.

Brunetto, Y., Xerri, M., Shriberg, A., Farr-Wharton, R., Shacklock, K., Newman, S., \& Dienger, J. (2013). The impact of workplace relationships upon engagement, wellbeing, commitment and turnover for nurses in Australia and USA. Journal of advanced nursing, 69(12), 27862799.

Brunetto, Y., Xerri, M., Trinchero, E., Beattie, R., Shacklock, K., Farr-Wharton, R., \& Borgonovi, E. (2018). Comparing the impact of management on public and private sector nurses in the UK, Italy, and Australia. Public Management Review, 20(4), 525-544.

Brunetto, Y., Xerri, M., Trinchero, E., Farr-Wharton, R., Shacklock, K., \& Borgonovi, E. (2016b). Public-Private Sector Comparisons Of Nurses' Work Harassment Using Set: Italy and Australia. Public Management Review, 18(10), 1479-1503.

Brunetto, Y., Shacklock, K., Teo, S., Farr-Wharton, R., \& Nelson, S. (2015). Nurses' managers, learning options and organisational commitment: Australia, Brazil and England. Journal of Nursing Management. 23 (8), 1029-1038

Dick, G. P. (2010). Can the organisation and supervision environment influence both bullying and organisational commitment? Evidence from a police force survey. Kent University Working Paper Series 196.

Diefenbach, T. (2009). New public management in public sector organizations: the dark sides of managerialistic 'enlightenment'. Public Administration, 87(4), 892-909.

Einarsen, S. (2000). Harassment and bullying at work: A review of the scandinavian approach. Aggression and Violent Behavior, 5(4): 379-401

Einarsen, S., Hoel, H., \& D. Zapf, D. eds. (2011). Bullying and Harassment in the Workplace: Developments in Theory, Research and Practice (2 ${ }^{\text {nd }}$ Edition). CRC Press, Taylor \& Francis Group, Boca Raton, Florida.

Einarsen, S., \& Nielsen, M. (2015). Workplace bullying as an antecedent of mental health problems: A five-year prospective and representative study. International Archives of Occupational and Environmental Health. 88: 131-142

Eisenhardt, K. M. (1989). Agency theory: An assessment and review. Academy of Management Review, 14(1), 57-74.

Farr-Wharton, R., Brunetto, Y., \& Shacklock, K. (2011). Professionals' manager-subordinate relationships, autonomy and commitment in Australia: a leader-member exchange theory perspective. The International Journal of Human Resource Management, 22(17), 3496-3512.

Forgeard, M. J., Jayawickreme, E., Kern, M. L., \& Seligman, M. E. (2011). Doing the right thing: Measuring wellbeing for public policy. International journal of wellbeing, 1(1), 79-106. 
George, B., \& Pandey, S. (2017). We know the Yin, but where is the Yang? Toward a balanced approach on common source bias in public administration scholarship. Review of Public Personnel Administration, 37(2), 245-270

Ghuman, B. S., \& Singh, R. (2013). Decentralization and delivery of public services in Asia. Policy and Society, 32, 7-21

Graen, G., \& Uhl-Bien, M. (1995). Relationship-based approach to leadership: Development of leader-member exchange (LMX) theory of theory over 25 years: Applying a multi-level multidomain perspective. Leadership Quarterly, 6(2), 219-247.

Halbesleben, J. R., Neveu, J.-P., Paustian-Underdahl, S. C., \& Westman, M. (2014). Getting to the "COR" understanding the role of resources in conservation of resources theory. Journal of management, 40(5), 1334-1364.

Hayes, A. F. (2018). Introduction to mediation, moderation, and conditional process analysis: $A$ regression-based approach. New York: Guilford Press.

Hobfoll, S. E. (1989). Conservation of resources: A new attempt at conceptualizing stress. American psychologist, 44(3), 513-524.

Hobfoll, S. E. (2001). The influence of culture, community, and the nested-self in the stress process: advancing conservation of resources theory. Applied psychology, 50(3), 337-421.

Hobfoll, S. E. (2011). Conservation of resource caravans and engaged settings. Journal of occupational and organizational psychology, 84(1), 116-122.

Jamil, I., Aminuzzaman, S., \& Haque, S. (2013) Introduction: Governance in South, Southeast, and East Asia Public Organization Review 13 (4) 341- 347

Khan., R, Miah., M, \& Manzoor., A (2014). Human Resource Management Practices: A Case Study of South Asian Countries IBT Journal of Business Studies 9(1), 83-101

Lyness, K. S., \& Judiesch, M. K. (2001). Are female managers quitters? The relationships of gender, promotions, and family leaves of absence to voluntary turnover. Journal of applied Psychology, 86(6), 1167-1178.

Mahdy, H. A. (2009) Reforming the Bangladesh healthcare system, International Journal of Health Care Quality Assurance, 22(4), 411-416.

Martin, R., Thomas, G., Guillaume, Y., Lee, A. \& Epitropaki, O. (2016). Leader-member Exchange (LMX) and Performance: A Meta-analytic Review. Personnel Psychology, 69, 67-121.

Meyer, J. P., Allen, N., \& Smith, C. A. (1993). Commitment to organizations and occupations: extension and test of a three-component conceptualization. Journal of applied Psychology, 78(4), 538-551.

Mastracci, S. (2016). Beginning nurses' perceptions of ethical leadership in the shadow of Mid Staffs. Public Integrity, 19(3), 250-264.

Ng, T. W., \& Feldman, D. C. (2009a). Age, work experience, and the psychological contract. Journal of Organizational Behavior, 30(8), 1053-1075.

$\mathrm{Ng}, \mathrm{T}$. W., \& Feldman, D. C. (2009b). Re-examining the relationship between age and voluntary turnover. Journal of Vocational Behavior, 74(3), 283-294.

Noordegraaf, M. (2015) Hybrid Professionalism and Beyond: (New) Forms of Public Professionalism in Changing Organizational and Societal Contexts, Journal of Professions and Organization, 2, 187-206.

Podsakoff, P. M., MacKenzie, S. B., Lee, J.-Y., \& Podsakoff, N. P. (2003). Common method biases in behavioral research: a critical review of the literature and recommended remedies. Journal of applied Psychology, 88(5), 879-903.

Rayner, C., \& Cooper, C. L. (2006). Workplace bullying. In K. E. Kelloway, J. Barling, \& J. J. H. Jr. (Eds.), Handbook of workplace violence. Thousand Oaks: Sage Publications.

Laschinger, H.K.S., Fida, R., (2014). New nurses' burnout and workplace wellbeing: the influence of authentic leadership and psychological capital. Burnout Research 1 (1), 19-28

Seligman, M.E., (2012). Flourish: A visionary new understanding of happiness and well-being. New York: Simon and Schuster. 
Tummers, L., Vermeeren, B., Steijn, B., \& Bekkers, V. (2012). Public professionals and policy implementation: Conceptualizing and measuring three types of role conflicts. Public Management Review, 14(8), 1041-1059.

Tummers, L., Brunetto, Y., Teo, S., (2016) Workplace aggression: Introduction to the special issue and future research directions for scholars, International Journal of Public Sector Management, 29 (1), 2 - 10

Van Knippenberg, D., Van Dick, R., \& and Tavares, S. (2007). Social Identity and Social Exchange: Identification, Support, and Withdrawal from the Job. Journal of Applied Social Psychology, 37(3), 457-477.

Van der Doef, M. and Maes, S., 1999. The job demand-control (-support) model and psychological well-being: a review of 20 years of empirical research. Work \& stress, 13(2), pp.87-114.

World Bank. (2016). Overview of Bangladesh. Retrieved from http://www.worldbank.org/en/country/bangladesh/overview

World Health Organization. (2016). World Health Statistics 2016: Monitoring Health for the Sustainable Development Goals (SDGs). World Health Organization. Retrieved from http://www.who.int/gho/publications/world health statistics/2016/en/

World Health Organization. (2018). Nursing and Midwifery Fact Sheet February 2018. Retrieved from http://www.who.int/mediacentre/factsheets/nursing-midwifery/en/

Xerri, M., Farr-Wharton, R., Brunetto, Y., \& Lambries, D. (2016). Work harassment and local government employees: Australia and USA. International Journal of Public Sector Management, 29(1), 54-71.

Zapf, D., Escartin,J., Einarsen, S., Hoel, H. \& Vartia, M. (2011). Empirical findings on the prevalence and risk groups of bullying in the workplace. In Bullying in the Workplace; Developments in Theory, Research and Practice edited by Einarsen, S., Hoel, H., Zapf, D., Cooper. C. CRC press, Taylor \& Francis group, Boca Rata 
Table 1. Correlation matrix

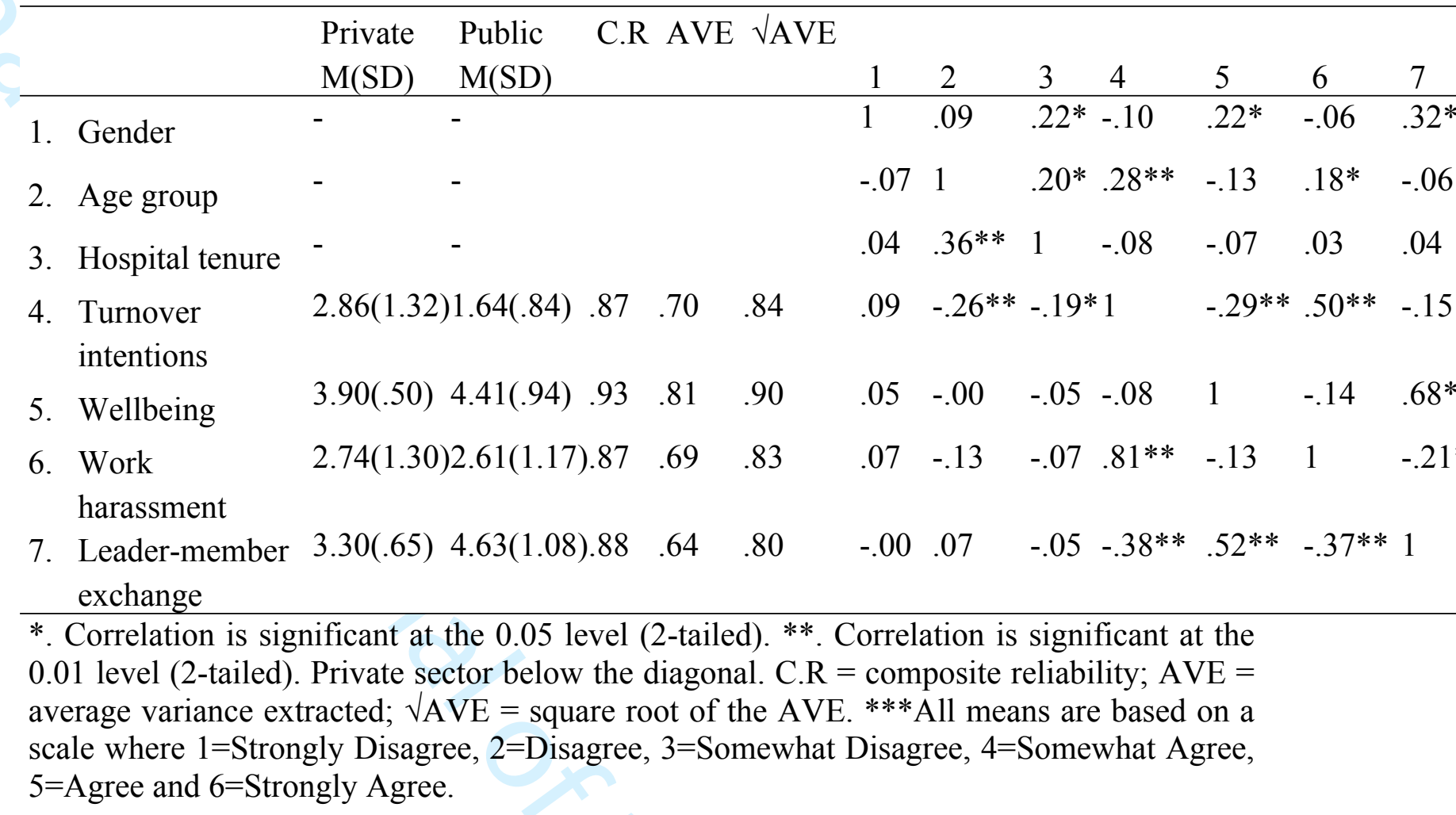

Table 2. Comparison of alternate measurement models

\begin{tabular}{|c|c|c|c|c|c|c|c|}
\hline Model & & $\chi^{2}$ & $\Delta \chi^{2}$ & df & $\Delta \mathbf{d f}(p)$ & CFI & SRMR \\
\hline & $\begin{array}{l}\text { Hypothesised measurement model } \\
\text { Private sector }\end{array}$ & 265.217 & & 113 & & .886 & .066 \\
\hline Base & $\begin{array}{l}\text { Modified measurement model - private } \\
\text { sector }\end{array}$ & 111.608 & & 59 & & .945 & .058 \\
\hline 1 & Combine LMX and wellbeing & 181.715 & 70.11 & 62 & $3(\mathrm{p}<.001)$ & .874 & .084 \\
\hline 2 & Combine LMX and Harassment & 317.966 & 206.36 & 62 & $3(\mathrm{p}<.001)$ & .731 & .146 \\
\hline 3 & Combine wellbeing and harassment & 229.781 & 118.7 & 62 & $3(\mathrm{p}<.001)$ & .824 & .118 \\
\hline \multirow[t]{2}{*}{4} & Add CLF to Base model & 76.394 & 35.21 & 46 & $13(\mathrm{p}<.001)$ & .968 & .045 \\
\hline & $\begin{array}{l}\text { Hypothesised measurement model (four } \\
\text { factor model) - Public sector }\end{array}$ & 364.098 & & 113 & & .857 & .081 \\
\hline Base & $\begin{array}{l}\text { Modified measurement model - public } \\
\text { sector }\end{array}$ & 116.799 & & 59 & & .953 & .067 \\
\hline 1 & Combine LMX and wellbeing & 309.179 & 192.38 & 62 & 3( & .797 & .090 \\
\hline 2 & Combine LMX and Harassment & 326.026 & 209.23 & 62 & $3(\mathrm{p}<.001)$ & .783 & .161 \\
\hline 3 & Combine wellbeing and harassment & 332.380 & 215.58 & 62 & $3(\mathrm{p}<.001)$ & .778 & .164 \\
\hline 4 & Add CLF to Base model & 69.596 & 42.203 & 46 & $13(\mathrm{p}<.001)$ & .981 & .046 \\
\hline
\end{tabular}




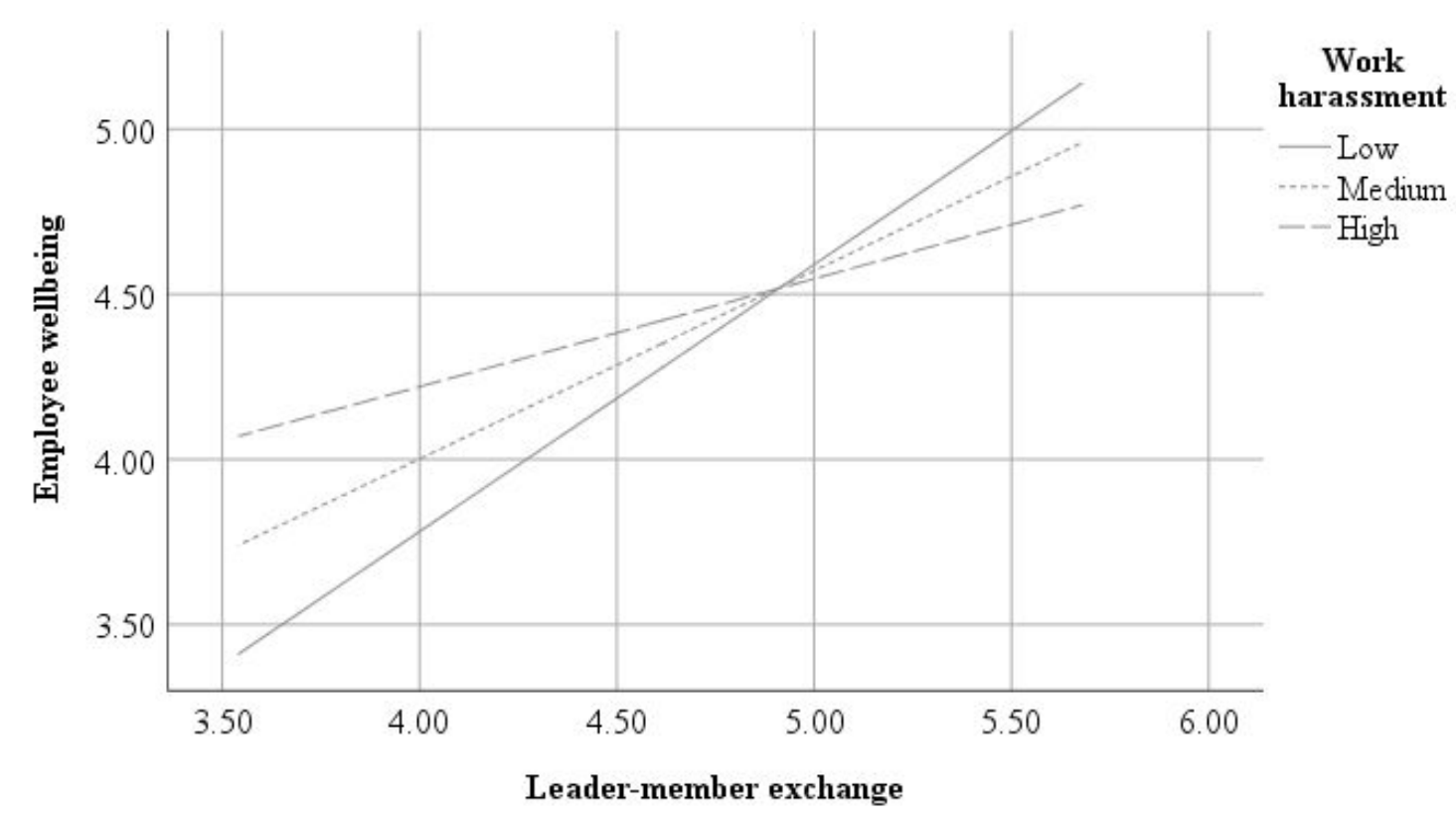

Figure 2. Moderation results - work harassment moderates the relationship between LMX and employee wellbeing. Note: Work harassment standardised for moderation analysis 


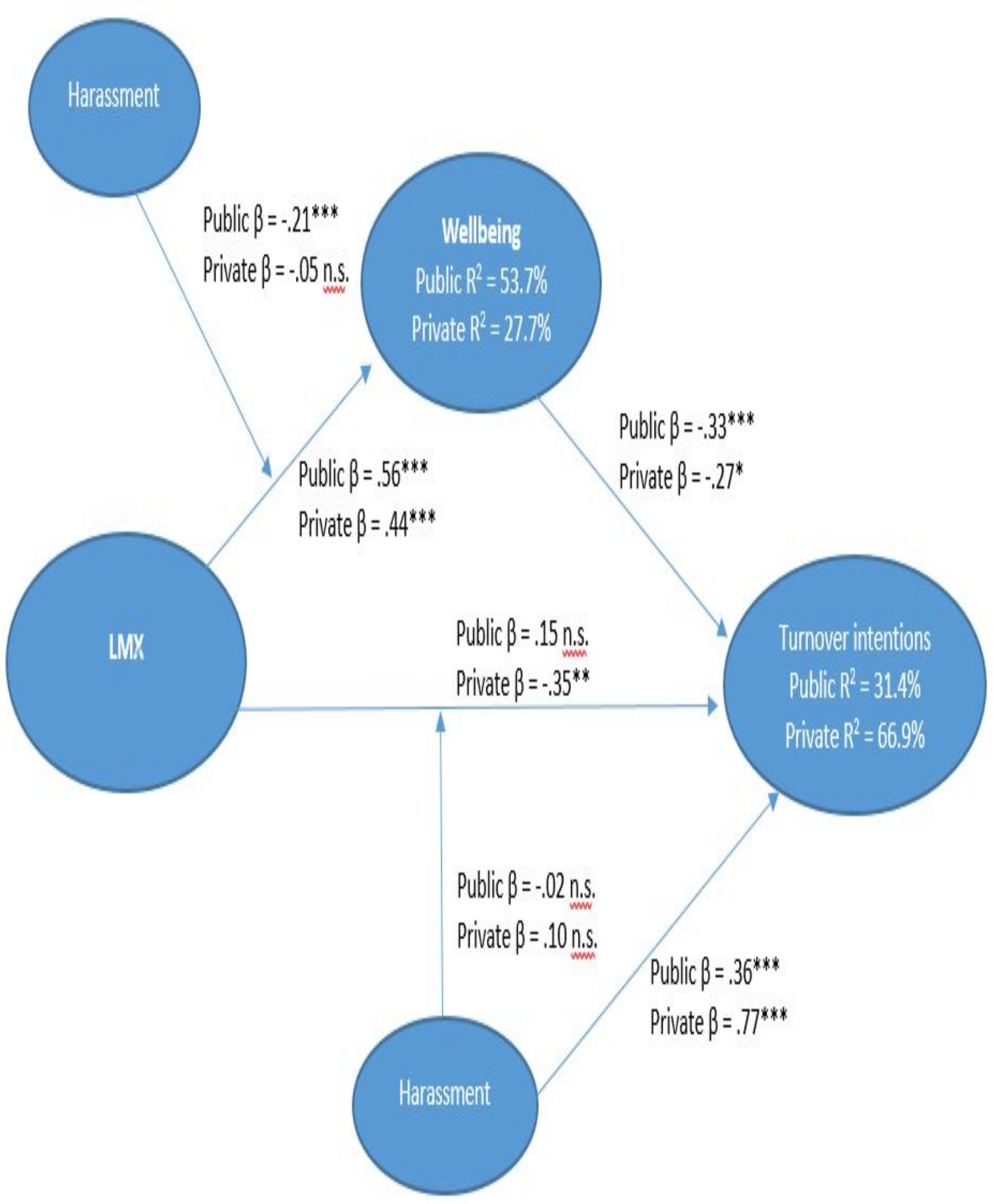




\section{A review of Manuscript ID IJPSM-01-2019-0023.R1 entitled "Comparing the impact of management on public and private nurses in Bangladesh"}

We thank the reviewers for their insightful comments and have responded accordingly. The changes made are detailed in the following document.

\begin{tabular}{|c|c|}
\hline Reviewers comments & Authors responses \\
\hline $\begin{array}{l}\text { In the Abstract, where "Findings" are } \\
\text { described, the authors should clarify the } \\
\text { differences between the public and private } \\
\text { sectors. As currently described, work } \\
\text { harassment is more of a problem in the } \\
\text { private sector. Work harassment alone } \\
\text { explains a third of private sector nurses' } \\
\text { turnover intentions, while in the public } \\
\text { sector, three factors--work harassment, } \\
\text { management, and wellbeing--together } \\
\text { explain one-third of the variation in nurses' } \\
\text { turnover intentions. Clarify the role of } \\
\text { work harassment in private and public } \\
\text { sector nurses' turnover intentions. }\end{array}$ & $\begin{array}{l}\text { The following sentence has been added to the } \\
\text { paragraph about findings. It is: } \\
\text { "The findings suggest a differential impact of } \\
\text { management on work harassment across the } \\
\text { public and private sector." }\end{array}$ \\
\hline $\begin{array}{l}\text { At the bottom of page } 9 \text {, the authors note } \\
\text { that new nurses in Canada were already } \\
\text { experiencing work stress. This is also true } \\
\text { of beginning nurses in the UK (Mastracci } \\
\text { 2016). In the UK, a resource for new } \\
\text { nurses was the perception of ethical } \\
\text { leadership. The citation for this paper is at } \\
\text { the end of these comments. }\end{array}$ & $\begin{array}{l}\text { We thank the reviewer for this information } \\
\text { and have the reference and in-text citation to } \\
\text { the paper. Top of P10: } \\
\text { A similar situation was identified for new } \\
\text { nurses in the UK, where ethical leaders were } \\
\text { identified as a resource countering negative } \\
\text { work conditions (Mastracci, 2016). } \\
\text { Mastracci, S (2016). Beginning nurses' } \\
\text { perceptions of ethical leadership in the } \\
\text { shadow of Mid Staffs. Public Integrity, } \\
\text { 19(3): } 250-264 \text {. }\end{array}$ \\
\hline $\begin{array}{l}\text { At the top of page 17, the authors discuss } \\
\text { common source bias. The authors should } \\
\text { also incorporate the argument and findings } \\
\text { of George and Pandey, } 2017\end{array}$ & $\begin{array}{l}\text { We thank the reviewer for this information } \\
\text { and have the reference and in-text citation to } \\
\text { the paper. See p22 } \\
\text { "However, as mentioned, a common latent } \\
\text { factor was included, and several items that } \\
\text { were explained by common method variance } \\
\text { were controlled, as per George and Pandey's } \\
\text { recommendations (2017)". } \\
\text { The following reference has bene added to } \\
\text { the references. } \\
\text { George, B., \& Pandey, S. (2017). We know } \\
\text { the Yin, but where is the Yang? Toward a } \\
\text { balanced approach on common source bias in } \\
\text { public administration scholarship. Review of } \\
\text { Public Personnel Administration, 37(2), 245- } \\
270 \text {. }\end{array}$ \\
\hline
\end{tabular}




\begin{tabular}{|c|c|}
\hline $\begin{array}{l}\text { At the top of page 14, the authors note that } \\
\text { work stress is worse for public-sector } \\
\text { nurses, but again, this seems to contradict } \\
\text { what is discussed in the Abstract under } \\
\text { Findings. Please clarify. }\end{array}$ & $\begin{array}{l}\text { The following has bene added to p15 } \\
\text { On the other hand, Farr-Wharton et al (2019) } \\
\text { found work harassment was also prevalent } \\
\text { for UK and US private sector nurses. } \\
\text { On p21, the following has been added } \\
\text { "This contrasts research by Farr-Wharton et } \\
\text { al (2019) about US and UK private sector } \\
\text { nurses and suggests that the manager in } \\
\text { Bangladesh may not have the power to affect } \\
\text { how work harassment influences turnover } \\
\text { intentions for private sector nurses. Hence } \\
\text { these research findings differ from those in } \\
\text { the Anglo-American and/or European } \\
\text { countries which may suggest a significantly } \\
\text { different work context for these private sector } \\
\text { nurses working in one of the least developed } \\
\text { countries in the world". }\end{array}$ \\
\hline $\begin{array}{l}\text { Minor edits are as follows: } \\
\text { Page 1, line 29: Change nurse's to nurses' } \\
\text { Page 8, lines } 3 \text { and 5: change employee to } \\
\text { employees and change develop to } \\
\text { development } \\
\text { Page 8, line 26: Change their to there } \\
\text { Page 8, line 51: Change get to gets }\end{array}$ & These edits have been undertaken \\
\hline & Z \\
\hline
\end{tabular}

